\title{
The Existence of Dendritic Fronts
}

\author{
P. Collet ${ }^{1}$ and J.-P. Eckmann ${ }^{2}$
}

1 Laboratoire de Physique Théorique Ecole Polytechnique, F-91128 Palaiseau, Cedex, France

2 Département de Physique Théorique Université de Genève, CH-1211 Genève, Switzerland

\begin{abstract}
In this paper, we study a fourth order semilinear parabolic equation on the infinite real line. We show that in a certain parameter range, this equation has propagating front solutions (solutions tending to 0 at $+\infty$ and advancing to the right with a speed $c$ ) which leave behind them a periodic pattern in the laboratory frame. This is thus an example of spontaneous pattern formation.
\end{abstract}

\section{Table of Contents}

1. Introduction . . . . . . . . . . . . . . . . . . . . . 39

2. The Equation for the Front. Statement of the Main Theorem . . . . . . 41

3. The Existence of Stationary Solutions . . . . . . . . . . . . . 42

4. The Equation for the Front as a Fixed Point Problem . . . . . . . . 44

5. Properties of the Amplitude Equation . . . . . . . . . . . . 47

6. The Space $\mathrm{H}_{\alpha, X} \ldots \ldots \ldots \ldots \ldots \ldots \ldots \ldots \ldots \ldots \ldots$

7. The Spectrum of the Linear Problem . . . . . . . . . . . . . 55

8. A Stable Manifold Theorem for Maps with Unbounded Linear Part. . 62

9. Properties of the Linear Operator in the Main Sector. . . . . . . . . 68

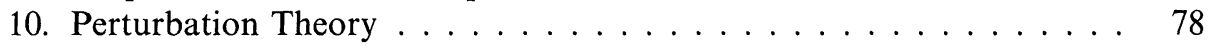

11. The Fixed Point Problem . . . . . . . . . . . . . . . . . 79

12. Bounds on the Approximate Solution . . . . . . . . . . . . . 84

13. Bounds on the Tangent Map . . . . . . . . . . . . . . . . 89

\section{Introduction}

In this paper, we discuss the existence problem for a certain type of parabolic equation motivated by the physical problem of dendrite formation. It has been pointed out (for several years, by now) that some of the parabolic (integro-) differential equations which are considered in connection with solidification and dendrite formation show, at least in numerical, and also in some physical experiments a very intriguing behaviour. One observes, in general, a one-parameter family of propagating fronts, and it seems that "most" initial data converge to a particular front, thereby leading to a selection of the propagation speed. It is furthermore conjectured that this selected speed coincides with that speed for which 
the travelling front happens to be marginally stable under linear perturbations. This subject, and the corresponding conjecture, have been studied by many authors. In particular, J. Langer has given a careful analysis of the situation. See [2] for a review and Langer and Müller-Krumbhaar [4] for a detailed analysis of a more realistic equation.

The conjectures mentioned above have been corroborated in a beautiful, and totally independent analysis of a related problem by Aronson and Weinberger [1]. See also the work by Bramson [7]. They consider equations of the form

$$
\partial_{t} U(x, t)=\partial_{x}^{2} U(x, t)+f(U(x, t)),
$$

where

$$
f(0)=0, f(1)=0, f^{\prime}(0)>0, f(U)>0 \text { for } U \in(0,1) .
$$

(The standard example of such a function is $f(U)=U-U^{3}$.) For these equations, Aronson and Weinberger prove the existence of fronts and locate the marginal speed. They further show the strong result that all positive initial conditions $U_{0}(x)$ with compact support (which do not die out as time advances) will eventually form a front which moves with the marginal speed. Their techniques of proof rely on a clever use of the maximum principle for the positive solutions of (1.1).

In dendrite formation, one observes a formation of sidebranches, i.e., of "modulations" of the shape of the tip of the dendrite as one moves back from the tip. In this paper, we begin the study of a simple equation for which the formation of fronts with modulated bulk behind the front has been conjectured. Thus these fronts advance at some constant speed, and leave in the laboratory frame a pattern which, as we shall see, resembles a sinusoidal function. In marked contrast, Eqs. (1.1) cannot lead to such fronts, since they only form fronts which are constant in the bulk. It should also be clear that the maximum principle cannot be applied because the solution will turn out not to be positive in the bulk, and because the equation is fourth order.

The simplest equation with a modulated front seems to be the complex amplitude equation

$$
\partial_{t} U=\partial_{x}^{2} U+U-U|U|^{2},
$$

which can be shown to form "spirals." However, it seems that initial conditions with compact support will all tend to the fixed phase solution, which is not modulated. It seems to us that the Eq. (1.2) presents a somewhat less interesting problem than the equation to be discussed below.

The equation we study here is

$$
\partial_{t} U(x, t)=\left(\varepsilon-\left(1+\partial_{x}^{2}\right)^{2}\right) U(x, t)-U(x, t)^{3},
$$

with $U \in \mathbb{R}$. This equation has been analyzed by Dee and Langer [3]. In the present paper, we prove that for sufficiently small but positive $\varepsilon$ Eq. (1.3) has a two-parameter family of fronts, parametrized by the speed $c(>4)$ of the front and the periodicity $\omega$ (or the amplitude) of the pattern which is formed in the bulk. For convenience, we restrict attention to the case $c-4=\mathbf{O}\left(\varepsilon^{1 / 2}\right)$ and $1-\omega=\mathbf{O}(\varepsilon)$. The estimates for the general case would not be very different, and in fact somewhat easier, since the 
parameter range we consider includes the marginally stable case, whereas in the other cases there seems to be no marginality present. In this paper, we prove the existence of fronts for Eq. (1.3). Work on the linear stability analysis of these fronts is in progress.

\section{The Equation for the Front. Statement of the Main Theorem}

Dee and Langer [3] consider the equation

$$
\partial_{t} U(x, t)=\left(\varepsilon-\left(1+\partial_{x}^{2}\right)^{2}\right) U(x, t)-U(x, t)^{3},
$$

with $U \in \mathbb{R}$. We prefer to perform a trivial scaling and to consider the equation in the form

$$
\partial_{t} U(x, t)=\left(\varepsilon-\left(1+\partial_{x}^{2}\right)^{2}\right) U(x, t)-\varepsilon U(x, t)^{3} .
$$

The stationary solutions to Eq. (2.2) are those which do not depend on the time $t$. We shall call them $S(x)$, and in fact, they will depend, in addition to $\varepsilon$ on a second parameter, $\omega$, which will be the wavelength of their period. We shall not write this $\varepsilon$ and $\omega$ dependence explicitly. A front is a solution $U$ of Eq. (2.2) which satisfies the following special conditions: Define

$$
U(x, t)=W\left(x, \eta x-c \eta^{2} t\right)
$$

where $\varepsilon=\eta^{2}$, and assume $U(x, t)$ solves Eq. (2.2). Decompose $W\left(x_{1}, x_{2}\right)$ as

$$
W\left(x_{1}, x_{2}\right)=\sum_{n \in \mathbb{Z}} e^{i n \omega x_{1}} W_{n}\left(x_{2}\right)
$$

and decompose similarly the stationary solution

$$
S\left(x_{1}\right)=\sum_{n \in \mathbb{Z}} e^{i n \omega x_{1}} S_{n}
$$

We say that $W$ is a front asymptotic to $S$ and moving with speed $\eta c$ if

$$
\lim _{x_{2} \rightarrow \infty} W_{n}\left(x_{2}\right)=0
$$

and

$$
\lim _{x_{2} \rightarrow-\infty} W_{n}\left(x_{2}\right)=S_{n}
$$

for all $n \in \mathbb{Z}$. This means that in a frame moving with speed $\eta c$, one sees at $+\infty$ the zero solution of Eq. (2.2), while at $-\infty$ (i.e. in the variable $x_{1}$ !) one sees one of the stationary solutions. Note that this also means that in the laboratory frame, the front advances with speed $\eta c$, leaving behind a pattern which does not move.

How do these fronts look? For small $\varepsilon$, and in the case $\omega=1$, the fronts have essentially the form

$$
W\left(x_{1}, x_{2}\right)=\frac{1}{3^{1 / 2}}\left(l\left(x_{2}\right) e^{i x_{1}}+l\left(x_{2}\right) e^{-i x_{1}}\right)
$$


where $l$ is the real solution of the amplitude equation

$$
4 l^{\prime \prime}+c l^{\prime}+l-l^{3}=0
$$

with the conditions $l(-\infty)=1, l(+\infty)=0, l(x)>0$ for all $x$.

Our main result is the

Main Theorem. For every $K>0$, for $c>4$ sufficiently close to 4 , and for $\varepsilon>0$ sufficiently small, there exist for every $\omega$ satisfying

$$
\left|1-\omega^{2}\right| \leqslant K \varepsilon
$$

a front asymptotic to a stationary solution with wavelength $\omega$ and moving with speed $\varepsilon^{1 / 2} c$. This solution is locally unique (modulo translations and a choice of phase).

For convenience, we shall assume $c<41 / 10$, cf. Eq. (5.18).

\section{The Existence of Stationary Solutions}

Before we can attack the front problem, we need to control the stationary solutions. In this section, we prove the existence of stationary solutions, and we bound them appropriately. Setting the time derivative equal to zero, we see from (2.2) that the stationary wave equation takes the form

$$
\varepsilon S-\left(1+\partial_{x}^{2}\right)^{2} S-\varepsilon S^{3}=0
$$

We look for real solutions of the form

$$
S(x)=\sum_{n \in \mathbb{Z}} S_{n} e^{i n \omega x}, \text { with } S_{-n}=\bar{S}_{n} \in \mathbb{C},
$$

and $S_{n}=0$ for even $n$. We may, and shall, impose in addition $S_{1}=S_{-1} \in \mathbb{R}$, thus breaking the translation invariance of Eq. (3.1). This will also imply $S_{-n}=\bar{S}_{n} \in \mathbb{R}$. A very good approximation to $S(x)$ is given by

$$
\stackrel{\&}{S}(x)=\Gamma \cos (\omega x)
$$

where $\Gamma$ is the positive root of

$$
\left(1-\omega^{2}\right)^{2}=\varepsilon\left(1-3 \Gamma^{2} / 4\right) .
$$

This choice of $\Gamma$ solves the projection of Eq. (3.1) onto $\cos \omega x$, (see below). We fix a $K>0$, and we shall study the existence problem for stationary solutions and for fronts only for $\omega$ 's satisfying

$$
0 \leqq\left(1-\omega^{2}\right)^{2} \leqq K \varepsilon^{2}
$$

We expect the marginally stable fronts to lie in this parameter range. The restriction of (3.5) to a small parameter range makes some of the estimates simpler, but seems not essential. We now write $S(x)=S(x)+s(x)$, and we view $s$ as the unknown for which we solve the existence problem. The equation for $s$ is

$$
\varepsilon s-\left(1+\partial_{x}^{2}\right)^{2} s-3 \varepsilon S^{2} s-3 \varepsilon S s^{2}-\varepsilon s^{3}-\frac{\varepsilon \Gamma^{3}}{4} \cos 3 \omega x=0
$$


since

$$
\varepsilon \dot{S}-\varepsilon S^{3}-\left(1-\omega^{2}\right)^{2} \dot{S}=\Gamma\left(\varepsilon-\frac{3 \varepsilon \Gamma^{2}}{4}-\left(1-\omega^{2}\right)^{2}\right) \cos \omega x-\frac{\varepsilon \Gamma^{3}}{4} \cos 3 \omega x .
$$

We decompose $s$ as

$$
s(x)=\sum_{n \in \mathbb{Z}} s_{n} e^{i n \omega x}, \quad \text { with } s_{-n}=\bar{s}_{n} \in \mathbb{C}, s_{1} \in \mathbb{R} .
$$

Obviously, $s_{n}=S_{n}$ for $n \neq \pm 1$, and $s_{1}=S_{1}-\Gamma / 2$. We obtain the following equations, for $n \in \mathbb{Z}$,

$$
\left(\varepsilon-\left(1-n^{2} \omega^{2}\right)^{2}\right) s_{n}-\frac{3 \varepsilon \Gamma^{2}}{4}\left(s_{n-2}+2 s_{n}+s_{n+2}\right)-\varepsilon R_{n}(s, \Gamma)=0,
$$

with

$$
\begin{aligned}
R_{n}(s, \Gamma)= & \frac{3 \Gamma}{2}\left(\sum_{p+q=n-1} s_{p} s_{q}+\sum_{p+q=n+1} s_{p} s_{q}\right) \\
& +\sum_{p+q+r=n} s_{p} s_{q} s_{r}+\frac{\Gamma^{3}}{8}\left(\delta_{n, 3}+\delta_{n,-3}\right) .
\end{aligned}
$$

We rewrite this system in the following form. For $n=1$, using $s_{1}=s_{-1}$ and Eq. (3.4), we have

$$
s_{1}=-\frac{2}{3 \Gamma^{2}}\left(\frac{3 \Gamma^{2}}{4} s_{3}+R_{1}(s, \Gamma)\right) \equiv G_{1}(s) .
$$

The cases $n \neq \pm 1$ lead to equations of the form

$$
s_{n}=-\frac{\varepsilon}{\left(1-n^{2} \omega^{2}\right)^{2}-\varepsilon+3 \varepsilon \Gamma^{2} / 2}\left(\frac{3 \Gamma^{2}}{4}\left(s_{n-2}+s_{n+2}\right)+R_{n}(s, \Gamma)\right) \equiv G_{n}(s) .
$$

Choose now any $\rho>0$ and define

$$
\|s\|=\sum_{-\infty}^{\infty} \varepsilon^{-\rho|n|}\left|s_{n}\right| .
$$

We denote by $\mathbf{B}_{\rho}$ the space of sequences $\left\{s_{n}, n \in \mathbb{Z}\right\}$, equipped with the above norm, and we want to view Eqs. (3.10), (3.11) as fixed point problem in this space. The terms occurring in $R_{n}$ can then be bounded as follows: As an example,

$$
\begin{gathered}
\sum_{n \in \mathbb{Z}} \varepsilon^{-\rho|n|}\left|\sum_{p+q=n} s_{p} s_{q}\right| \leqq \sum_{p, q \in \mathbb{Z}} \varepsilon^{-\rho|p+q|}\left|s_{p} s_{q}\right| \\
\leqq \sum_{p, q \in \mathbb{Z}} \varepsilon^{-\rho|p|}\left|s_{p}\right| \varepsilon^{-\rho|q|}\left|S_{q}\right|=\|S\|^{2} .
\end{gathered}
$$

Also, we have the inequalities

$$
\sum_{n \in \mathbb{Z}} \varepsilon^{-\rho|n|}\left|S_{n+k}\right| \leqq \varepsilon^{-\rho|k|} \sum_{n \in \mathbb{Z}} \varepsilon^{-\rho|n|}\left|S_{n}\right|
$$

and

$$
\left|s_{k}\right| \leqq \varepsilon^{-\rho|k|}\|s\|
$$


Combining these estimates, we get

$$
\left|G_{1}(s)\right| \leqq \frac{1}{2}\left|s_{3}\right|+\frac{1+\varepsilon^{2 \rho}}{\Gamma}\|s\|^{2}+\frac{2 \varepsilon^{\rho}}{3 \Gamma^{2}}\|s\|^{3},
$$

and hence,

$$
\varepsilon^{-\rho}\left|G_{1}(s)\right| \leqq \frac{\varepsilon^{2 \rho}}{2}\|s\|+\frac{\varepsilon^{-\rho}+\varepsilon^{\rho}}{\Gamma}\|s\|^{2}+\frac{2}{3 \Gamma^{2}}\|s\|^{3} .
$$

Since $G_{1}$ is multilinear, it is easy to calculate and estimate the tangent map, $D G_{1}$, and one gets

$$
\varepsilon^{-\rho}\left|D G_{1}(s) w\right| \leqq\left(\frac{\varepsilon^{2 \rho}}{2}+2 \frac{\varepsilon^{-\rho}+\varepsilon^{\rho}}{\Gamma}\|s\|+3\|s\|^{2}\right)\|w\| .
$$

For the cases $n \neq \pm 1$, we have similar equations

$$
\sum_{n \neq \pm 1} \varepsilon^{\rho|n|}\left|G_{n}(s)\right| \leqq \mathbf{O}(\varepsilon)\left(\left(\varepsilon^{2 \rho}+\varepsilon^{-2 \rho}\right)\|s\|+\varepsilon^{-\rho}\|s\|^{2}+\|s\|^{3}+\varepsilon^{-3 \rho}\right)
$$

and

$$
\sum_{n \neq \pm 1} \varepsilon^{-\rho|n|}\left|D G_{n}(s) w\right| \leqq \mathbf{O}(\varepsilon)\left(\varepsilon^{-2 \rho}+\varepsilon^{-\rho}\|s\|+\|s\|^{2}\right)\|w\| .
$$

We now restrict $\rho$ to the interval $0<\rho<1 / 4$, and we choose $\tau$ satisfying $1-3 \rho$ $>\tau>\rho$. It is easy to check from (3.17) and (3.19) that for sufficiently small $\varepsilon$ one has

$$
\|G(s)\|<\varepsilon^{\tau}, \quad \text { if }\|s\|<\varepsilon^{\tau},
$$

where $G=\left\{G_{n}\right\}_{n \in \mathbb{Z}}$. Similarly, one finds from (3.18) and (3.20)

$$
\|D G(s)\| \leqq \mathbf{O}\left(\varepsilon^{\mu}\right), \quad \text { if }\|s\|<\varepsilon^{\tau},
$$

with $\mu=\min (\tau-\rho, 2 \rho)$. We apply the contraction mapping principle to the equation $G(s)=s$, and the above estimates imply the

Proposition 3.1. Let $1-3 \rho>\tau>\rho>0$. For sufficiently small $\varepsilon$ there is, for every $\omega$ satisfying Eq. (3.5), a solution to Eq. (3.1). It is of the form

$$
S(x)=\Gamma \cos \omega x+s(x),
$$

with $s(x)=\sum_{n} s_{n} e^{i n \omega x},\left\{s_{n}\right\}_{n \in \mathbb{Z}} \in \mathbf{B}_{\rho}$, and $\|s\|<\varepsilon^{\tau}$. Such solutions are unique.

Remark. One has the bounds which are better than those of the proposition:

$$
\left|s_{1}\right|,\left|s_{-1}\right|<\varepsilon^{4 \rho} \text {. }
$$

Proof. Use the identities (3.9), (3.10), and $\tau>\rho$.

\section{The Equation for the Front as a Fixed Point Problem}

We begin with a formulation of the front problem. We want to view the front as the perturbation of a periodically modulated amplitude equation. This is done in the present section. We formulate the problem as a system of differential equations in these perturbations. In later sections, we shall give the definitions of adequate 
function spaces in which these equations will be seen to have solutions. The existence of these solutions will follow from an application of the contraction mapping principle.

We now consider functions $W$ of the form

solving the equation

$$
W\left(x, \eta x-c \eta^{2} t\right)
$$

$$
\partial_{t} W=-\left(1+\partial_{x}^{2}\right)^{2} W+\varepsilon W-\varepsilon W^{3},
$$

where $\varepsilon=\eta^{2}$. We decompose $W$ as

$$
W\left(x_{1}, x_{2}\right)=\sum_{n \in \mathbb{Z}} e^{i n \omega x_{1}} W_{n}\left(x_{2}\right),
$$

and we look for a solution satisfying

$$
W_{n}(+\infty)=0, \quad W_{n}(-\infty)=S_{n},
$$

where $S_{n}$ is the component of the stationary solution, with the corresponding $\omega$, discussed in Sect. 3. We shall also require $W_{-n}=\bar{W}_{n}$, i.e., we look for real functions $W$. Since $S_{n}=0$ for even $n$, we require also $W_{n}=0$ for even $n$.

Since we have $x_{1}=x$ and $x_{2}=\eta x-c \eta^{2} t$, we see that $W$ solves

$$
-\eta^{2} c \partial_{2} W=\eta^{2} W-\left(1+\partial_{1}^{2}+2 \eta \partial_{1} \partial_{2}+\eta^{2} \partial_{2}^{2}\right)^{2} W-\eta^{2} W^{3},
$$

where $\partial_{i}=\partial / \partial_{x_{i}}$, for $i=1,2$. The equation for $W_{n}$ is then

$$
\eta^{2} W_{n}-\left(1-n^{2} \omega^{2}+2 i \eta n \omega \partial_{2}+\eta^{2} \partial_{2}^{2}\right)^{2} W_{n}+\eta^{2} c \partial_{2} W_{n}=\sum_{p+q+r=n} W_{p} W_{q} W_{r} .
$$

We shall use, throughout, the notation

$$
\mu=\omega n \text {. }
$$

It is now natural to consider the one parameter family of linear operators $A_{\mu}$ defined by

$$
A_{\mu} W(x)=\eta^{2} W(x)-\left(\left(1-\mu^{2}\right)+2 i \eta \mu \partial_{x}+\eta^{2} \partial_{x}^{2}\right)^{2} W(x)+\eta^{2} c \partial_{x} W(x) .
$$

Upon expansion, we find

$$
\begin{aligned}
A_{\mu}= & -\varepsilon^{2} \partial_{x}^{4}-4 i \varepsilon^{3 / 2} \mu \partial_{x}^{3}-\varepsilon\left(2\left(1-\mu^{2}\right)-4 \mu^{2}\right) \partial_{x}^{2} \\
& +\left(\varepsilon c-4 i \varepsilon^{1 / 2} \mu\left(1-\mu^{2}\right)\right) \partial_{x}+\left(\varepsilon-\left(1-\mu^{2}\right)^{2}\right) \mathrm{id} .
\end{aligned}
$$

We want to view the system of Eqs. (4.6) as a fixed point problem. We can rewrite (4.6) as

$$
A_{\omega n} W_{n}=\eta^{2} \sum_{p+q+r=n} W_{p} W_{q} W_{r} .
$$

We guess an approximate solution and transform the problem somewhat. We shall call main sector the sector $n= \pm 1$. Note that the equation for $n=1$ is the complex conjugate of the one for $n=-1$. In the main sector, we make an ansatz of the form

$$
W_{1}(x)=S_{1} w_{1}(x), \quad w_{1}(x)=l(x)+a(x)+i b(x),
$$


where $a$ and $b$ are real functions. The function $l$ solves the amplitude equation

$$
4 l^{\prime \prime}(x)+c l^{\prime}(x)+l(x)-l^{3}(x)=0
$$

with the conditions $l(x) \geqq 0, l(-\infty)=1, l(\infty)=0$. We also break translation invariance by requiring $l(0)=1 / 2$. With this choice of $l$, it will be seen that $a$ and $b$ are small, in an appropriate function space.

In the sectors $n \neq \pm 1$, we set

$$
W_{n}(x)=\stackrel{\circ}{W}_{n}(x)+w_{n}(x), \quad \text { for } n \neq \pm 1,
$$

with

$$
\stackrel{\circ}{W}_{n}(x)=S_{n} l(x) \text {. }
$$

Here, the choice of the function $l$ as an amplitude is somewhat arbitrary. In fact, any "nice" function with value 1 at $-\infty$ and value 0 at $+\infty$ would do. But we stress again that in the main sector, the choice of $l$ is crucial for what follows.

We denote $\mathbf{w}=\left\{w_{n}\right\}_{n \in \mathbb{Z}}$. In the sectors $n \neq \pm 1$, the equation takes the form

$$
A_{\omega n} w_{n}=\eta^{2} \sum_{p+q+r=n} W_{p} W_{q} W_{r}-S_{n} A_{\omega n} l(x)=: h_{n}(\mathbf{w}) .
$$

The interesting sector is the main sector where all terms will be seen to be at least of order $\eta^{2}$. We first define $\sum^{*}$ as the sum over the set of $p, q, r$ for which

$$
p+q+r=1, \quad|p|+|q|+|r| \neq 3 .
$$

We note for later use that this implies, because $p, q, r$ are odd, that

$$
|p|+|q|+|r| \geqq 5 \text {. }
$$

Now we write the equation in the main sector as

$$
S_{1} A_{\omega} w_{1}-3 S_{1}^{3} \eta^{2} w_{1}\left|w_{1}\right|^{2}=\eta^{2} \sum^{*} W_{p} W_{q} W_{r}
$$

We next define

$$
B_{\omega}=\eta^{-2} A_{\omega} .
$$

Note that $A_{\omega}$ is of order $\omega-1$. Because $\omega-1=\mathbf{O}\left(\eta^{2}\right)$, seemingly singular terms in $B_{\omega}$ will stay bounded as $\eta \rightarrow 0$. We will prove this fact in detail later. Our final form for the equation in the main sector is now

$$
B_{\omega} w_{1}-w_{1}\left|w_{1}\right|^{2}=\frac{1}{S_{1}}\left(\sum^{*} W_{p} W_{q} W_{r}-w_{1}\left|w_{1}\right|^{2}\left(1-3 S_{1}^{2}\right)\right)=: h_{1}(\mathbf{w}) .
$$

This equation will be analyzed in Sect. 9. Our strategy will be to study in detail the operator $B_{1}$ and to view $B_{1}-B_{\omega}$ as a perturbation. This perturbation will be small for small $\eta$ since we are assuming $1-\omega=\mathbf{O}\left(\eta^{2}\right)$.

Note that by the definitions in Sect. $3,3 S_{1}^{2}$ is close to 1 . Furthermore, since $S_{n}$ is small for $n \neq \pm 1$ the cubic term in $h_{1}$ is also small. In Sect. 9, we shall essentially invert the operator

$$
L: w \rightarrow B_{1} w-w|w|^{2}
$$


and write (4.19) as

$$
w_{1}=L^{-1} h_{1}(\mathbf{w}) \text {. }
$$

In fact, we shall first perform a translation in function space, by $l$ as in (4.11), and then invert the operator acting on the translated variable. Thus, Eq. (4.19) leads to a fixed point equation with a small right-hand side. We also invert the $A_{\omega n}$ (in Sect. 7) and write (4.15) as

$$
w_{n}=A_{\omega n}^{-1} h_{n}(\mathbf{w}) .
$$

Although the sum defining $h_{n}$ is not restricted, there is a factor $\eta^{2}$ which renders the right-hand side of (4.21) small. Thus it should be intuitively clear that the existence problem for the propagating wave has been reduced to a fixed point problem with small right-hand side, so that the contraction mapping principle can be applied.

\section{Properties of the Amplitude Equation}

In the following discussion, we fix $c>4$, and we consider the equation

$$
4 l^{\prime \prime}+c l^{\prime}+l\left(1-l^{2}\right)=0
$$

We are only interested in solutions $l$ which satisfy

$$
\begin{aligned}
0 & \leqq l(x) \leqq 1, \\
l(-\infty) & =1, \quad l(+\infty)=0,
\end{aligned}
$$

and to break translation invariance, we shall require

$$
l(0)=\frac{1}{2}
$$

This problem has been extensively discussed in Aronson and Weinberger [1], and we rely on some of their results. However, we shall need some additional estimates which we derive shortly. It is known that the solutions to (5.1)-(5.4) satisfy

$$
l^{\prime}(x)<0, \quad \text { for all } x \in \mathbb{R} .
$$

We want to discuss in detail the decay of the solution near $+\infty$ and its approach to 1 near $-\infty$. To do this, we need first an a priori bound on the derivative of $l$. The minimum of $l^{\prime}$ (i.e. the maximum of $\left|l^{\prime}\right|$ ) is attained when $l^{\prime \prime}=0$. Hence we have, for all $x \in \mathbb{R}$,

$$
l^{\prime}(x) \geqq-\frac{1}{c_{z \in[0,1]}} \sup _{z} z\left(1-z^{2}\right) \geqq-A,
$$

where $A=2 /\left(3^{3 / 2} c\right)$. We also need an upper bound on $l^{\prime}$ (i.e. a lower bound on $\left.\left|l^{\prime}\right|\right)$. Since $-1 \leqq l^{3}-l \leqq 0$ and $l^{\prime}<0$, we have

$$
-1 \leqq-l+l^{3}-c l^{\prime} \leqq 0+c A=2 / 3^{3 / 2},
$$

and hence $4 l^{\prime \prime}=-l+l^{3}-c l^{\prime}$ implies

$$
\left|l^{\prime \prime}\right| \leqq \frac{1}{4}
$$


It follows that for every $x, h \in \mathbb{R}$, one has

$$
\left|l^{\prime}(x+h)-l^{\prime}(x)\right| \leqq h / 4 .
$$

Therefore, there is a function $\sigma_{1}(x, h)$ satisfying $\left|\sigma_{1}(x, h)\right| \leqq c / 4$, such that

$$
\begin{aligned}
4 l^{\prime \prime}(x+h) & =-c l^{\prime}(x+h)-l(x+h)\left(1-l(x+h)^{2}\right) \\
& =-c l^{\prime}(x)+h \sigma_{1}(x, h)-l(x)+l^{3}(x)-\int_{x}^{x+h} d s l^{\prime}(s)\left(1-3 l(s)^{2}\right) \\
& =-c l^{\prime}(x)-l(x)+l^{3}(x)+h \sigma_{2}(x, h) .
\end{aligned}
$$

Here, $\left|\sigma_{2}(x, h)\right| \leqq c / 4+2 A \leqq c / 2$. Integrating again, with respect to $h$, we get

$$
4 l^{\prime}(x+h)=4 l^{\prime}(x)+h\left(l^{3}(x)-l(x)-c l^{\prime}(x)\right)+h^{2} \sigma_{3}(x, h)
$$

with a bound $\left|\sigma_{3}(x, h)\right| \leqq c / 4$.

We now fix an $x \in \mathbb{R}$ and we denote $\rho=l(x)$, and $\sigma=\rho-\rho^{3}$. Note that $\sigma$ is positive. We assume now that

$$
\left|l^{\prime}(x)\right|<\min \left(\frac{\sigma}{2 c}, \frac{\sigma^{2}}{16 c}\right) .
$$

(Since $\sigma<1$ and $c>1$ the second term of the min is the smaller.) We shall show that (5.10) leads to a contradiction. In fact, combining (5.10) with the definition of $\sigma$, we get

$$
\rho^{3}-\rho-c l^{\prime}(x) \leqq-\frac{\sigma}{2}
$$

so that for $h_{0}=\sigma / c$ we find

$$
l(x)^{3}-l(x)-c l^{\prime}(x)-h_{0} \sigma_{3}\left(x,-h_{0}\right) \leqq-\frac{\sigma}{4} .
$$

Combining (5.9), (5.10) and (5.11), we see that

$$
4 l^{\prime}\left(x-h_{0}\right)>4 l^{\prime}(x)+h_{0} \frac{\sigma}{4}>0,
$$

a contradiction. Hence we have shown the

Lemma 5.1. There is a positive function $F(\rho, c)$, such that

$$
-A=-2 /\left(3^{3 / 2} c\right) \leqq l^{\prime}(x) \leqq-F(l(x), c) .
$$

One has

$$
F(\rho, c)=\left(\frac{\rho-\rho^{3}}{2 c}, \frac{\left(\rho-\rho^{3}\right)^{2}}{16 c}\right) .
$$

We next give an upper bound on $l$ at $+\infty$. From (5.1) and (5.4), we have, for $x \geqq 0$,

$$
4 l^{\prime \prime}(x)+c l^{\prime}(x)+\frac{3}{4} l(x)=l(x)\left(l(x)^{2}-\frac{1}{4}\right) \leqq 0 .
$$


We now consider the roots

$$
\delta_{ \pm}=\frac{-c \pm\left(c^{2}-12\right)^{1 / 2}}{8}
$$

of the polynominal $4 z^{2}+c z+3 / 4$. By the maximum principle (see Aronson and Weinberger [1]), the inequality (5.13) implies that $l$ is bounded above by a solution $v$ of the equation $4 v^{\prime \prime}+c v^{\prime}+3 v / 4=0$. Clearly

$$
v(x)=\alpha_{+} e^{\delta_{+} x}+\alpha_{-} e^{\delta_{-} x},
$$

with

$$
\begin{aligned}
& \alpha_{+}+\alpha_{-}=l(0)=1 / 2, \\
& \alpha_{+} \delta_{+}+\alpha_{-} \delta_{-}=l^{\prime}(0) .
\end{aligned}
$$

Therefore, $\alpha_{+}= \pm\left(l^{\prime}(0)-\delta_{\mp} / 2\right) /\left(\delta_{+}-\delta_{-}\right)$. By (5.6), we see that there is a constant $C_{1}$ such that uniformly in $c \geqq 4$, we have $\left|\alpha_{ \pm}\right| \leqq C_{1} / 2$. Since $0>\delta_{+}>\delta_{-}$, we find

$$
l(x) \leqq C_{1} e^{\delta_{+} x}, \quad \text { as } x \rightarrow+\infty .
$$

We next improve this a priori estimate. Equation (5.1) and inequality (5.15) imply

$$
4 l^{\prime \prime}(x)+c l^{\prime}(x)+l(x)=l^{3}(x) \leqq C_{1}^{3} e^{3 \delta_{+} x} .
$$

For $c=4$ we have $\delta_{+}=-1 / 4$, so that for $c$ sufficiently close to 4 we find $\left|3 \delta_{+}\right|>5 / 8$. We consider now the equation

$$
4 w^{\prime \prime}+c w^{\prime}+w=C_{1}^{3} e^{-5 x / 8} .
$$

Since $e^{-5 x / 8} \geqq e^{3 \delta_{+} x}$ for $x \geqq 0$, the solution of Eq. (5.17) will be an upper bound on $l$ (for $x \geqq 0$ ), provided $w(0)=l(0), w^{\prime}(0)=l^{\prime}(0)$. We define $\gamma+$ and $\gamma-$ as the roots of the polynomial $4 z^{2}+c z+1$, i.e.,

$$
\gamma_{ \pm}=\frac{-c \pm\left(c^{2}-16\right)^{1 / 2}}{8}
$$

Then we get the solution, valid for $4<c<41 / 10$,

$$
w(x)=C_{2}(c) e^{y+x}+C_{3}(c) e^{\gamma-x}+C_{4}(c) e^{-5 x / 8},
$$

with

$$
\begin{aligned}
C_{4}(c) & =C_{1}^{3} /(41 / 16-5 c / 8), \\
C_{2}(c)+C_{3}(c) & =l(0)-C_{4}(c), \\
C_{2}(c) \gamma_{+}+C_{3}(c) \gamma_{-} & =l^{\prime}(0)+5 C_{4}(c) / 8 .
\end{aligned}
$$

Clearly, one can find solutions to (5.20). Note, however, that they diverge as $c \rightarrow 4$.

For $x \geqq 0,(c-4)^{1 / 2} x<1$, it will still be possible to bound the quantity

$$
w(x) \frac{e^{x / 2}}{1+x}
$$


which in turn leads to a bound for $l$. Observe first that

$$
\gamma_{+}+1 / 2 \leqq \mathbf{O}\left((c-4)^{1 / 2}\right)
$$

as long as $c$ is bounded (which we assume). We can now write, omitting the $c$ dependence of the $C_{j}$,

$$
C_{2} e^{y_{+} x}+C_{3} e^{y-x}=\left(C_{2}+C_{3}\right) e^{y_{+} x}+C_{3} e^{y_{+} x}\left(e^{\left(y_{-}-y_{+}\right) x}-1\right) .
$$

By using Taylor's formula to second order, and denoting $\delta=(c-4)^{1 / 2}$, we see that this leads to a bound

$$
\left|w(x) \frac{e^{x / 2}}{1+x}\right| \leqq \mathbf{O}(1) e^{\mathbf{O}(\delta x)}+\mathbf{O}\left(\frac{1}{\delta}\right) \frac{e^{\mathbf{O}(\delta x)} \delta x}{1+x}+\mathbf{O}\left(\frac{1}{\delta}\right) \frac{e^{\mathbf{O}(\delta x)} \delta^{2} x^{2}}{1+x}
$$

This implies the required bound and hence the

Lemma 5.2. For $0 \leqq(c-4)^{1 / 2} x<1$, the solution $l(x)$ of Eqs. (5.1)-(5.4) satisfies

$$
l(x) \frac{e^{x / 2}}{1+x} \leqq \mathbf{O}(1)
$$

We next give a lower bound on $l(x)$ for $x \geqq 0$.

Lemma 5.3. With the definition (5.18) of $\gamma_{+}$, we have the bound

$$
l(x) \geqq \frac{1}{4} e^{\gamma+x} .
$$

Proof. By Lemma 5.1, we have

$$
\operatorname{cl}(0) / 8+l^{\prime}(0) \geqq \frac{1}{4}-\frac{2}{4 \cdot 3^{3 / 2}}>0 .
$$

By the maximum principle, it is again clear that $l$ is bounded below by the solution $v$ of the equation

$$
4 v^{\prime \prime}(x)+c v^{\prime}(x)+v(x)=0,
$$

with the initial conditions $v(0)=l(0), v^{\prime}(0)=l^{\prime}(0)$. We find $v(x)=\alpha_{+} e^{y_{+} x}+\alpha_{-} e^{y-x}$, where

$$
\alpha_{+}+\alpha_{-}=1 / 2, \quad \alpha_{+} \gamma_{+}+\alpha_{-} \gamma_{-}=l^{\prime}(0) .
$$

This implies $\alpha_{+}-\alpha_{-}=\left(l^{\prime}(0)+c l(0) / 8\right) / \Delta$, where $\Delta=\left(c^{2}-16\right)^{1 / 2} / 8$. By (5.26), we find $\alpha_{+}>\alpha_{-}$. We rewrite

$$
v(x)=w(x) e^{y+x}, \quad w(x)=\alpha_{+}+\alpha_{-} e^{-2 \Delta x} .
$$

Note that $w(0)=1 / 2$. Now if $\alpha_{-} \geqq 0$, then $w(x) \geqq \alpha_{+}>1 / 4$, since $\alpha_{+}+\alpha_{-}=1 / 2$ and $\alpha_{+}>\alpha_{-}$. If $\alpha_{-}<0$, then $w^{\prime}(x)=-2 \Delta \alpha_{-} e^{-2 \Delta x}>0$, so that $w(x)>w(0)=1 / 2$. The assertion follows from $l(x) \geqq v(x)=e^{\gamma+x} w(x)$.

We next deduce an upper bound on $\left|l^{\prime}(x)\right|$ for $x \geqq 0$.

Lemma 5.4. For every $c>4$, there is a constant $C_{5}(c)$, such that

$$
\left|l^{\prime}(x)\right| \leqq C_{5}(c) e^{y+x}, \text { for all } x \geqq 0 .
$$


Proof. By definition, $l$ satisfies the equation

$$
4 l^{\prime} l^{\prime \prime}+c l^{2}+l l^{\prime}\left(1-l^{2}\right)=0 .
$$

We set $v=l^{\prime 2}$, so that

$$
2 v^{\prime}+c v+l l^{\prime}\left(1-l^{2}\right)=0 .
$$

Setting next $v(x)=y(x) e^{-c x / 2}$, we get

$$
2 y^{\prime}(x)+e^{c x / 4} l(x) e^{c x / 4} l^{\prime}(x)\left(1-l^{2}(x)\right)=0 .
$$

Therefore

$$
2 y^{\prime}(x)-y^{1 / 2}(x) e^{c x / 4} l(x)\left(1-l^{2}(x)\right)=0
$$

and hence

$$
\frac{2 y^{\prime}(x)}{y^{1 / 2}(x)}=e^{c x / 4} l(x)\left(1-l^{2}(x)\right) \leqq e^{c x / 4} l(x) \leqq C(c) e^{\left(\gamma_{+}+c / 4\right) x},
$$

by (5.19). This implies

$$
4 y^{1 / 2}(x)-4 y^{1 / 2}(0) \leqq \frac{C(c)}{\Delta+c / 8}\left(e^{(\Delta+c / 8) x}-1\right),
$$

where $\Delta=\left(c^{2}-16\right)^{1 / 2} / 8$. Substituting the definition of $y$, we see that

$$
\begin{aligned}
\left|l^{\prime}(x)\right|=e^{-c x / 4} y^{1 / 2}(x) & \leqq\left|l^{\prime}(0)\right| e^{-c x / 4}+\frac{C(c)\left(e^{(\Delta-c / 8) x}-e^{-c x / 4}\right)}{4(\Delta+c / 8)} \\
& \leqq \text { const. } e^{(\Delta-c / 8) x}=\text { const. } e^{y+x} .
\end{aligned}
$$

We have used the inequality $\Delta-c / 8>-c / 4$. The assertion is proved.

The preceding argument can also be used to provide a lower bound on $\left|l^{\prime}\right|$. Starting with Eq. (5.35), we get from $l(x) \leqq 1 / 2$ the inequality

$$
\frac{2 y^{\prime}(x)}{y^{1 / 2}(x)} \geqq \frac{3}{4} e^{c x / 4} l(x) .
$$

Upon integrating as before, we get from (5.25),

$$
4 y^{1 / 2}(x)-4 y^{1 / 2}(0) \geqq \int_{0}^{x} d t \frac{3}{16} e^{(c / 4+\gamma+) t}
$$

Using again the definition of $y$, one easily reaches the following conclusion.

Lemma 5.5. The function $\left|l^{\prime}\right|$ is bounded below: There is a constant $C_{6}(c)>0$ such that for every $x \geqq 0$, one has

$$
\left|l^{\prime}(x)\right| \geqq C_{6}(c) e^{\gamma+\lambda} .
$$

We also need a bound on $l^{\prime} / l$ which is uniform in $c$. For this, we consider $y=l^{\prime} / l$. Since $y^{\prime}=l^{\prime \prime} / l-\left(l^{\prime} / l\right)^{2}$, we see that $y$ satisfies the equation

$$
4 y^{\prime}+\left(4 y^{2}+c y+1-l^{2}\right)=4 y^{\prime}+P(y, x)=0 .
$$


The roots of $P(\cdot, x)$ are at

$$
r_{ \pm}(x)=\frac{-c \pm\left(c^{2}-16\left(1-l(x)^{2}\right)\right)^{1 / 2}}{8}<0 .
$$

Assume there is an $x_{0}$ for which $y\left(x_{0}\right)<r_{-}\left(x_{0}\right)$. Then Eq. (5.41) implies

$$
y^{\prime}\left(x_{0}\right)=-P\left(y\left(x_{0}\right), x_{0}\right) / 4<0,
$$

and thus $y(x)<r_{-}\left(x_{0}\right)$ for all $x \geqq x_{0}$, since $r_{-}$is increasing. This implies

$$
y^{\prime}(x)<-P\left(y\left(x_{0}\right), x_{0}\right) / 4<0 \text { for all } x \geqq x_{0},
$$

and hence $y(x)$ diverges linearly to $-\infty$ as $x \rightarrow \infty$. For every $c>4$, this contradicts the inequalities (5.25) and (5.30). Hence, we have, for all $x \geqq 0$,

$$
\left|\frac{l^{\prime}(x)}{l(x)}\right| \leqq \frac{c+\left(c^{2}-16\right)^{1 / 2}}{8} .
$$

We can use the above argument to produce an upper bound on $l^{\prime} / l$. We observe that (5.41) implies that if $y\left(x_{0}\right)>r_{+}\left(x_{0}\right)$ for some $x_{0} \geqq 0$, then $y^{\prime}\left(x_{0}\right)<0$. Therefore, we must have, for all $x \geqq 0$,

$$
y(x) \leqq \max \left(y(0), r_{+}(x)\right) .
$$

Hence, we find, for $x \geqq 0$,

$$
\left|\frac{l^{\prime}(x)}{l(x)}\right| \geqq \min \left(\left|\frac{l^{\prime}(0)}{l(0)}\right|,\left|r_{+}(0)\right|\right) .
$$

We next bound $l$ near $-\infty$. These bounds are more straightforward than those near $+\infty$ because 1 is an unstable solution of Eq. (5.1).

Lemma 5.6. For all $c$ near 4 , the function $l(x)$ satisfies near $-\infty$ a bound of the form

$$
1-C_{8} e^{\beta x}<l(x)<1
$$

where

$$
\beta=\frac{-c+\left(c^{2}+32\right)^{1 / 2}}{8}
$$

Proof. We set $u(x)=1-l(x)$, and then we see that $u$ satisfies

$$
4 u^{\prime \prime}+c u^{\prime}-2 u+3 u^{2}-u^{3}=0 \text {. }
$$

The linear equation

$$
4 v^{\prime \prime}+c v^{\prime}-2 v=0
$$

has only one solution which decays at $-\infty$, namely

$$
v(x)=\text { const. } e^{\beta x} .
$$

Therefore, $u^{\prime} / u \rightarrow \beta$ as $x \rightarrow-\infty$, provided $u$ is bounded. In fact, $u(x)$ is the unstable 
manifold of the flow defined by the equations

$$
\begin{aligned}
& u^{\prime}=s, \\
& s^{\prime}=-\frac{c}{4} s+\frac{1}{2} u-\frac{3}{4} u^{2}+\frac{1}{4} u^{3} .
\end{aligned}
$$

The unstable direction at $(0,0)$ is $\beta u=s$, and hence by the existence of an unstable manifold, there is a $u_{0}$ such that $0<u_{0}<1 / 2$, and

$$
|s(u)-\beta u| \leqq u^{3 / 2}, \quad \text { for } 0 \leqq u \leqq u_{0} .
$$

One can find (by choosing possibly a smaller positive $u_{0}$ ) an $x_{0}$ such that $u\left(x_{0}\right)=u_{0}$. Then Eq. (5.49) implies upon integration, for $x \leqq x_{0}$,

$$
u_{0} e^{\left(\beta+u_{0}^{3 / 2}\right)\left(x-x_{0}\right)} \leqq u(x) \leqq u_{0} e^{\left(\beta-u_{0}^{3 / 2}\right)\left(x-x_{0}\right)} .
$$

By choosing again $u_{0}$ smaller if necessary, we may achieve

$$
\frac{1}{2}\left(\beta-u_{0}^{3 / 2}\right) \geqq \frac{1}{3} \beta \text {. }
$$

Substituting in Eq. (5.49), we get, for $x \leqq x_{0}$,

$$
|s(u) / u-\beta| \leqq u_{0}^{1 / 2} e^{\beta\left(x-x_{0}\right) / 3} .
$$

This inequality leads, after integration, to

$$
\left|\log u(x)-\beta\left(x-x_{0}\right)-\log u_{0}\right| \leqq u_{0}^{1 / 2} \frac{3}{\beta},
$$

so that for $x \leqq x_{0}$,

$$
u_{0} e^{-3 u_{0}^{1 / 2} / \beta} e^{\beta\left(x-x_{0}\right)} \leqq u(x) \leqq u_{0} e^{3 u_{0}^{1 / 2} / \beta} e^{\left(x-x_{0}\right)} .
$$

Thus we have shown the required bounds for $x \leqq x_{0}$.

We now extend this bound to all negative $x$. Since $l(0)=1 / 2=u(0)$, and $u^{\prime}(x)>0$, we see that for $x \in\left[x_{0}, 0\right]$ one has

$$
u\left(x_{0}\right) \leqq u(x) \leqq u(0)
$$

Thus for all $c$ near 4 , there are constants $C_{7}>0$ and $C_{8}<\infty$ such that

$$
C_{7} e^{\beta x} \leqq u(x) \leqq C_{8} e^{\beta x}
$$

The lemma is proved.

Our next bonds are on $l^{\prime}$ near $-\infty$. In this case, we differentiate Eq. (5.1), and we set $u(x)=l^{\prime}(x)$. Then the equation for $u$ is

$$
4 u^{\prime \prime}+c u^{\prime}+u\left(1-3 l^{2}\right)=0 .
$$

For $x$ near $-\infty$, the solution is governed by the equation $4 u^{\prime \prime}+c u^{\prime}-2 u=0$, which has constant coefficients and which we have already discussed above. The variation of the coefficients, due to $l$ decays faster than $e^{\beta x}$ near $-\infty$, and hence we find by methods totally analogous to the ones used above the

Lemma 5.7. For all $c$ near 4 , the function $l^{\prime}(x)$ satisfies near $-\infty$ a bound of the form

$$
0<C_{9} e^{\beta x}<-l^{\prime}(x)<C_{10} e^{\beta x},
$$


where $\beta$ was defined in Eq. (5.45).

Remark. The methods used above show that all higher derivatives of lat $+\infty$ or $-\infty$ decay at the same rate as $l$ and $l^{\prime}$, (respectively as $1-l$ and $l^{\prime}$ near $\left.-\infty\right)$.

\section{The Space $\mathbf{H}_{\alpha, X}$}

We define a weight function $m$ which will serve for our norms:

$$
m(x)=e^{-\alpha x}+\frac{e^{x / 2}}{1+|x|},
$$

where $\alpha<\beta$ and $\beta$ was defined in Eq. (5.45).

Definitions. We denote $\mathbf{H}_{a, X}$ the space of functions defined by

$$
\mathbf{H}_{\alpha, X}=\left\{f\left|f:(-\infty, X] \rightarrow \mathbb{C}, f \in \mathscr{C}^{0}((-\infty, X]), \sup _{x \in(-\infty, X]}\right| f(x) \mid m(x)<\infty\right\},
$$

and we write \|\|$_{\mathbf{H}_{\alpha, X}}$ for the corresponding norm.

Lemma 6.1. For $q \gg 1$ and $x \leqq X$ one has the bounds

$$
m(x) \int_{-\infty}^{x} d y e^{-q(x-y)} / m(y) \leqq \mathbf{O}\left(q^{-1}\right)
$$

and

$$
m(x) \int_{x}^{X} d y e^{q(x-y)} / m(y) \leqq \mathbf{O}\left(q^{-1}\right) .
$$

Proof. The proof is straightforward. We distinguish the cases $x \geqq 0$ and $x \leqq 0$ and the integrals (6.3) and (6.4). We first note the obvious bounds

$$
1 / m(y) \leqq\left\{\begin{array}{ll}
\text { const. } e^{\alpha y} & \text { if } y \leqq 0 \\
\text { const. }(1+y) e^{-y / 2} & \text { if } y \geqq 0
\end{array} .\right.
$$

Noting that $\alpha=\mathbf{O}(1)$ and $q \gg 1$, we get the asserted bounds by straightforward integration.

The following inequalities will be used in Sect. 9. Define

$$
g(x)=e^{-x} \int_{-\infty}^{x} d s f(s) e^{s}
$$

i.e. $g=\left(1+\partial_{x}\right)^{-1} f$.

Lemma 6.2. If $\|f\|_{\mathbf{H}_{\alpha, X}}$ is finite, then

$$
\|g\|_{\mathbf{H}_{\alpha, X}} \leqq \mathbf{O}(1)\|f\|_{\mathbf{H}_{\alpha, X}} .
$$

Proof. Note that $|f(s)| \leqq\|f\|_{\mathbf{H}_{\alpha, X}} \mathbf{O}(1)(1+s) e^{-s / 2}$ for $s \geqq 0$. Similarly, if $s \leqq 0$, then $f$ decays like $e^{-\alpha|s|}$. Therefore, if $x \leqq 0$, we find

$$
g(x) \leqq\|f\|_{\mathbf{H}_{\alpha, X}} \mathbf{O}(1) e^{-x} \int_{-\infty}^{x} d s e^{\alpha s+s} \leqq \mathbf{O}\left(e^{\alpha x}\right)\|f\|_{\mathbf{H}_{\alpha, X}} .
$$


On the other hand, if $x \geqq 0$, then we have

$$
g(x) \leqq \mathbf{O}\left(e^{-x / 2}\right)(1+x)\|f\|_{\mathbf{H}_{\alpha, X}} .
$$

Lemma 6.3. If $X>0$ and $q \gg 1$, then one has for all $x<X$,

$$
\frac{m(x)}{m(X)} e^{-q(X-x)} \leqq 1
$$

Proof. The proof is obvious from the definition of $m$.

\section{The Spectrum of the Linear Problem}

We give here bounds on the spectrum of $A_{\omega n}$ when $\omega$ is near 1 and $n \neq \pm 1$. Then we deal with the case $n=1$. The characteristic polynomial of $A_{\omega n}$ is

$$
P_{\mu}(q)=\eta^{2}-\left(1-(\mu+\eta q)^{2}\right)^{2}+i \eta^{2} c q,
$$

when $\mu=\omega n$. Note also that we have the natural correspondence $q=-i \partial_{x}$. We are interested in the roots of $P_{\mu}$, which we shall call $q_{n, j}, j=1, \ldots, 4$. To simplify the notation, we shall assume throughout $\mu>0$, the case of $\mu<0$ being equivalent.

Lemma 7.1. For sufficiently small $\eta>0$ and for $\mu>1.5$, the roots $q_{n, j}$ of $P_{\mu}$ satisfy

$$
\left|q_{n, j}\right|=\mathbf{O}(\mu / \eta) \text {. }
$$

For $\eta \mu \leqq \eta^{1 / 3}$, one has

$$
\left|\operatorname{Im} q_{n, j}\right| \geqq \mathbf{O}\left((\mu / \eta)^{1 / 2}\right),
$$

and

$$
\left|P_{\mu}^{\prime}\left(q_{n, j}\right)\right|=\mathbf{O}\left(\eta^{3 / 2} \mu^{1 / 2}\right) .
$$

For $\eta^{1 / 3}<\eta \mu<\eta^{-1 / 3}$, one has

$$
\left|\operatorname{Im} q_{n, j}\right| \geqq \mathbf{O}(1) \frac{\min \left((\eta \mu)^{1 / 4},(\eta \mu)^{1 / 2}\right)}{\eta},
$$

and

$$
\left|P_{\mu}^{\prime}\left(q_{n, j}\right)\right| \geqq \mathbf{O}\left(\eta^{3 / 2} \mu^{1 / 2}\right) .
$$

Finally, if $\eta \mu \geqq \eta^{-1 / 3}$, then

$$
\left|I m q_{n, j}\right| \geqq \mathbf{O}\left(\mu^{1 / 4} \eta^{-3 / 4}\right),
$$

and

$$
\left|P_{\mu}^{\prime}\left(q_{n, j}\right)\right|=\mathbf{O}\left(\eta(\eta \mu)^{3 / 4}\right) \geqq \mathbf{O}\left(\eta^{3 / 2} \mu^{1 / 2}\right) .
$$

Proof. We proceed by asymptotic analysis. Consider first the case $\eta \mu<\eta^{1 / 3}$. (The exponent $1 / 3$ is somewhat arbitrarily chosen. In fact, any number $<1 / 2$ would do for our purpose.) We set

$$
p=\mu+\eta q
$$


and study the polynomial

$$
R_{0}(p)=\eta^{2}-\left(1-p^{2}\right)^{2}+i c \eta p-i c \eta \mu .
$$

It is now useful to change variables by setting $p=\sigma+\eta / z$, where $\sigma \in\{+1,-1\}$. We shall pursue the case $\sigma=1$, the other one being analogous. Then we get the equation

$$
-z^{4}+(2 z+\eta)^{2}+i c z^{4} \frac{\mu-1}{\eta}-i c z^{3}=0 .
$$

Under the condition $\eta \mu<\eta^{1 / 3}$, the solutions of this equation are approximated by those of the equation

$$
i c z^{4}(\mu-1) / \eta+4 z^{2}=0 .
$$

Substituting back, we find that for small $\eta$ one has $p \approx \pm 1 \pm 2(i c \eta(\mu-1))^{1 / 2}$, i.e.

$$
q \approx \frac{ \pm 1-\mu}{\eta} \pm 2\left(i c \frac{(\mu-1)}{\eta}\right)^{1 / 2} .
$$

This implies (7.3) and (7.2) for the values of $\eta \mu$ considered thus far. We now note that $R_{0}^{\prime}(p)=4 p\left(1-p^{2}\right)+i c \eta$, from which (7.4) follows.

Next, we analyze the case $\eta \mu>\eta^{-1 / 3}$. Then it is adequate to change first variables as in (7.9), and to set then $p=1 / z$. The equation is now

$$
\eta^{2} z^{4}-\left(z^{2}-1\right)^{2}+i c \eta z^{3}-i c \eta \mu z^{4}=0 .
$$

Its solution is approximated by the solution of

$$
z^{4} i c \eta \mu=1
$$

Thus, we get

$$
q_{n, j} \approx \frac{-\mu+(i c \eta \mu)^{1 / 4}}{\eta},
$$

with a choice of four phases for the fourth root. Since $\eta \mu \gg 1$, and $\left|p_{n, j}\right|=\mathbf{O}\left((\eta \mu)^{1 / 4}\right)$, we see that

$$
\left|P_{\mu}^{\prime}\left(q_{n, j}\right)\right|=\eta\left|R_{0}^{\prime}\left(p_{n, j}\right)\right|=\mathbf{O}\left(\eta^{7 / 4} \mu^{3 / 4}\right) .
$$

The inequalities (7.2) and (7.7) follow.

Finally, consider $\eta^{1 / 3}<\eta \mu<\eta^{-1 / 3}$. With the change of variable (7.9), we are led to the approximate equation

$$
\left(p^{2}-1\right)^{2}+i c \eta \mu=0,
$$

whose solutions are

$$
\begin{aligned}
& p= \pm\left(1 \pm(i c \eta \mu)^{1 / 2}\right)^{1 / 2} . \\
& q=\frac{ \pm\left(1 \pm(i c \eta \mu)^{1 / 2}\right)^{1 / 2}-\mu}{\eta}
\end{aligned}
$$


The bound on $\operatorname{Im} q_{n, j}$ follows. We also have

$$
\left|P_{\mu}^{\prime}\left(q_{n, j}\right)\right| \approx 4\left|p_{n, j}\right|(c \eta \mu)^{1 / 2} \eta,
$$

by (7.18) and the explicit form of $R_{0}^{\prime}$. The required bounds follow now from obvious minorizations of the square root in (7.20) (and of its imaginary part). The lemma is proved.

Corollary 7.2. For sufficiently small $\eta>0$ and for $\mu>1.5$, one has the bounds

$$
\left|\operatorname{Im}\left(q_{n, j}\right) P_{\mu}^{\prime}\left(q_{n, j}\right)\right| \geqq \begin{cases}\mathbf{O}(\eta) & \text { if } \eta \mu \leqq \eta^{1 / 3} \\ \mathbf{O}\left(\eta \mu^{3 / 4}\right) & \text { if } \eta^{1 / 3}<\eta \mu<\eta^{-1 / 3} \\ \mathbf{O}\left((\eta \mu)^{3 / 4}\right) & \text { if } \eta \mu \geqq \eta^{-1 / 3}\end{cases}
$$

The following identities will be useful later.

Lemma 7.3. For $0 \leqq k \leqq 2$ one has

$$
\begin{gathered}
\sum_{j=1}^{4} \frac{q_{n, j}^{k}}{P_{\mu}^{\prime}\left(q_{n, j}\right)}=0, \\
\sum_{j=1}^{4} \frac{1}{\left(q-q_{n, j}\right) P_{\mu}^{\prime}\left(q_{n, j}\right)}=\frac{1}{P_{\mu}(q)} .
\end{gathered}
$$

Proof. We use contour integration. In the first case, we have

$$
\sum_{j=1}^{4} \frac{q_{n, j}^{k}}{P_{\mu}^{\prime}\left(q_{n, j}\right)}=\frac{1}{2 \pi i} \int_{|z|=r} \frac{d z z^{k}}{P_{\mu}(z)}=0,
$$

as is easily seen by letting $r$ tend to $\infty$. In the second case, we have, for sufficiently large $r$,

$$
\sum_{j=1}^{4} \frac{1}{\left(q-q_{n, j}\right) P_{\mu}^{\prime}\left(q_{n, j}\right)}=\frac{1}{2 \pi i} \int_{|z|=r} \frac{d z}{(q-z) P_{\mu}(z)}+\frac{1}{P_{\mu}(q)} .
$$

The assertion follows as before.

We now begin our study, for the case $n \neq \pm 1$, of the operator $A_{\omega n}^{-1}$ on the space $\mathbf{H}_{\alpha, X}$. Here, we fix, once and for all, a (large) positive constant $X$, which will be chosen adequately in later stages of the proof. The constant $\alpha$ is choosen larger than $\beta$, where $\beta$ is the rate of decay of $l^{\prime}$ near $-\infty$. Consider again the characteristic polynomial $P_{\mu}$ defined in (7.1). We number the roots of $P_{\mu}$ in such a way that $\operatorname{Im} q_{n, j}>0$ for $j=1,2$, $\operatorname{Im} q_{n, j}<0$ for $j=3,4$. For each $\mu$, and $X$ as above, we define an inverse $A_{\mu}^{-1}$ as follows.

$$
\left(A_{\mu}^{-1} f\right)(x)=\sum_{j=1,2} \int_{-\infty}^{x} d y \frac{e^{i q_{n, j}(x-y)}}{P_{\mu}^{\prime}\left(q_{n, j}\right)} f(y)-\sum_{j=3,4} \int_{x}^{x} d y \frac{e^{i q_{n, j}(x-y)}}{P_{\mu}^{\prime}\left(q_{n, j}\right)} f(y) .
$$

The norm on $\mathbf{H}_{\alpha, X}$ was defined by

$$
\|f\|_{\mathbf{H}_{\alpha, X}}=\sup _{x \in(-\infty, X]}|f(x)| m(x),
$$


where

$$
m(x)=e^{-\alpha x}+\frac{e^{x / 2}}{1+|x|} .
$$

Bounding $A_{\mu}^{-1}$ amounts thus to controlling expressions of the form

$$
m(x) \int_{-\infty}^{x} d y \frac{f(y) e^{i q_{n, j}(x-y)}}{P_{\mu}^{\prime}\left(q_{n, j}\right)}
$$

or

$$
m(x) \int_{x}^{X} d y \frac{f(y) e^{i q_{n, j}(y-x)}}{P_{\mu}^{\prime}\left(q_{n, j}\right)}
$$

We pursue the first case only. We are led to bound an integral of the form

$$
m(x) \int_{-\infty}^{x} d y\left|e^{i q_{n, j}(x-y)} / m(y)\right|,
$$

which by Lemma 6.2 is bounded by $\mathbf{O}\left(\left|\operatorname{Im} q_{n, j}\right|^{-1}\right)$. Thus the norm of $A_{\omega n}^{-1}$ is bounded by the inverse of $\left|\operatorname{Im}\left(q_{n, j}\right) P_{\mu}^{\prime}\left(q_{n, j}\right)\right|$ which, by Corollary 7.2, is bounded below by $\mathbf{O}(\eta)$. Thus we see that

$$
\left\|A_{\mu}^{-1} f\right\|_{\mathbf{H}_{\alpha, X}} \leqq \mathbf{O}\left(\eta^{-1}\right)\|f\|_{\mathbf{H}_{\alpha, X}} .
$$

Note that somewhat better bounds are possible by using the explicit form of Corollary 7.2.

We shall need the following better bound which holds when $f$ is differentiable.

Lemma 7.4. If $f$ is once continuously differentiable then one has

$$
\left\|A_{\mu}^{-1} f\right\|_{\mathbf{H}_{\alpha, X}} \leqq \mathbf{O}(1)\left(\eta^{-1 / 2}\|f\|_{\mathbf{H}_{\alpha, X}}+\left\|f^{\prime}\right\|_{\mathbf{H}_{\alpha, X}}\right) .
$$

Proof. We rewrite (7.25) as follows

$$
\left(A_{\mu}^{-1} f\right)(x)=\sum_{j=1,2} \int_{-\infty}^{x} d y \frac{\partial_{y} e^{i q_{n, j}(x-y)}}{-i q_{n, j} P_{\mu}^{\prime}\left(q_{n, j}\right)} f(y)-\sum_{j=3,4} \int_{x}^{x} d y \frac{\partial_{y} e^{i q_{n, j}(x-y)}}{-i q_{n, j} P_{\mu}^{\prime}\left(q_{n, j}\right)} f(y),
$$

and then integrate by parts. We obtain

$$
\begin{aligned}
\left(A_{\mu}^{-1} f\right)(x)= & -\sum_{j=1,2} \int_{-\infty}^{x} d y \frac{e^{i q_{n, j}(x-y)}}{-i q_{n, j} P_{\mu}^{\prime}\left(q_{n, j}\right)} f^{\prime}(y) \\
& +\sum_{j=3,4} \int_{x}^{x} d y \frac{e^{i q_{n, j}(x-y)}}{-i q_{n, j} P_{\mu}^{\prime}\left(q_{n, j}\right)} f^{\prime}(y) \\
& +\sum_{j=1}^{4} \frac{f(x)}{-i q_{n, j} P_{\mu}^{\prime}\left(q_{n, j}\right)}-\sum_{j=3,4} \frac{f(X) e^{i q_{n, j}(x-X)}}{-i q_{n, j} P_{\mu}^{\prime}\left(q_{n, j}\right)} .
\end{aligned}
$$

The boundary terms at $x$ can be bounded using (7.24) (for $q=0$ ) and contribute 
$\mathbf{O}(1)\|f\|_{\mathbf{H}_{\alpha, X}}$. The norm of the boundary terms at $X$ is bounded by

$$
\sum_{j=3,4} \frac{1}{\left|q_{n, j} P_{\mu}^{\prime}\left(q_{n, j}\right)\right|} \frac{m(x)}{m(X)} e^{-\left|\operatorname{Im} q_{n, j}\right|(X-x)}\|f\|_{\mathbf{H}_{\alpha, X}} .
$$

Using Lemma 6.3, and Lemma 7.1, this leads to a bound

$$
\mathbf{O}\left(\eta^{-1 / 2}\right)\|f\|_{\mathbf{H}_{\alpha, X}} \text {. }
$$

Combining these bounds with a bound on the integrals in (7.29) which is the same as the one leading from (7.25) to (7.27), we get

$$
\left\|A_{\mu}^{-1} f\right\|_{\mathbf{H}_{\alpha, X}} \leqq \mathbf{O}(1)\left(\eta^{-1 / 2}\|f\|_{\mathbf{H}_{\alpha, X}}+\left\|f^{\prime}\right\|_{\mathbf{H}_{\alpha, X}}\right) .
$$

We next discuss the roots of the polynomial $P_{\omega}$ (defined in (7.1)) for the case $n=$ \pm 1 . Two of the roots may be confluent in this case. We write

$$
\omega=1-\rho \eta^{2}, \quad c=4+\eta \tau .
$$

Setting $q=i / 2+y$, we get the equation

$$
4 y^{2}+\eta(-i / 2+\tau / 2-4 i \rho+\mathbf{O}(y))+\eta^{2}(\mathbf{O}(y)+\mathbf{O}(1))=0 .
$$

This implies that there are two roots, possibly equal, satisfying $|y| \leqq \mathbf{O}\left(\eta^{1 / 2}\right)$, so that the first two roots of $P_{\omega}$ are

$$
q_{1, j}=\frac{i}{2}+\mathbf{O}\left(\eta^{1 / 2}\right), \quad \text { for } j=1,2
$$

To locate the two other roots, we set $\eta q+\omega=-\omega-y$. The equation for $y$ is then

$$
\eta^{2}-\left(1-(\omega+y)^{2}\right)^{2}-2 i c \eta \omega-i c \eta y=0 .
$$

The small roots of this equation are approximated by those of

$$
-4 y^{2}-i c \eta y-2 i c \eta=0
$$

since $\omega-1=\mathbf{O}\left(\eta^{2}\right)$. Thus we find

$$
q_{1, j}=-\frac{2}{\eta} \pm\left(\frac{-i c}{2 \eta}\right)^{1 / 2}+\mathbf{O}(1), \quad \text { for } j=3,4 \ldots
$$

The following result will be useful in Sect. 13.

Lemma 7.5. For $0 \leqq k \leqq 3$, and $f \in \mathbf{H}_{\alpha, X}$, the function $\partial_{x}^{k} A_{\mu}^{-1} f$ exists and has the representation

$$
\begin{aligned}
\left(\partial_{x}^{k} A_{\mu}^{-1} f\right)(x)= & \sum_{j=1,2} \int_{-\infty}^{x} d y\left(i q_{n, j}\right)^{k} \frac{e^{i q_{n, j}(x-y)}}{P_{\mu}^{\prime}\left(q_{n, j}\right)} f(y) \\
& -\sum_{j=3,4} \int_{x}^{X} d y\left(i q_{n, j}\right)^{k} \frac{e^{i q_{n, j}(x-y)}}{P_{\mu}^{\prime}\left(q_{n, j}\right)} f(y) .
\end{aligned}
$$

Proof. Consider the definition (7.25) of $A_{\mu}^{-1}$. The assertion follows now readily by differentiating this expression and observing that the boundary terms at $x$ drop because of Lemma 7.3. 
We next study the operator $A_{\omega n}$ acting on quadruples of derivatives. Assume $f(x)=e^{i q x}$. Then

$$
\left(A_{\omega n} f\right)(x)=P_{\omega n}(q) e^{i q x},
$$

where the characteristic polynomial of the differential operator $A_{\omega n}$ is given, as in (7.1), by

$$
P_{\omega n}(q)=\eta^{2}-\left(1-(\omega n+\eta q)^{2}\right)^{2}+i \eta^{2} c q .
$$

Denote $q_{n, j} j=1, \ldots, 4$, the roots of $P_{\omega n}$. To each function $f \in C^{3}$, we associate the quadruple:

$$
f(x), i^{-1} f^{\prime}(x), i^{-2} f^{\prime \prime}(x), i^{-3} f^{\prime \prime \prime}(x) .
$$

If we write $\langle\cdots|$ for row vectors and $|\cdots\rangle$ for column vectors, we see that the eigenvectors $e^{i q_{n, j} x}$ of $A_{\omega n}$ are mapped to $\left|1, q_{n, j}, q_{n, j}^{2}, q_{n, j}^{3}\right\rangle$. The projection $P_{n}^{(j)}$ onto the eigenvector

$$
\left|e_{n, j}\right\rangle=\left|1, q_{n, j}, q_{n, j}^{2}, q_{n, j}^{3}\right\rangle
$$

is given by

$$
P_{n}^{(j)}=\left|e_{n, j}\right\rangle\left\langle P_{n, 0}^{(j)}, P_{n, 1}^{(j)} P_{n, 2}^{(j)}, P_{n, 3}^{(j)}\right|
$$

where

$$
\sum_{m=0}^{3} P_{n, m}^{(j)} q^{m}=\frac{P_{\omega n}(q)}{\left(q-q_{n, j}\right) P_{\omega n}^{\prime}\left(q_{n, j}\right)}
$$

With these definitions, it is easy to check that

$$
P_{n}^{(j)} e_{n, k}=\delta_{j, k} e_{n, j}, \quad \text { for all } j, k=1, \ldots, 4 \text {. }
$$

We now formulate bounds on the $P_{n, m}^{(j)}$.

Lemma 7.6. For $n \neq 1, m=0, \ldots, 3$, and $j=1, \ldots, 4$, one has

$$
\left|P_{n, m}^{(j)}\right| \leqq \mathbf{O}(1) n^{5 / 2-m} \eta^{-1 / 2+m}
$$

Proof. First we note that by (7.43), and (7.2) one has

$$
P_{\omega n}(q)=-\eta^{4} \prod_{j=1}^{4}\left(q-q_{n, j}\right)
$$

and hence

$$
\left|P_{\omega n}^{\prime}\left(q_{n, j}\right) P_{n, m}^{(j)}\right| \leqq \eta^{4} \mathbf{O}\left(\sup _{k=1, \ldots, 4}\left|q_{n, k}\right|\right)^{3-m} \leqq \eta^{4} \mathbf{O}\left(\frac{\mu}{\eta}\right)^{3-m} .
$$

Similarly, using (7.4), (7.6), and (7.8), we see that

$$
1 /\left|P_{\omega n}^{\prime}\left(q_{n, j}\right)\right| \leqq \mathbf{O}\left(\eta^{-3 / 2} \mu^{-1 / 2}\right) \text {. }
$$

Thus,

$$
\left|P_{n, m}^{(j)}\right| \leqq \mathbf{O}(1) n^{5 / 2-m} \eta^{-1 / 2+m}
$$


as asserted.

Lemma 7.7. When $n=1$ one has

$$
\left|P_{1, m}^{(1)}+P_{1, m}^{(2)}\right| \leqq \begin{cases}\mathbf{O}(1) & \text { if } m=0 \\ \mathbf{O}\left(\eta^{3 / 2}\right) & \text { if } m=1,2 \\ \mathbf{O}\left(\eta^{5 / 2}\right) & \text { if } m=3\end{cases}
$$

Moreover,

$$
\left|P_{1, m}^{(3)}\right|+\left|P_{1, m}^{(4)}\right| \leqq \mathbf{O}(1)
$$

and

$$
\left|\left(q_{1,2}-q_{1,1}\right) P_{1, m}^{(2)}\right| \leqq \mathbf{O}(1) .
$$

Proof. Note that when $n=1$, the bounds on $P_{1}^{(1)}$ and $P_{1}^{(2)}$ diverge because two roots (say $q_{1,1}$ and $q_{1,2}$ ) can be arbitrarily close and could even coincide. However, $P_{1}^{(1)}+P_{1}^{(2)}$ stays bounded, as we show now. Let us denote $q_{1,1}, q_{1,2}$ the two roots near $i / 2$, and $q_{1,3}, q_{1,4}$ the two other roots. By the definition of $P_{1, m}^{(j)}$, we have

$$
\sum_{m=0}^{3}\left(P_{1, m}^{(1)}+P_{1, m}^{(2)}\right) q^{m}=P_{\omega}(q)\left\{\frac{1}{\left(q-q_{1,1}\right) P_{\omega}^{\prime}\left(q_{1,1}\right)}+\frac{1}{\left(q-q_{1,2}\right) P_{\omega}^{\prime}\left(q_{1,2}\right)}\right\} .
$$

By the identity (7.24), we see that this is equal to

$$
-P_{\omega}(q)\left\{\frac{1}{\left(q-q_{1,3}\right) P_{\omega}^{\prime}\left(q_{1,3}\right)}+\frac{1}{\left(q-q_{1,4}\right) P_{\omega}^{\prime}\left(q_{1,4}\right)}\right\}+1 .
$$

Note now that for $j=3,4$, one has $\left|P_{\omega}^{\prime}\left(q_{1, j}\right)\right|=\mathbf{O}\left(\eta^{4} \eta^{-2} \eta^{-1 / 2}\right)$, by (7.35) and (7.38). Therefore, we find from (7.53) and (7.54) the bound (7.50). The proof of Eq. (7.51) is easier and is left to the reader. To prove (7.52), we note

$$
\frac{P_{\omega}(q)}{\left(q-q_{1,2}\right) P_{\omega}^{\prime}\left(q_{1,2}\right)}=\frac{1}{q_{1,2}-q_{1,1}} \cdot \frac{\left(q-q_{1,1}\right)\left(q-q_{1,3}\right)\left(q-q_{1,4}\right)}{\left(q_{1,2}-q_{1,3}\right)\left(q_{1,2}-q_{1,4}\right)},
$$

and hence, by (7.35) and (7.38),

$$
\left|\left(q_{1,2}-q_{1,1}\right) P_{m}^{(2)}\right| \leqq \mathbf{O}\left(\eta^{-2} / \eta^{-2}\right)=\mathbf{O}(1) .
$$

The lemma is proven. For further use, we also note two identities which are easily derived from (7.39)

$$
\begin{aligned}
\sum_{k=0}^{3}\left(P_{n, k}^{(l)} \partial_{x}^{k} A_{\omega n}^{-1} f\right)(x)= & \sum_{k=0}^{3}\left(\sum_{j=1,2} \int_{-\infty}^{x} d y \frac{P_{n, k}^{(l)} q_{n, j}^{k} e^{i q_{n, j}(x-y)}}{P_{\mu}^{\prime}\left(q_{n, j}\right)} f(y)\right. \\
& \left.-\sum_{j=3,4} \int_{x}^{X} d y \frac{P_{n, k}^{(l)} q_{n, j}^{k} e^{i q_{n, j}(x-y)}}{P_{\mu}^{\prime}\left(q_{n, j}\right)} f(y)\right) .
\end{aligned}
$$

By (7.43), this projects onto the term with $j=l$. More precisely, this means e.g. in the case $l=1$, that

$$
\sum_{k=0}^{3}\left(P_{n, k}^{(1)} \partial_{x}^{k} A_{\omega n}^{-1} f\right)(x)=\int_{-\infty}^{x} d y \frac{e^{i q_{n, 1}(x-y)}}{P_{\mu}^{\prime}\left(q_{n, 1}\right)} f(y)
$$




\section{A Stable Manifold Theorem for Maps with Unbounded Linear Part}

This section deals with the existence of a stable manifold in the case when the linear part of the evolution equation is unbounded. We shall use this theory to control the front problem at $x=+\infty$. Here, we consider, first on a purely formal level, an equation of the form

$$
\frac{d X}{d t}=A X+F(X)
$$

with $X$ in some separable Banach space $\mathbf{B}, A$ unbounded, but with spectrum away from the imaginary axis, $F$ maps a neighborhood of 0 in $\mathbf{B}$ to $\mathbf{B}$ and is a twice Fréchet differentiable function satisfying $F(0)=0, F^{\prime}(0)=0$.

We want to study Eq. (8.1); however if $A$ is truly unbounded above, then the flow itself is not defined for positive $t$. We shall nevertheless be able to define a stable manifold $W^{s}$, tangent to the "linearly stable space" $E^{s}$, i.e. the spectral sub-space of $A$ corresponding to the left half-plane of its spectrum. We denote $E^{u}$ the unstable subspace and we assume that $P^{s}$, and $P^{u}$, the respective spectral projections, are bounded operators.

The construction of $W^{s}$ is possible because it can be formulated in terms of the semigroups $e^{A^{s_{\tau}}}, e^{-A^{u_{\tau}}}, \tau \geqq 0$, and these semigroups are defined. Here, $A^{s}=P^{s} A$, and $A^{u}=P^{u} A$. In order to formulate the problem in terms of these semigroups, we consider evolution equations on the stable manifold itself.

As usual, $W^{s}$ will be viewed as the graph of a function $\Phi: E^{s} \rightarrow E^{u}$, and in fact we shall prove the existence of $\Phi$ on a small ball $B_{r}^{s}$ in $E^{s}$, (of radius $r$, centered at 0 ). We need to control the flow on $W^{s}$ (where it will be seen to be defined for all $t \geqq 0$ ), and we denote $\psi_{t}^{\Phi}$ the solution (for fixed $\Phi$ ) of

$$
\frac{d}{d t} \psi_{t}^{\Phi}=A^{s} \psi_{t}^{\Phi}+G_{\Phi^{\circ}}^{s} \psi_{t}^{\Phi}
$$

where $\psi_{t}^{\Phi}: E^{s} \rightarrow E^{s}$, and

$$
G_{\Phi}^{s}(y)=P^{s} F(y, \Phi(y)), \quad y \in E^{s} .
$$

This is the flow in $W^{s}$, projected onto $E^{s}$. The solution of (8.2) is formally given by

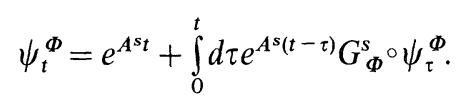

The flow $\phi_{t}$ on $W^{s}$ itself must be of the form

$$
\phi_{t}\left(\begin{array}{c}
x \\
\Phi(x)
\end{array}\right)=\left(\begin{array}{c}
\psi_{t}^{\Phi}(x) \\
\Phi\left(\psi_{t}^{\Phi}(x)\right)
\end{array}\right)
$$

which, upon differentiation, and restriction to the second component, leads to the equation (on $E^{s}$ )

$$
\left.D \Phi_{x} \cdot \frac{d}{d t} \psi_{t}^{\Phi}\right|_{t=0}=A^{u} \Phi+G_{\Phi}^{u}
$$


where $G_{\Phi}^{u}(x)=P^{u} F\left(\begin{array}{c}x \\ \Phi(x)\end{array}\right)$.

Equation (8.4) is equivalent to

$$
\Phi=-\int_{0}^{\infty} d \tau e^{-A^{u} \tau} G_{\Phi}^{u} \psi_{\tau}^{\Phi}
$$

We shall solve the existence problem for the stable manifold by considering the system (8.3), (8.5), using techniques similar to those found in [5].

Given $\Phi$, we solve (8.3), and this will define the right-hand side of (8.5), i.e. a map $\Phi \rightarrow C(\Phi)$. We shall show that the operator $C$ is a contraction of a sufficiently small ball of functions, so that (8.5) has a solution by the contraction mapping principle.

We now describe in detail the assumptions on $A$. In fact, $A$ derives from two semigroups, corresponding intuitively to the positive and the negative parts of the spectrum of $A$. The Banach space $\mathbf{B}$ is a direct sum $\mathbf{B}=E^{s} \oplus E^{u}$. By $P^{s}$ and $P^{u}$, we denote the corresponding projections. We assume that $A^{s}$ and $-A^{u}$ are the generators of two equicontinuous semigroups of Class $C_{0}$ in the sense of Yosida [6], on $E^{s}$ and $E^{u}$ respectively. Finally, $A$ is the direct sum of $A^{s}$ and $A^{u}$ on their respective domains in $E^{s}$ and $E^{u}$. Furthermore, we assume there is a constant $D>0$ such that the semigroups satisfy the bounds

$$
\sup _{t \geqq 0} \max \left(e^{\lambda^{s t}}\left\|e^{A^{s_{t}}}\right\|_{E^{s}}, e^{\lambda^{u_{t}}}\left\|e^{-A^{u_{t}}}\right\|_{E^{u}}\right) \leqq D
$$

with $\lambda^{s}>0, \lambda^{u}>0$.

We make the following continuity and differentiability assumptions on $F$ : With the same constant $D$ as above (for convenience) one has

$$
\begin{aligned}
\|F(x, y)\| & \leqq D \max \left(\|x\|_{s},\|y\|_{u}\right)^{2}, \\
\left\|D F(x, y)\left(x^{\prime}, y^{\prime}\right)\right\| & \leqq D \max \left(\|x\|_{s},\|y\|_{u}\right) \max \left(\left\|x^{\prime}\right\|_{s}, y^{\prime} \|_{u}\right),
\end{aligned}
$$

on a neighborhood of $(0,0)$.

We shall fix below a small $r>0$, and a $\sigma>0$. We write $B_{r}^{u}$ for the ball of radius $r$ in $E^{u}$ centered at 0 , similarly $B_{r}^{s}$ for the ball in $E^{s}$. We define

$$
\begin{aligned}
\mathbf{A}_{r, \sigma}= & \left\{\Phi: B_{r}^{s} \rightarrow E^{u}, \Phi(0)=0,\right. \\
& \left.\|\Phi(x)-\Phi(y)\|_{u} \leqq \sigma\|x-y\|_{s}, \quad \text { for } x, y \in B_{r}^{s}\right\} .
\end{aligned}
$$

We also define, for $\Phi, \Psi \in \mathbf{A}_{r, \sigma}$, the Lipshitz distance

$$
d_{\mathbf{A}_{r}}(\Phi, \Psi)=\sup _{\substack{x \in B_{r}^{s} \\ x \neq 0}} \frac{\|\Phi(x)-\Psi(x)\|_{u}}{\|x\|_{s}} .
$$

Theorem 8.1. Under the above assumptions, the operator $C$ has for every $\sigma>D$ and for every sufficiently small $r>0$ a unique fixed point in $\mathbf{A}_{r, \sigma}$.

Proof. For every $\Phi \in \mathbf{A}_{r, \sigma}$, we first show the existence and study the properties of $\psi^{\Phi}$, 
solving (8.3). We want to describe $\psi^{\Phi}$ as an element of a set $\mathbf{B}_{r, \sigma}$ of functions

$$
\begin{aligned}
\mathbf{B}_{r, \sigma}= & \left\{\psi: \mathbb{R}^{+} \times B_{r}^{s} \rightarrow B_{r}^{s} \mid \psi_{0}(x)=x, \quad \psi_{t}(0)=0 \quad \text { for all } t>0,\right. \\
& \left.\sup _{t \geqq 0}\left\|\psi_{t}(x)-\psi_{t}(y)\right\|_{s} \leqq \sigma\|x-y\|_{s}\right\} .
\end{aligned}
$$

We equip the set $\mathbf{B}_{r, \sigma}$ with a Lipshitz distance

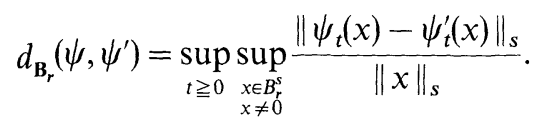

It is easy to verify that $\mathbf{B}_{r . \sigma}$ is a complete metric space for this distance.

We first study Eq. (8.3). Assume that $\psi \in \mathbf{B}_{r, \sigma}$, and $\Phi \in \mathbf{A}_{r, \sigma}$ and denote $H_{\Phi}(\psi)$ the right-hand side of (8.3), i.e.,

$$
H_{\Phi}(\psi)_{t}=e^{A^{s} t}+\int_{0}^{t} d \tau e^{A^{s}(t-\tau)} G_{\Phi}^{s} \psi_{\tau} .
$$

We shall show that $H_{\Phi}$ is a contraction in $\mathbf{B}_{r, \sigma}$ if $r$ is sufficiently small. From

$$
\begin{gathered}
\left\|\psi_{\tau}(x)-\psi_{\tau}(y)\right\|_{s} \leqq \sigma\|x-y\|_{s} \\
\left\|\Phi\left(\psi_{\tau}(x)\right)-\Phi\left(\psi_{\tau}(y)\right)\right\|_{u} \leqq \sigma\left\|\psi_{\tau}(x)-\psi_{\tau}(y)\right\|_{s},
\end{gathered}
$$

and

$$
\left\|\Phi\left(\psi_{\tau}(x)\right)\right\|_{u} \leqq \sigma\left\|\psi_{\tau}(x)\right\|_{s} \leqq \sigma^{2}\|x\|_{s}
$$

we deduce

$$
\max \left(\left\|\psi_{\tau}(x)\right\|_{s},\left\|\Phi\left(\psi_{\tau}(x)\right)\right\|_{u}\right) \leqq \max \left(\left\|\psi_{\tau}(x)\right\|_{s}, \sigma\left\|\psi_{\tau}(x)\right\|_{s}\right) \leqq\left(\sigma+\sigma^{2}\right) r .
$$

Therefore, we have

$$
\left\|H_{\Phi}(\psi)_{t}(x)-H_{\Phi}(\psi)_{t}(y)\right\|_{s} \leqq\left(D e^{-\lambda^{s} t}+\int_{0}^{t} d \tau e^{-\lambda^{s}(t-\tau)} D^{2} r\left(\sigma+\sigma^{2}\right)^{2}\right)\|x-y\|_{s} .
$$

This implies $H_{\Phi}(\psi) \in \mathbf{B}_{r, \sigma}$ if

$$
D+\frac{r D^{2}\left(\sigma+\sigma^{2}\right)^{2}}{\lambda_{s}}<\sigma
$$

which is always possible if $\sigma>D$, and $r>0$ is sufficiently small.

We now show that $H_{\Phi}$ is a contraction. We have

$$
\begin{aligned}
& \left\|H_{\Phi}(\psi)_{t}(x)-H_{\Phi}\left(\psi^{\prime}\right)_{t}(x)\right\|_{s} \\
& \quad \leqq \int_{0}^{t} d \tau e^{-\lambda^{s(t-\tau)} D^{2}(1+\sigma)\left(\sigma+\sigma^{2}\right) r\left\|\psi_{\tau}(x)-\psi_{\tau}^{\prime}(x)\right\|_{s}} \\
& \quad \leqq \sigma r D^{2}(1+\sigma)^{2}\|x\|_{s} \int_{0}^{t} d \tau e^{-\lambda^{s}(t-\tau)} d_{\mathbf{B}_{r}}\left(\psi, \psi^{\prime}\right) \\
& \quad \leqq \sigma r D^{2}(1+\sigma)^{2}\|x\|_{s} \frac{1}{\lambda^{s}} d_{\mathbf{B}_{r}}\left(\psi, \psi^{\prime}\right) \leqq \frac{1}{2}\|x\|_{s} d_{\mathbf{B}_{r}}\left(\psi, \psi^{\prime}\right),
\end{aligned}
$$


provided

$$
2 \sigma r D^{2}(1+\sigma)^{2}<\lambda^{s}
$$

Therefore, $H_{\Phi}$ is a contraction and has a unique fixed point in $\mathbf{B}_{r, \sigma}$. This fixed point is called $\psi^{\Phi}$.

We next study the map $C$ defined by the right-hand side of (8.5). We first show that $C$ maps $\mathbf{A}_{r, \sigma}$ into itself. If $\Phi \in \mathbf{A}_{r, \sigma}$, then we have, as before,

$$
\left\|\Phi \circ \psi_{\tau}^{\Phi}(x)-\Phi \circ \psi_{\tau}^{\Phi}(y)\right\|_{u} \leqq \sigma^{2}\|x-y\|_{s},
$$

and therefore,

$$
\begin{aligned}
\|C(\Phi)(x)-C(\Phi)(y)\|_{u} & \leqslant \frac{D^{2}\left(\sigma+\sigma^{2}\right)^{2} r}{\lambda^{u}}\|x-y\|_{s}, \\
& \leqq \sigma\|x-y\|_{s},
\end{aligned}
$$

for sufficiently small $r$. In other words, $C$ maps $\mathbf{A}_{r, \sigma}$ to itself. We next show that $C$ is a contraction. Note that

$$
G_{\Phi^{\circ}}^{u} \psi_{\tau}^{\Phi}-G_{\Psi^{\circ}}^{u} \psi_{\tau}^{\Psi^{\Psi}}=\left(G_{\Phi^{\circ}}^{u} \psi_{\tau}^{\Phi}-G_{\Phi^{\circ}}^{u} \psi_{\tau}^{{ }^{\Psi}}\right)+\left(G_{\Phi^{\circ}}^{u} \psi_{\tau}^{{ }_{\tau}}-G_{\Psi^{\circ}}^{u} \psi_{\tau}{ }^{\Psi}\right) .
$$

Using estimates of the same form as above, we find

$$
\begin{aligned}
\|C(\Phi)(x)-C(\Psi)(x)\|_{u} \leqq & \frac{D^{2}\left(\sigma+\sigma^{2}\right) r}{\lambda^{u}}\left((1+\sigma) \sup _{\tau \geqq 0}\left\|\psi_{\tau}^{\Phi}(x)-\psi_{\tau}^{\Psi}(x)\right\|_{s}\right. \\
& \left.+\sup _{\tau \geqq 0}\left\|\Phi\left(\psi_{\tau}^{\Psi}(x)\right)-\Psi\left(\psi_{\tau}^{\Psi}(x)\right)\right\|_{u}\right) . \\
\leqq & \frac{D^{2}\left(\sigma+\sigma^{2}\right) r}{\lambda^{u}}\left((1+\sigma) \sup _{\tau \geqq 0}\left\|\psi_{\tau}^{\Phi}(x)-\psi_{\tau}^{\Psi}(x)\right\|_{s}\right. \\
& \left.+\|x\|_{s} d_{A_{r}}(\Phi, \Psi)\right) .
\end{aligned}
$$

In order to estimate $\psi_{\tau}^{\Phi}(x)-\psi_{\tau}^{\Psi}(x)$ we use Eq. (8.3). We have

$$
\left\|\psi_{t}^{\Phi}(x)-\psi_{t}^{\Psi}(x)\right\|_{s} \leqq \int_{0}^{t} d \tau e^{-\lambda^{s}(t-\tau)}\left\|G_{\Phi^{\circ}}^{s} \psi_{\tau}^{\Phi}(x)-G_{\Psi^{\circ}}^{s} \psi_{\tau}^{\Psi}(x)\right\|_{s} .
$$

From this we get

$$
\begin{aligned}
\sup _{t}\left\|\psi_{t}^{\Phi}(x)-\psi_{t}^{\Psi}(x)\right\|_{s} \leqq & \frac{D^{2}\left(\sigma+\sigma^{2}\right) r}{\lambda^{s}} \\
& \cdot\left((1+\sigma) \sup _{\tau \geqq 0}\left\|\psi_{\tau}^{\Phi}(x)-\psi_{\tau}^{\Psi}(x)\right\|_{s}+\|x\|_{s} d_{A_{r}}(\Phi, \Psi)\right) .
\end{aligned}
$$

If $D^{2}\left(\sigma+\sigma^{2}\right)(1+\sigma) r<\lambda^{s} / 2$, then we get

$$
\sup _{t}\left\|\psi_{\tau}^{\Phi}(x)-\psi_{\tau}^{\Psi}(x)\right\|_{s} \leqq 2 \frac{D^{2}\left(\sigma+\sigma^{2}\right) r}{\lambda^{s}}\|x\|_{s} d_{A_{r}}(\Phi, \Psi),
$$

and taking $r$ smaller, if necessary, we see that $C$ is a contraction. Therefore, $C$ has a fixed point, as asserted.

A straightforward extension of the above methods, cf. [5], shows that if $F$ is $k$ 
times Fréchet differentiable, then so is $\Phi$. Note that this bound will always contain a factor $r$.

We now apply the above theory to Eq. (4.10) near $+\infty$, calling the space variable $t$ instead of $x$. We define the space B through the following construction. To each function $f \in C^{3}$, we associate the quadruple:

$$
f(t), i^{-1} f^{\prime}(t), i^{-2} f^{\prime \prime}(t), i^{-3} f^{\prime \prime \prime}(t) \text {. }
$$

Given a quadruple $f_{0}, \ldots, f_{3}$ (e.g. the quadruple associated to a function $f \in \mathscr{C}^{3}$ as above), we define a decomposition

$$
\left(f_{0}, \ldots, f_{3}\right)=b_{n}^{s}+b_{n}^{u},
$$

where

$$
b_{n}^{s}=\sum_{j=1}^{2} b_{n, j} e_{n, j}, \quad b_{n}^{u}=\sum_{j=3}^{4} b_{n, j} e_{n, j},
$$

and with $e_{n, j}$ as defined in Sect. 7. When $n=1$, the first sum extends from 1 to 3 and the second over $j=4$ only. We can decompose naturally $b_{n}^{s}$ (or $b_{n}^{u}$ ) as a quadruple, namely

$$
b_{n, m}^{s}=\sum_{j=1}^{2} b_{n, j} q_{n, j}^{m}, \quad m=0,1,2,3
$$

We define

$$
\left|b_{n}^{s}\right|_{n, *}=\sup _{0 \leqq m \leqq 3} b_{n, m}^{s} \gamma_{n}^{-m},
$$

where $\gamma_{n}=|\omega n| / \eta$ (an approximation to the root of maximum modulus of (7.1)). We define analogously $\left|b_{n}^{u}\right|_{n, *}$, and when $n=1$, the first sum extends from 1 to 3 and the second over $j=4$ only.

We now define $\mathbf{B}$ as the space of sequences of pairs

$$
b_{n}=\left(b_{n}^{s}, b_{n}^{u}\right)
$$

as above. We equip $\mathbf{B}$ with the norm

$$
\|\mathbf{b}\|_{\mathbf{B}}=\sup _{n} \eta^{-\rho_{1} n} n^{3} \max \left(\left|b_{n}^{s}\right|_{n, *},\left|b_{n}^{u}\right|_{n, *}\right),
$$

where $\rho_{1}>0$ will be fixed later.

The operator $A$ of Eq. (8.1) is formally defined by

$$
A e_{n, j}=q_{n, j} e_{n, j},
$$

i.e. $A$, restricted to the component $n$ of $\mathbf{B}$ is really $\eta^{-4} A_{\omega n}$. Associated with this definition there is a natural decomposition of $\mathbf{B}$ into $E^{s}$ and $E^{u}$. If we number the $q_{n, j}$ in such a way that $\operatorname{Im} q_{n, j}>0$ for $j=1,2$ when $n \neq \pm 1($ and for $j=1,2,3$ when $n=1)$, then

$$
E^{s}=\left\{\mathbf{b} \mid b_{n, j}=0 \text { for } j \neq 1,2(\text { and } 3 \text { when } n=1)\right\}
$$


It follows at once from the bounds on the $q_{n, j}$ in Sect. 7 that (8.6) holds with $\lambda^{s}$, $\lambda^{u}>1 / 4$, and $D=1$.

We next analyze the nonlinear term $F$ in Eq. (8.1). To an element $\mathbf{b} \in \mathbf{B}$ and a value of $t$ there corresponds a value of $W_{n}$ at $t$. In fact, we have

$$
W_{n}(t)=\sum_{j=1}^{4} b_{n, j}
$$

Note now that by virtue of Eq. (4.10), we have

where

$$
F_{n}=\sum_{j=1}^{4} F_{n, j} e_{n, j}=\left|0,0,0, N_{n}\right\rangle
$$

$$
N_{n}=\eta^{-2} \sum_{p+q+r=n} W_{p} W_{q} W_{r}
$$

We now bound this expression. Note that the sum over $p+q+r=n$ above can be absorbed through the norm on $\mathbf{B}$ :

$$
\sum_{\substack{p+q+r=n \\ p, q, r \neq 0}} \frac{\eta^{\rho_{1}(|p|+|q|+|r|-|n|)}}{p^{3} q^{3} r^{3}} \leqq \mathbf{O}\left(n^{-3}\right),
$$

uniformly in $\eta$. This leads to

$$
\left|N_{n}\right| \leqq \mathbf{O}(1) \eta^{-2+\rho_{1} n} n^{-3}\|\mathbf{b}\|_{\mathbf{B}}^{3} .
$$

We now note that

$$
F_{n, j} e_{n, j}=P_{n}^{(j)}\left|0,0,0, N_{n}\right\rangle=e_{n, j} P_{n, 3}^{(j)} N_{n} .
$$

We use now the bounds on $P_{n, m}$ given in Lemma 7.1 and 7.6. We start with the case $n \neq \pm 1$. We have

$$
\left|F_{n, m}^{s}\right| \leqq\left(\left|P_{n, 3}^{(1)} q_{n, 1}^{3}\right|+\left|P_{n, 3}^{(2)} q_{n, 2}^{3}\right|\right) \delta_{m, 3}\left|N_{n}\right|
$$

so that

$$
\begin{aligned}
\left|F_{n}^{s}\right|_{n, *} & \leqq \mathbf{O}(1) n^{-1 / 2} \eta^{5 / 2} n^{3} \eta^{-3} n^{-3} \eta^{-2+\rho_{1} n}\|\mathbf{b}\|_{\mathbf{B}}^{3} \\
& =\mathbf{O}(1) n^{-1 / 2} \eta^{-5 / 2+\rho_{1} n^{-3}} n^{-3}\|\mathbf{b}\|_{\mathbf{B}}^{3} .
\end{aligned}
$$

We next analyze the case $n=1$. We consider, as in Sect. 7, the operator

$$
P_{1}^{(1,2)}=\sum_{j=1}^{2}\left|e_{j}\right\rangle\left\langle P_{1,0}^{(j)}, P_{1,1}^{(j)}, P_{1,2}^{(j)}, P_{1,3}^{(j)}\right|
$$

where $P_{1, m}^{(j)}$ is defined by (7.43). We estimate similarly $P_{1}^{(3)}$ and $P_{1}^{(4)}$, but we give details only for $P_{1}^{(1,2)}$. Writing $P_{1}^{(1,2)}$ as $\left|e_{1,1}\right\rangle\left\langle e_{1,1}^{*}|+| e_{1,2}\right\rangle\left\langle e_{1,2}^{*}\right|$, we may estimate this quantity conveniently by considering

$$
\left|e_{1,1}\right\rangle\left\langle e_{1,1}^{*}+e_{1,2}^{*}|+| e_{1,2}-e_{1,1}\right\rangle\left\langle e_{1,2}^{*}\right| .
$$

We first look at $e_{1,1}^{*}+e_{1,2}^{*}$, i.e., we want to bound $P_{1, m}^{(1)}+P_{1, m}^{(2)}$. This has been done in Lemma 7.7. 
We next consider the second term in (8.12). We have

$$
\begin{aligned}
\left|e_{1,2}-e_{1,1}\right\rangle & =\left|1, q_{1,2}, q_{1,2}^{2}, q_{1,2}^{3}\right\rangle-\left|1, q_{1,1}, q_{1,1}^{2}, q_{1,1}^{3}\right\rangle \\
& =\left(q_{1,2}-q_{1,1}\right)\left|0,1, q_{1,1}+q_{1,2}, q_{1,1}^{2}+q_{1,1} q_{1,2}+q_{1,2}^{2}\right\rangle .
\end{aligned}
$$

We now use Eq. (7.52), the decomposition above, and

$$
F_{1, m}^{s}=\sum_{j=1}^{3} P_{1, m}^{(j)} q_{1, j}^{m} N_{1} \delta_{m, 3} .
$$

But we have seen that for $m=3$,

$$
\left|P_{1, m}^{(1)} q_{1.1}^{m}+P_{1, m}^{(2)} q_{1,2}^{m}\right| \leqq \mathbf{O}\left(\eta^{5 / 2}\right)+\mathbf{O}(1),
$$

and

$$
\left|P_{1, m}^{(3)} q_{1,3}^{m}\right| \leqq \mathbf{O}(1)
$$

Therefore

$$
\left|F_{1}^{s}\right|_{1, *} \leqq \mathbf{O}(1) \eta^{-2+\rho_{1}}\|\mathbf{b}\|_{\mathbf{B}}^{3}
$$

Thus we see that if

$$
\|\mathbf{b}\|_{\mathbf{B}} \leqq \mathbf{O}\left(\eta^{2}\right),
$$

the constant $D$ in Eq. (8.7) can be chosen $\mathbf{O}\left(\eta^{-2}\right)$. Thus we have shown the

Proposition 8.2. The nonlinearity of Eq. (4.10) is bounded by

$$
\left\|F^{s}\right\|_{\mathbf{B}} \leqq \mathbf{O}\left(\eta^{-2}\right)\|\mathbf{b}\|_{\mathbf{B}}^{3} \text {. }
$$

An analogous bound holds for $F^{u}$. Furthermore $F$ is differentiable in $\mathbf{B}$ and the derivatives satisfy similar bounds.

The last assertion follows by polarization. In view of Proposition 8.2 we see that for the front problem at $+\infty$, Theorem 8.1 holds with an $r$ of order $\mathbf{O}\left(\eta^{10}\right)$, and with a $\Phi$ which is bounded, together with its derivatives, by $\mathbf{O}\left(\eta^{-4}\right)$.

\section{Properties of the Linear Operator in the Main Sector}

In this section, we start with the analysis of the differential equation for the main sector. As we have seen in Eq. (4.19), it is of the form

$$
\begin{aligned}
& -\eta^{2} z^{\prime v}-4 i \eta \omega z^{\prime \prime \prime}-\left(2-6 \omega^{2}\right) z^{\prime \prime}+\left(c-4 i\left(1-\omega^{2}\right) \omega / \eta\right) z^{\prime} \\
& +\left(1-\left(1-\omega^{2}\right)^{2} / \eta^{2}\right) z-z|z|^{2}=h
\end{aligned}
$$

where $c>4, c-4=\mathbf{O}(\eta), \eta>0$ and $\eta=\varepsilon^{1 / 2}$, and we impose the boundary conditions

$$
z(+\infty)=0, \quad z(-\infty)=1 .
$$

We shall describe later the nature of the inhomogeneity $h$; in any event, $h$, when restricted to the interval $(-\infty, X]$, is in the space $\mathbf{H}_{\alpha, X}$. We view $z$ as a perturbation of the solution $l=l_{c}>0$ of the second order equation

$$
4 l^{\prime \prime}+c l^{\prime}+l-l^{3}=0 .
$$


The existence of such solutions, and a detailed analysis of their properties have been discussed in Sect. 5. We shall make extensive use of these results. We decompose $z$ into its real and imaginary part, and we write

$$
z=l+a+i b .
$$

This leads to a system of two coupled real fourth order equations in $a$ and $b$, and we view this system as a perturbation of the corresponding second order system. It is of the form

$$
\begin{aligned}
4 a^{\prime \prime}+c a^{\prime}+a-3 a l^{2} & =k_{1}+\operatorname{Re} h \\
4 b^{\prime \prime}+c b^{\prime}+b-b l^{2} & =k_{2}+\operatorname{Im} h,
\end{aligned}
$$

where

$$
\begin{aligned}
k_{1}= & \eta^{2}\left(l^{w}+a^{v v}\right)-4 \eta \omega \mathrm{b}^{\prime \prime \prime}+6\left(1-\omega^{2}\right)\left(l^{\prime \prime}+a^{\prime \prime}\right)-4 \frac{1-\omega^{2}}{\eta} \omega\left(l^{\prime}+a^{\prime}\right) \\
& +\frac{\left(1-\omega^{2}\right)^{2}}{\eta^{2}}(l+a)+3 a^{2} l+a^{3}+a b^{2}+l b^{2} \\
k_{2}= & \eta^{2} b^{w}+4 \eta \omega\left(l^{\prime \prime \prime}+a^{\prime \prime \prime}\right)+6\left(1-\omega^{2}\right) b^{\prime \prime}-4 \frac{1-\omega^{2}}{\eta} b^{\prime} \\
& +\frac{\left(1-\omega^{2}\right)^{2}}{\eta^{2}} b+2 a b l+a^{2} b-b^{3} .
\end{aligned}
$$

Our final aim is to show the existence of a solution to Eq. (9.1) (or, equivalently, Eq. (9.6)) by an application of the contraction mapping principle. With this aim in mind we study first the linear operator $L$ on the left-hand side of $(9.6)$, where $L$ is of the form

$$
L\left(\begin{array}{l}
a \\
b
\end{array}\right)=\left(\begin{array}{c}
4 a^{\prime \prime}+c a^{\prime}+a-3 a l^{2} \\
4 b^{\prime \prime}+c b^{\prime}+b-b l^{2}
\end{array}\right)=\left(\begin{array}{c}
L_{1} a \\
L_{2} b
\end{array}\right) .
$$

Clearly, $L$ is diagonal and hence we can study the inverse of $L$ in $a$ and $b$ separately. In other words: The original problem is a perturbation of two real decoupled second order equations.

The function space we consider controls the decay of the functions at $+\infty$ and $-\infty$. In Eq. (5.45), we have defined $\beta$ as the rate of decay of $l^{\prime}$ at $-\infty$. We now choose $\alpha<\beta$. We also choose a (large) positive constant $X$,

$$
X=C \log \left(\eta^{-1}\right)
$$

with $C$ sufficiently large. The space on which we study $L^{-1}$ is now $\mathbf{H}_{\alpha, X}$. It was defined in Sect. 6, but we recall the corresponding norm: We defined

$$
m(x)=e^{-\alpha x}+\frac{e^{x / 2}}{1+|x|} .
$$

The norm of $f, f:(-\infty, X] \rightarrow \mathbb{C}$ is given by

$$
\|f\|_{\mathbf{H}_{\alpha, X}}=\sup _{x \in(-\infty, X]}|f(x)| m(x) .
$$


Proposition 9.1. For $X$ as defined in (9.8) and for sufficiently small $\eta>0$, the maps $L_{1}^{-1}$ and $L_{2}^{-1}$ are bounded operators from $\mathbf{H}_{\alpha, X}$ to itself. Their norms are bounded by $\left(\log \left(\eta^{-1}\right)\right)^{5}$.

Remark 9.2. Similar estimates show that for $g=l^{\prime} / l$ or $g=l^{\prime \prime} / l^{\prime}$, one has the bounds

$$
\left\|\left(\partial_{x}-g\right)^{-1} f\right\|_{\mathbf{H}_{\alpha, X}} \leqq|\log \eta|^{5}\|f\|_{\mathbf{H}_{\alpha, X}} .
$$

Proof of Proposition 9.1. We consider first the operator $L_{2}$. Its inversion is based on the observation that because $l$ solves (9.3), we have, for any twice differentiable function $g$,

$$
L_{2} g=\left(4 \partial_{x}^{2}+c \partial_{x}+1-l^{2}\right) g=4\left(\partial_{x}+\frac{l^{\prime}}{l}+\frac{c}{4}\right) \cdot\left(\partial_{x}-\frac{l^{\prime}}{l}\right) g .
$$

(In fact, the second term equals

$$
\left.4 \partial_{x}^{2} g+c \partial_{x} g+4\left(-\frac{l^{\prime \prime}}{l}+\left(\frac{l^{\prime}}{l}\right)^{2}-\left(\frac{l^{\prime}}{l}\right)^{2}-\frac{c l^{\prime}}{4 l}\right) g .\right)
$$

We now note that the equation

$$
y^{\prime}-(\log u)^{\prime} y=v
$$

has the solution

$$
y(x)=u(x) \cdot\left(\text { const }+\int_{x_{0}}^{x} d s v(s) / u(s)\right)
$$

Since we have

$$
L_{2}=4\left(\partial_{x}+\left(\log l(x) e^{c x / 4}\right)^{\prime}\right)\left(\partial_{x}-(\log l(x))^{\prime}\right)
$$

we find that $L_{2}^{-1} f$ is of the form

$$
L_{2}^{-1} f(x)=\frac{l(x)}{4} \int_{-\infty}^{x} d s \frac{e^{-c s / 4}}{l(s)^{2}} \int_{-\infty}^{s} d t l(t) e^{c t / 4} f(t)
$$

Our choice of boundary condition will become clear later. Since $l^{\prime}$ solves the equation

$$
4 l^{\prime \prime \prime}+c l^{\prime \prime}+l^{\prime}-3 l^{\prime} l^{2}=0
$$

we have for the case of $L_{1}$ :

$$
L_{1} g=4\left(\partial_{x}+\frac{l^{\prime \prime}}{l^{\prime}}+\frac{c}{4}\right) \cdot\left(\partial_{x}-\frac{l^{\prime \prime}}{l^{\prime}}\right) g
$$

Therefore, we find that $L_{1}^{-1} f$ is of the form

$$
L_{1}^{-1} f(x)=\frac{l^{\prime}(x)}{4} \int_{-\infty}^{x} d s \frac{e^{-c s / 4}}{l^{\prime}(s)^{2}} \int_{-\infty}^{s} d t l^{\prime}(t) e^{c t / 4} f(t)
$$

We now start to bound the integrals in (9.14) and (9.17). By linearity, we may assume $\|f\|_{\mathbf{H}_{\alpha, X}}=1$. By our definition (9.8) of $X$, and the restriction (3.5) on $c$, we have $X<$ $(c-4)^{-1 / 2}$. Therefore, (5.24) holds. In fact, this bound fails for $X=\infty$, and this is the 
reason for our introduction of $X$. For $s \leqq 0$, we find, using the definition of $m$,

$$
\left|\int_{-\infty}^{s} d t l(t) e^{c t / 4} f(t)\right| \leqq \mathbf{O}(1) \int_{-\infty}^{s} d t e^{c t / 4} e^{\alpha t}\|f\|_{\mathbf{H}_{\alpha, X}} \leqq \mathbf{O}\left(e^{(\alpha+c / 4) s}\right) .
$$

When $X \geqq s \geqq 0$, we see from (5.24) that

$$
\left|\int_{0}^{s} d t l(t) e^{c t / 4} f(t)\right| \leqq \mathbf{O}(1) \int_{0}^{s} d t e^{c t / 4}\left(e^{-t / 2}(t+1)\right)^{2} \leqq \mathbf{O}(1)(s+1)^{3} .
$$

We next assume $x \leqq 0$. Then we have $1>l(x) \geqq 1 / 2$ and hence, by the definition (9.14), and by (9.18),

$$
\left|m(x) L_{2}^{-1} f(x)\right| \leqq \mathbf{O}(1) e^{-\alpha x} \int_{-\infty}^{x} d s e^{-c s / 4} e^{(\alpha+c / 4) s} \leqq \mathbf{O}(1) .
$$

When $X \geqq x \geqq 0$, we find, using Lemma 5.2 and Lemma 5.3, and the bounds (9.18), (9.19),

$$
\begin{aligned}
\left|m(x) L_{2}^{-1} f(x)\right| & \leqq \mathbf{O}(1) m(x) l(x)\left(1+\int_{0}^{x} d s e^{-c s / 4} e^{2 c s / 8}(s+1)^{3}\right) \\
& \leqq \mathbf{O}\left(X^{4}\right) \leqq\left(\log \left(\eta^{-1}\right)\right)^{5} .
\end{aligned}
$$

Thus we have obtained the asserted bound on $L_{2}^{-1}$.

The case of $L_{1}^{-1}$ is similar. For $s \leqq 0$, we have, by Lemma 5.7,

$$
\begin{aligned}
\left|\int_{-\infty}^{s} d t l^{\prime}(t) e^{c t / 4} f(t)\right| & \leqq \mathbf{O}(1) \int_{-\infty}^{s} d t e^{\beta t} e^{c t / 4} e^{\alpha t}\|f\|_{\mathbf{H}_{\alpha, X}} \\
& \leqq \mathbf{O}(1) e^{(\beta+c / 4+\alpha) s} .
\end{aligned}
$$

For $s \geqq 0$, we have, by Lemma 5.4 ,

$$
\begin{aligned}
\left|\int_{0}^{s} d t l^{\prime}(t) e^{c t / 4} f(t)\right| & \leqq \mathbf{O}(1) \int_{0}^{s} d t e^{\left(\gamma_{+}+c / 4-1 / 2\right) t}(t+1) \\
& \leqq \mathbf{O}(1)(s+1)^{2} e^{(\gamma++c / 4-1 / 2) s}
\end{aligned}
$$

Next, we assume $x \leqq 0$ and bound the second integral in (9.17). Using Lemma 5.7, we find

$$
\left|m(x) L_{1}^{-1} f(x)\right| \leqq \mathbf{O}(1) e^{-(\alpha-\beta) x} \int_{-\infty}^{x} d s e^{-c s / 4} e^{-2 \beta s} e^{(\beta+c / 4+\alpha) s} \leqq \mathbf{O}(1) .
$$

When $x \geqq 0$, we use Lemma 5.5, and we find

$$
\begin{gathered}
\left|m(x) L_{1}^{-1} f(x)\right| \\
\leqq \mathbf{O}(1) e^{\left(\gamma_{+}+1 / 2\right) x}\left(1+\int_{0}^{x} d s e^{-c s / 4} e^{-2 \gamma_{+} s} e^{\left(\gamma_{+}+c / 4-1 / 2\right) s}(s+1)^{2}\right) \\
\leqq \mathbf{O}(1) e^{\left(c^{2}-16\right)^{1 / 2} x}(x+1)^{3} \leqq \mathbf{O}\left(e^{\mathbf{O}(\eta \log \eta)}|\log \eta|^{3}\right) .
\end{gathered}
$$

This completes the proof of Proposition 9.1. The proof of Remark 9.2 is left to the reader. 
We now come back to our main problem. Equation (9.5) can be written as

$$
\begin{aligned}
& a=L_{1}^{-1}\left(k_{1}+\operatorname{Re} h\right), \\
& b=L_{2}^{-1}\left(k_{2}+\operatorname{Im} h\right) .
\end{aligned}
$$

We now decompose

$$
\begin{aligned}
& k_{1}=k_{11}+k_{12}, \quad \text { with } k_{11}=\eta^{2} a^{i v}-4 \eta \omega b^{\prime \prime \prime}, \\
& k_{2}=k_{21}+k_{22}, \quad \text { with } k_{21}=\eta^{2} b^{i v}+4 \eta \omega a^{\prime \prime \prime} .
\end{aligned}
$$

We want to write $L_{j}^{-1} k_{j 1}$ as a differential operator acting on $(a, b)$ plus a 'small' remainder.

Proposition 9.3. There are bounded (linear) operators $\mathbf{O}_{i j}, i=1,2, j=3,4$, on $\mathbf{H}_{\alpha, X}$, with a norm bounded by $|\log \eta|^{5}$, such that for all $f \in \mathbf{H}_{\alpha, X}$ which are four times differentiable, one has

$$
\begin{aligned}
& 4 L_{1}^{-1} \partial^{4} f=f^{\prime \prime}-\frac{c}{4} f^{\prime}+\mathbf{O}_{14} f, \\
& 4 L_{2}^{-1} \partial^{4} f=f^{\prime \prime}-\frac{c}{4} f^{\prime}+\mathbf{O}_{24} f, \\
& 4 L_{1}^{-1} \partial^{3} f=f^{\prime}-\frac{c}{4} f+\left(\partial_{x}-l^{\prime \prime} / l^{\prime}\right)^{-1} \mathbf{O}_{13} f, \\
& 4 L_{2}^{-1} \partial^{3} f=f^{\prime}-\frac{c}{4} f+\left(\partial_{x}-l^{\prime} / l^{-1} \mathbf{O}_{23} f .\right.
\end{aligned}
$$

Proof. We consider first $D^{-1}$, where $D=\partial_{x}-g$ for some function $g$. We may write $\partial_{x}=D+g$ and expand. One has then the following identities.

$$
\begin{aligned}
D^{-1} \partial_{x} & =D^{-1}(D+g)=\mathrm{id}+D^{-1} g, \\
D^{-1} \partial_{x}^{2} & =D^{-1}(D+g)^{2}=D^{-1} D(D+g)+D^{-1} g(D+g) \\
& =\partial_{x}+g-D^{-1} g^{\prime}+D^{-1} g^{2},
\end{aligned}
$$

(we have used $g D=D g-g^{\prime}$ ). Similarly,

$$
\begin{aligned}
\partial_{x}^{3} & =D(D+g)^{2}+g D(D+g)+g^{2} D+g^{3} \\
& =D \partial_{x}^{2}+D g \partial_{x}-g^{\prime}(D+g)+D g^{2}-2 g^{\prime} g+g^{3} \\
& =D \partial_{x}^{2}+D g \partial_{x}-D g^{\prime}+g^{\prime \prime}-g^{\prime} g+D g^{2}-2 g^{\prime} g+g^{3},
\end{aligned}
$$

so that

$$
D^{-1} \partial_{x}^{3}=\partial_{x}^{2}+g \partial_{x}-g^{\prime}+g^{2}+D^{-1}\left\{g^{\prime \prime}-3 g^{\prime} g+g^{3}\right\} .
$$

Finally,

$$
\begin{aligned}
\partial_{x}^{4} & =D(D+g)^{3}+g D(D+g)^{2}+g^{2} D(D+g)+g^{3} D+g^{4} \\
& =D \partial_{x}^{3}+D g \partial_{x}^{2}-g^{\prime}(D+g)^{2}+D g^{2} \partial_{x}-2 g^{\prime} g(D+g)+D g^{3}-3 g^{\prime} g^{2}+g^{4} .
\end{aligned}
$$


We have the two identities

$$
\begin{aligned}
-g^{\prime}(D+g)^{2} & =-g^{\prime} D(D+g)-g^{\prime} g(D+g) \\
& =-D g^{\prime} \partial_{x}+g^{\prime \prime}(D+g)-D g^{\prime} g+\left(g^{\prime} g\right)^{\prime}-g^{\prime} g^{2} \\
& =-D g^{\prime} \partial_{x}+D g^{\prime \prime}-g^{\prime \prime \prime}+g^{\prime \prime} g-D g^{\prime} g+g^{\prime \prime} g+\left(g^{\prime}\right)^{2}-g^{\prime} g^{2}
\end{aligned}
$$

and

$$
-2 g g^{\prime}(D+g)=-2 D g^{\prime} g+2\left(g^{\prime} g\right)^{\prime}-2 g^{\prime} g^{2}
$$

Collecting these terms, we get

$$
\begin{aligned}
D^{-1} \partial_{x}^{4}= & \partial_{x}^{3}+g \partial_{x}^{2}+g^{2} \partial_{x}+g^{3}-g^{\prime} \partial_{x}+g^{\prime \prime}-3 g^{\prime} g \\
& +D^{-1}\left\{g^{4}-6 g^{\prime} g^{2}-g^{\prime \prime \prime}+4 g^{\prime \prime} g+3\left(g^{\prime}\right)^{2}\right\} \\
= & \partial_{x}^{3}+\partial_{x}^{2} g-3 \partial_{x} g^{\prime}+\partial_{x} g^{2}+g^{3}-5 g^{\prime} g+3 g^{\prime \prime} \\
& +D^{-1}\left\{g^{4}-6 g^{\prime} g^{2}-g^{\prime \prime \prime}+4 g^{\prime \prime} g+3\left(g^{\prime}\right)^{2}\right\}
\end{aligned}
$$

Going back to our original problem, we have

$$
L_{1}=4\left(\partial_{x}-g_{1}\right)\left(\partial_{x}-g_{2}\right)
$$

with $g_{2}=l^{\prime \prime} / l^{\prime}, g_{1}=-l^{\prime \prime} / l^{\prime}-c / 4$. We thus find

$$
\begin{aligned}
4 L_{1}^{-1} a^{v v}= & \left(\partial_{x}-g_{2}\right)^{-1}\left(\partial_{x}-g_{1}\right)^{-1} \partial_{x}^{4} a \\
= & \left(\partial_{x}-g_{2}\right)^{-1}\left\{\partial_{x}^{3}+\partial_{x}^{2} g_{1}-3 \partial_{x} g_{1}+\partial_{x} g_{1}^{2}+g_{1}^{3}-5 g_{1}^{\prime} g_{1}+3 g_{1}^{\prime \prime}\right\} a \\
& +4 L_{1}^{-1}\left\{g_{1}^{4}-6 g_{1}^{\prime} g_{1}^{2}-g_{1}^{\prime \prime \prime}+4 g_{1}^{\prime \prime} g_{1}+3\left(g_{1}^{\prime}\right)^{2}\right\} .
\end{aligned}
$$

Using the techniques of Sect. 5, cf. in particular the vicinity of Eq. (5.41), we see that $g_{1}, g_{2}$, and their derivatives are bounded (by $\mathbf{O}(1)$ ). Thus the last bracket above is bounded and we find

$$
\left\|4 L_{1}^{-1}\{\cdots\} f\right\|_{\mathbf{H}_{\alpha, X}} \leqq \mathbf{O}(1)|\log \eta|^{5}\|f\|_{\mathbf{H}_{\alpha, X}}
$$

by Proposition 9.1. Reapplying the identities for $\partial_{x}^{k}$, we get

$$
\begin{aligned}
4 L_{1}^{-1} a^{\iota v}= & \left(\partial_{x}^{2}+g_{2} \partial_{x}-g_{2}^{\prime}+g_{2}^{2}\right) a+\left(\partial_{x}-g_{2}\right)^{-1}\left\{g_{1}^{\prime \prime}-3 g_{1}^{\prime} g_{1}+g_{1}^{3}\right\} a \\
& +\partial_{x} g_{1} a+g_{1} g_{2} a+\mathbf{O}_{3} a \\
= & a^{\prime \prime}+\left(g_{1}+g_{2}\right) a^{\prime}+\left(g_{2}^{2}+g_{1}^{\prime}-g_{2}^{\prime}+g_{1} g_{2}\right) a+\mathbf{O}_{4} a \\
= & a^{\prime \prime}-\frac{c}{4} a^{\prime}+\mathbf{O}_{5} a .
\end{aligned}
$$

Here, and below, all $\mathbf{O}_{j}$ denote bounded (linear) operators on $\mathbf{H}_{\alpha, X}$, with norm bounded by $\mathbf{O}\left(|\log \eta|^{5}\right)$, as is seen by applying Proposition 9.1 and Remark 9.2.

In the case of the third derivative, we need a somewhat more detailed analysis of the "error term." We have, using Eq. (9.34),

$$
\begin{aligned}
4 L_{1}^{-1} \partial_{x}^{3}= & \left(\partial_{x}-g_{2}\right)^{-1}\left\{\partial_{x}^{2}+\partial_{x} g_{1}-2 g_{1}^{\prime}+g_{1}^{2}+\left(\partial_{x}-g_{1}\right)^{-1}\left\{g_{1}^{\prime \prime}-3 g_{1}^{\prime} g_{1}+g_{1}^{3}\right\}\right\} \\
= & \partial_{x}+g_{1}+g_{2}+\left(\partial_{x}-g_{2}\right)^{-1}\left\{-g_{2}^{\prime}+g_{2}^{2}+g_{1} g_{2}-2 g_{1}^{\prime}+g_{1}^{2}\right\} \\
& +\left(\partial_{x}-g_{2}\right)^{-1}\left(\partial_{x}-g_{1}\right)^{-1}\left\{g_{1}^{\prime \prime}-3 g_{1}^{\prime} g_{1}+g_{1}^{3}\right\} .
\end{aligned}
$$


Thus, we get

$$
4 L_{1}^{-1} b^{\prime \prime \prime}=b^{\prime}-\frac{c}{4} b+\left(\partial_{x}-l^{\prime \prime} / l^{\prime}\right)^{-1} \mathbf{O}_{6} b
$$

The other cases are now analogous:

$$
\begin{aligned}
& 4 L_{2}^{-1} b^{\imath v}=b^{\prime \prime}-\frac{c}{4} b^{\prime}+\mathbf{O}_{7} \mathbf{b}, \\
& 4 L_{2}^{-1} a^{\prime \prime \prime}=a^{\prime}-\frac{c}{4} a+\left(\partial_{x}-l^{\prime} / l\right)^{-1} \mathbf{O}_{8} a .
\end{aligned}
$$

The proof of Proposition 9.3 is complete.

The expressions $a_{j}-L_{j}^{-1} k_{j 1}, j=1,2$ (with $a_{1}=a, a_{2}=b$ ) can now be written as

$$
\begin{aligned}
& a-L_{1}^{-1} k_{11}=a-\frac{1}{2} \eta^{2} a^{\prime \prime}+\frac{c}{16} \eta^{2} a^{\prime}+\eta \omega b^{\prime}-\eta \frac{c}{4} b-\mathbf{O}_{1}(a, b), \\
& b-L_{2}^{-1} k_{21}=b-\frac{1}{4} \eta^{2} b^{\prime \prime}+\frac{c}{16} \eta^{2} b^{\prime}-\eta \omega a^{\prime}+\eta \frac{c}{4} a-\mathbf{O}_{2}(a, b) .
\end{aligned}
$$

Here $\mathbf{O}_{i}, i=1,2$ is of the form

$$
\mathbf{O}_{i}=\eta\left(\partial_{x}-g\right)^{-1} \mathbf{O}_{i 1}+\eta^{2} \mathbf{O}_{i 2},
$$

where the $\mathbf{O}_{i j}$ are bounded linear operators from $\mathbf{H}_{\alpha, X} \oplus \mathbf{H}_{\alpha, X}$ to $\mathbf{H}_{\alpha, X}$, with norm bounded by $\mathbf{O}\left(|\log \eta|^{5}\right)$, and $g=l^{\prime \prime} / l^{\prime}$ or $g=l^{\prime} / l$.

We study next the operator $\mathbf{M}$ defined by the right-hand side of Eq. (9.44) (without the terms $\mathbf{O}_{j}$ ) in Fourier space, using techniques analogous to those of Sect. 7. We see that the correspondence $\partial_{x} \rightarrow i q$ leads to the matrix (with constant coefficients)

$$
\left[\begin{array}{cc}
1+\frac{\eta^{2} q^{2}}{4}+\frac{i \eta^{2} q c}{16} & i \eta \omega q-\frac{\eta c}{4} \\
-i \eta \omega q+\frac{\eta c}{4} & 1+\frac{\eta^{2} q^{2}}{4}+\frac{i \eta^{2} q c}{16}
\end{array}\right]
$$

The characteristic polynomial of this matrix, when expressed in the variable $p=\eta q / 2$, is of the form

$$
\Delta(p)=\left(1-p^{2}+i \eta p \frac{c}{8}\right)^{2}+\left(\frac{\eta c}{4}-2 i \omega p\right)^{2} .
$$

For $\eta=0$, this polynomial has the roots $p= \pm 1$, and one can check that for small $\eta$, the four roots are of the form

$$
\left.\begin{array}{l}
p_{1,2}=1 \pm \eta^{1 / 2}\left(\frac{i c}{8}\right)^{1 / 2}+\mathbf{O}(\eta) \\
p_{3,4}=-1 \pm \eta^{1 / 2} i\left(\frac{c}{8}\right)^{1 / 2}+\mathbf{O}(\eta)
\end{array}\right\}
$$


We have used $\omega^{2}-1=\mathbf{O}\left(\eta^{2}\right)$. We also define $q_{j}=2 p_{j} / \eta$. The inverse of the matrix (9.46) is of the form $\mathbf{R}(q) / \Delta(\eta q)$, where $\mathbf{R}$ is given by

$$
\left[\begin{array}{cc}
1+\frac{\eta^{2} q^{2}}{4}+\frac{i \eta^{2} q c}{16} & -i \eta \omega q+\frac{\eta c}{4} \\
i \eta \omega q-\frac{\eta c}{4} & 1+\frac{\eta^{2} q^{2}}{4}+\frac{i \eta^{2} q c}{16}
\end{array}\right]
$$

If we denote by $r$ the pair of functions $r_{1}, r_{2}$, we can define $\mathbf{M}^{-1}$ as follows:

$$
\begin{aligned}
\left(\mathbf{M}^{-1} r\right)(x)= & \sum_{j=1,3} \int_{-\infty}^{x} d y \frac{\mathbf{R}\left(q_{j}\right) r(y)}{\Delta^{\prime}\left(\eta q_{j} / 2\right) \eta / 2} e^{i q_{j}(x-y)} \\
& -\sum_{j=2,4} \int_{x}^{X} d y \frac{\mathbf{R}\left(q_{j}\right) r(y)}{\Delta^{\prime}\left(\eta q_{j} / 2\right) \eta / 2} e^{i q_{j}(x-y)} .
\end{aligned}
$$

Proposition 9.4. The operator $\mathbf{M}^{-1}$ is defined as a bounded linear map from $\mathbf{H}_{\alpha, X} \oplus \mathbf{H}_{\alpha, X}$ to itself. Its norm is bounded by $\mathbf{O}\left(\eta^{-1}\right)$. If $g=l^{\prime \prime} / l^{\prime}$ or $g=l^{\prime} / l$, then the norm of $\mathbf{M}^{-1}\left(\partial_{x}-g\right)^{-1}$ is bounded by $\mathbf{O}\left(\eta^{-1 / 2}\right)$.

Proof. We first observe that $\mathbf{R}\left(q_{j}\right)$ is $\mathbf{O}(1)$. Given the identities (9.48), we see that

$$
\frac{1}{\left|\Delta^{\prime}\left(\eta q_{j} / 2\right) \eta / 2\right|} \leqq \mathbf{O}\left(\eta^{-3 / 2}\right)
$$

To bound the norm of the operator $\mathbf{M}^{-1}$ from $\mathbf{H}_{\alpha, X}$ to itself, (in fact, from $\mathbf{H}_{\alpha, X} \oplus \mathbf{H}_{\alpha, X}$ to itself, but we use the same notation for the norm on the direct sum), we use again the estimates from Sect. 6. Note that $\left|\operatorname{Im} q_{j}\right|=\mathbf{O}\left(\eta^{-1 / 2}\right) \gg 1$. Therefore, we find that

$$
\left\|\mathbf{M}^{-1} r\right\|_{\mathbf{H}_{\alpha, X}} \leqq \mathbf{O}\left(\eta^{-1}\right)\|r\|_{\mathbf{H}_{\alpha, X}},
$$

i.e., the inverse of $\mathbf{M}$ is bounded by $\mathbf{O}\left(\eta^{-1}\right)$.

We next bound $\mathbf{M}^{-1}\left(\partial_{x}-g\right)^{-1}$, with $g$ as in the statement of the proposition. We consider first $\mathbf{M}^{-1}\left(\partial_{x}+1\right)^{-1}$. Setting $s=\left(\partial_{x}+1\right)^{-1} r$, we see that $\mathbf{M}^{-1} s$ leads to integral expressions as in (9.50), which can be integrated by parts. E.g. for $j=2,4$, we get expressions of the form

$$
\frac{1}{i q_{j}} \int_{x}^{X} d y \frac{\mathbf{R}\left(q_{j}\right) s^{\prime}(y)}{\Delta^{\prime}\left(\eta q_{j} / 2\right) \eta / 2} e^{i q_{j}(x-y)},
$$

plus boundary terms. When taking the sup (over $x$ ) of these expressions (after multiplying by the weight factor $m(x)$ ), we find that the boundary term at $X$ is critical. Using the techniques leading to Lemma 7.4, we see that

$$
\left\|\mathbf{M}^{-1}\left(\partial_{x}+1\right)^{-1} r\right\|_{\mathbf{H}_{\alpha, X}} \leqq \mathbf{O}\left(\eta^{-1 / 2}\right)\left\|\partial_{x} s\right\|_{\mathbf{H}_{\alpha, X}} .
$$

We have $\left\|\partial_{x} s\right\|_{\mathbf{H}_{\alpha, X}} \leqq\|r\|_{\mathbf{H}_{\alpha, X}}+\left\|\left(\partial_{x}+1\right)^{-1} r\right\|_{\mathbf{H}_{\alpha, X}}$, and by Lemma 6.2, this is bounded by $\mathbf{O}\left(\|r\|_{\mathbf{H}_{z, X}}\right)$. Thus we have shown

$$
\left\|\mathbf{M}^{-1}\left(\partial_{x}+1\right)^{-1} f\right\|_{\mathbf{H}_{x, X}}<\mathbf{O}\left(\eta^{-1 / 2}\right)\|f\|_{\mathbf{H}_{\alpha, X}} .
$$


From this we get the inequality

$$
\begin{aligned}
\left\|\mathbf{M}^{-1}\left(\partial_{x}-g\right)^{-1} f\right\|_{\mathbf{H}_{\alpha, X}} & \leqq \mathbf{O}\left(\eta^{-1 / 2}\right)\left\|\left(\partial_{x}+1\right)\left(\partial_{x}-g\right)^{-1} f\right\|_{\mathbf{H}_{\alpha, X}} \\
& <\mathbf{O}\left(\eta^{-1 / 2}\right)\|f\|_{\mathbf{H}_{\alpha, X}},
\end{aligned}
$$

where the last inequality follows from

$$
\left(\partial_{x}+1\right)\left(\partial_{x}-g\right)^{-1}=1+(g-1)\left(\partial_{x}-g\right)^{-1}
$$

and (9.54). The Proposition 9.4 is proved.

In Sects. 12 and 13, we shall need to study the operators

$$
P_{1, m}^{(4)}\left(-i \partial_{x}\right)^{m} \mathbf{M}^{-1}, \text { for } m=0, \ldots, 3
$$

These operators exhibit strong cancellations, which come about as follows. The projection $P_{1}^{(4)}$ is associated with the operator $A_{\omega}$, while $\mathbf{M}$ is the "quotient" of $A_{\omega}$ and the second order operator $\left(L_{1}, L_{2}\right)$. Thus, the large eigenvalues of $A_{\omega}$ and of $\mathbf{M}$ almost coincide, leading to the cancellation which we study now. We have

$$
\begin{aligned}
\left(-i \partial_{x} \mathbf{M}^{-1} r\right)(x)= & \sum_{j=1,3} \int_{-\infty}^{x} d y \frac{\mathbf{U}_{j} \mathbf{R}\left(q_{j}\right) r(y)}{\Delta^{\prime}\left(\eta q_{j} / 2\right) \eta / 2} e^{i q_{j}(x-y)} \\
& -\sum_{j=2,4} \int_{x}^{x} d y \frac{\mathbf{U}_{j} \mathbf{R}\left(q_{j}\right) r(y)}{\Delta^{\prime}\left(\eta q_{j} / 2\right) \eta / 2} e^{i q_{j}(x-y)} .
\end{aligned}
$$

Here, $\mathbf{U}_{j}$ is the matrix

$$
\mathbf{U}_{j}=\left(\begin{array}{rr}
\operatorname{Re} q_{j} & -\operatorname{Im} q_{j} \\
\operatorname{Im} q_{j} & \operatorname{Re} q_{j}
\end{array}\right) .
$$

The boundary terms at $x$ have cancelled by a mechanism analogous to that of Lemma 7.3. For the second derivative, we find

$$
\begin{aligned}
\left(\left(-i \partial_{x}\right)^{2} \mathbf{M}^{-1} r\right)(x)= & \sum_{j=1,3} \int_{-\infty}^{x} d y \frac{\mathbf{U}_{j}^{2} \mathbf{R}\left(q_{j}\right) r(y)}{\Delta^{\prime}\left(\eta q_{j} / 2\right) \eta / 2} e^{i q_{j}(x-y)} \\
& -\sum_{j=2,4} \int_{x}^{x} d y \frac{\mathbf{U}_{j}^{2} \mathbf{R}\left(q_{j}\right) r(y)}{\Delta^{\prime}\left(\eta q_{j} / 2\right) \eta / 2} e^{i q_{j}(x-y)}+\sum_{j=1}^{4} \frac{\mathbf{U}_{j} \mathbf{R}\left(q_{j}\right) r(x)}{\Delta^{\prime}\left(\eta q_{j} / 2\right) \eta / 2} .
\end{aligned}
$$

Similarly, we find

$$
\begin{aligned}
\left(\left(-i \partial_{x}\right)^{3} \mathbf{M}^{-1} r\right)(x)= & \sum_{j=1,3} \int_{-\infty}^{x} d y \frac{\mathbf{U}_{j}^{3} \mathbf{R}\left(q_{j}\right) r(y)}{\Delta^{\prime}\left(\eta q_{j} / 2\right) \eta / 2} e^{i q_{j}(x-y)} \\
& -\sum_{j=2,4} \int_{x}^{X} d y \frac{\mathbf{U}_{j}^{3} \mathbf{R}\left(q_{j}\right) r(y)}{\Delta^{\prime}\left(\eta q_{j} / 2\right) \eta / 2} e^{i q_{j}(x-y)} \\
& +\sum_{j=1}^{4} \frac{\mathbf{U}_{j}^{2} \mathbf{R}\left(q_{j}\right) r(x)}{\Delta^{\prime}\left(\eta q_{j} / 2\right) \eta / 2}+\sum_{j=1}^{4} \frac{\mathbf{U}_{j} \mathbf{R}\left(q_{j}\right) \mathbf{D} r(x)}{\Delta^{\prime}\left(\eta q_{j} / 2\right) \eta / 2}
\end{aligned}
$$

The matrix $\mathbf{D}$ is given by

$$
\mathbf{D}=\left(\begin{array}{rr}
0 & \partial_{x} \\
-\partial_{x} & 0
\end{array}\right)
$$


In studying (9.55), we are faced by a product of matrices of the type of $\mathbf{U}_{j}$. To simplify notation, we write, however, all expressions in scalar form, except where the matricial cancellation will be crucial. We see from (9.56)-(9.58), that (9.55) is an integral plus a sum of boundary terms. We start by bounding the integral

$$
\sum_{j=1}^{4} \pm \int d y \frac{\left(q_{j}-q_{1,1}\right)\left(q_{j}-q_{1,2}\right)\left(q_{j-1,3}\right)}{\left(q_{1,4}-q_{1,1}\right)\left(q_{1,4}-q_{1,2}\right)\left(q_{1,4}-q_{1,3}\right)} \frac{\mathbf{R}\left(q_{j}\right) r(y)}{\Delta^{\prime}\left(\eta q_{j} / 2\right) \eta / 2} e^{i q_{j}(x-y)} .
$$

The signs and integration limits are as in (9.50). By (7.35) and (7.38), we have

$$
\begin{aligned}
& q_{1,1}, q_{1,2}=i / 2+\mathbf{O}\left(\eta^{1 / 2}\right), \\
& q_{1,3}, q_{1,4}=-2 / \eta+\mathbf{O}\left(\eta^{-1 / 2}\right) .
\end{aligned}
$$

We are lead to a matrix multiplication $\left(q_{j}-q_{1,3}\right) \mathbf{R}\left(q_{j}\right)$, expressed for legibility in the $p_{j}=\eta q_{j} / 2$,

$$
\eta^{-1 / 2}\left(\begin{array}{cc}
1+\mathbf{O}\left(\eta^{1 / 2}\right) & i p_{j}+\mathbf{O}\left(\eta^{1 / 2}\right) \\
-i p_{j}+\mathbf{O}\left(\eta^{1 / 2}\right) & 1+\mathbf{O}\left(\eta^{1 / 2}\right)
\end{array}\right)\left(\begin{array}{cc}
1+p_{j}^{2}+\mathbf{O}(\eta) & -2 i p_{j}+\mathbf{O}(\eta) \\
2 i p_{j}+\mathbf{O}(\eta) & 1+p_{j}^{2}+\mathbf{O}(\eta)
\end{array}\right)=\mathbf{O}\left(\eta^{-1 / 2}\right)
$$

Note now that the quotients in (9.59) lead to the bounds

$$
\frac{\left(q_{j}-q_{1,1}\right)\left(q_{j}-q_{1,2}\right)}{\left(q_{1,4}-q_{1,1}\right)\left(q_{1,4}-q_{1,2}\right)\left(q_{1,4}-q_{1,3}\right)}=\mathbf{O}\left(\eta^{1 / 2}\right) \text {. }
$$

Combining this with (9.60) and (9.51) we see that the norm of (9.59) in $\mathbf{H}_{\alpha, X}$ is bounded by

$$
\mathbf{O}\left(\eta^{-1}\right)\|r\|_{\mathbf{H}_{\alpha, X}}
$$

We next study the boundary terms.

Lemma 9.5. We have the bounds

$$
\sum_{j=1}^{4} \frac{\mathbf{U}_{j} \mathbf{R}\left(q_{j}\right)}{\Delta^{\prime}\left(\eta q_{j} / 2\right) \eta / 2}=\mathbf{O}\left(\eta^{-2}\right), \quad \sum_{j=1}^{4} \frac{\mathbf{U}_{j}^{2} \mathbf{R}\left(q_{j}\right)}{\Delta^{\prime}\left(\eta q_{j} / 2\right) \eta / 2}=\mathbf{O}\left(\eta^{-3}\right) \text {. }
$$

Proof. The proof is similar to the proof of Lemma 7.3 and is left to the reader.

We now summarize our results, by multiplying the various estimates. We have already summed the integrated terms. The boundary terms for $m=2$ contribute

$$
\mathbf{O}(1) \eta^{5 / 2} \eta^{-1} \eta^{-2}\|r\|_{\mathbf{H}_{\alpha, X}}=\mathbf{O}\left(\eta^{-1 / 2}\right)\|r\|_{\mathbf{H}_{\alpha, X}},
$$

and for $m=3$, we get

$\mathbf{O}(1) \eta^{5 / 2}\left(\eta^{-3}\|r\|_{\mathbf{H}_{\alpha, X}}+\eta^{-2}\|\mathbf{D} r\|_{\mathbf{H}_{\alpha, X}}\right)=\mathbf{O}\left(\eta^{-1 / 2}\right)\|r\|_{\mathbf{H}_{\alpha, X}}+\mathbf{O}\left(\eta^{1 / 2}\right)\|\mathbf{D} r\|_{\mathbf{H}_{\alpha, X}}$.

We can improve the above estimates, as in (9.53).

Proposition 9.6. One has the bound

$$
\left\|\sum_{m=0}^{3} P_{1, m}^{(4)}\left(-i \partial_{x}\right)^{m} \mathbf{M}^{-1}(1+\mathbf{D})^{-1} s\right\|_{\mathbf{H}_{\alpha, X}} \leqq \mathbf{O}\left(\eta^{-1 / 2}\right)\|s\|_{\mathbf{H}_{\alpha, X}} .
$$




\section{Perturbation Theory}

In Sect. 9, we solved equations of the form

$$
L\left(\begin{array}{l}
a \\
b
\end{array}\right)=h .
$$

We now show how to obtain an approximate solution to any given order $N$ of Eq. (9.5) with $h=0$, which we now write as

$$
L u=K(u)+v .
$$

Here, the unknown $u$ has two components $u_{1}, u_{2}$. Furthermore,

$$
\begin{aligned}
L\left(\begin{array}{l}
u_{1} \\
u_{2}
\end{array}\right) & =\left(\begin{array}{l}
4 u_{1}^{\prime \prime}+c u_{1}^{\prime}+u_{1}-3 u_{1} l^{2} \\
4 u_{2}^{\prime \prime}+c u_{2}^{\prime}+u_{2}-u_{2} l^{2}
\end{array}\right), \\
K\left(\begin{array}{l}
u_{1} \\
u_{2}
\end{array}\right) & =\left(\begin{array}{l}
K_{1} \\
K_{2}
\end{array}\right)
\end{aligned}
$$

with

$$
\begin{aligned}
K_{1}\left(u_{1}, u_{2}\right)= & \eta^{2} u_{1}^{\imath v}-4 \eta \omega u_{2}^{\prime \prime \prime}+6\left(1-\omega^{2}\right) u_{1}^{\prime \prime}-4 \frac{1-\omega^{2}}{\eta} \omega u_{1}^{\prime} \\
& +\frac{\left(1-\omega^{2}\right)^{2}}{\eta^{2}} u_{1}+3 u_{1}^{2} l+u_{1}^{3}+u_{1} u_{2}^{2}+u_{2}^{2} l \\
K_{2}\left(u_{1}, u_{2}\right)= & \eta^{2} u_{2}^{i v}+4 \eta \omega u_{1}^{\prime \prime \prime}+6\left(1-\omega^{2}\right) u_{2}^{\prime \prime}-4 \frac{1-\omega^{2}}{\eta} u_{2}^{\prime} \\
& +\frac{\left(1-\omega^{2}\right)^{2}}{\eta^{2}} u_{2}+2 u_{1} u_{2} l+u_{1}^{2} u_{2}-u_{2}^{3} .
\end{aligned}
$$

Finally, we have $v=\left(v_{1}, v_{2}\right)$ with

$$
\begin{aligned}
& v_{1}=\eta^{2} l^{v}+6\left(1-\omega^{2}\right) l^{\prime \prime}-4 \frac{1-\omega^{2}}{\eta} \omega l^{\prime}+\frac{\left(1-\omega^{2}\right)^{2}}{\eta^{2}} l, \\
& v_{2}=4 \eta \omega l^{\prime \prime \prime} .
\end{aligned}
$$

We can solve iteratively Eq. (10.2) by using the results of Sect. 9 and the bounds on $l$ from Sect. 5. We define

$$
u_{0}=0, \quad u_{j}=L^{-1} K\left(u_{j-1}\right)+L^{-1} v .
$$

We fix, once and for all, an integer $N$ (the degree of approximation). The constants below will depend on $N$ but not on $\eta$.

Proposition 10.1. One has the bound

$$
\left\|L u_{N}-K\left(u_{N}\right)-v\right\|_{\mathbf{H}_{\alpha, X}} \leqq \mathbf{O}(1) \eta^{N}|\log \eta|^{5 N} .
$$

Proof. We consider first the operator $L^{-1}$. By Proposition 9.1, we have

$$
\left\|L^{-1} u\right\|_{\mathbf{H}_{\alpha, X}} \leqq|\log \eta|^{5}\|u\|_{\mathbf{H}_{\alpha, X}} .
$$


An easy extension of this result leads to the bound, for $M \leqq 2 N+2$,

$$
\sup _{J \leqq M}\left\|\partial_{x}^{j} L^{-1} u\right\|_{\mathbf{H}_{\alpha, X}} \leqq|\log \eta|^{5} \sup _{J \leqq M}\left\|\partial_{x}^{j} u\right\|_{\mathbf{H}_{\alpha, X}} .
$$

We next analyze $K$. Let us assume that

$$
\sup _{j \leqq M}\left\|\partial_{x}^{j} u\right\|_{\mathbf{H}_{\alpha, X}} \leqq 1 \quad \text { for } M \leqq 2 N+2 .
$$

Then it is immediate from the definition of $K$ that

$$
\sup _{j \leqq M}\left\|\partial_{x}^{j} K u\right\|_{\mathbf{H}_{\alpha, X}} \leqq \mathbf{O}(1)\left(\eta \sup _{j \leqq M+4}\left\|\partial_{x}^{j} u\right\|_{\mathbf{H}_{\alpha, X}}+\sup _{j \leqq M}\left\|\partial_{x}^{j} u\right\|_{\mathbf{H}_{\alpha, X}}^{2}\right) .
$$

Since $K$ is multilinear, we find by polarization, that

$$
\begin{aligned}
& \sup _{j \leqq M}\left\|\partial_{x}^{j}\left(K(u)-K\left(u^{\prime}\right)\right)\right\|_{\mathbf{H}_{\alpha, X}} \\
& \quad \leqq \mathbf{O}(1) \sup _{j \leqq M+4}\left\|\partial_{x}^{j}\left(u-u^{\prime}\right)\right\|_{\mathbf{H}_{\alpha, X}}\left(\eta+\sup _{j \leqq M}\left(\left\|\partial_{x}^{j} u\right\|_{\mathbf{H}_{\alpha, X}}+\left\|\partial_{x}^{j} u^{\prime}\right\|_{\mathbf{H}_{\alpha, X}}\right)\right) .
\end{aligned}
$$

It is here that we shall gain a small factor per iteration, see below. Note now that

$$
\sup _{j \leqq 2 N+4}\left\|\partial_{x}^{j} v\right\|_{\mathbf{H}_{\alpha, X}} \leqq \eta|\log \eta|^{5} .
$$

Combining (10.3), (10.5) and (10.6), we get recursively for $2 \leqq p \leqq N+1$, the two inequalities

$$
\begin{gathered}
\sup _{j \leqq 2(N+1-p)}\left\|\partial_{x}^{j} u_{p}\right\|_{\mathbf{H}_{\alpha, X}} \leqq \mathbf{O}(1) \eta|\log \eta|^{5}, \\
\sup _{j \leqq 2(N+1-p)+2}\left\|\partial_{x}^{j}\left(u_{p}-u_{p-1}\right)\right\|_{\mathbf{H}_{\alpha, X}} \leqq \mathbf{O}(1) \eta|\log \eta|^{5} \sup _{J \leqq 2(N+2-p)+2}\left\|\partial_{x}^{j}\left(u_{p-1}-u_{p-2}\right)\right\|_{\mathbf{H}_{\alpha, X}} .
\end{gathered}
$$

The assertion of the proposition follows from

$$
L u_{N}-K\left(u_{N}\right)-v=L u_{N}-L u_{N+1} .
$$

\section{The Fixed Point Problem}

Before proceeding with the problem, we summarize the situation as it appears at this point. In Sect. 4, we transformed the existence problem for the front to the form (cf. (4.19)),

$$
B_{\omega} w_{1}-w_{1}\left|w_{1}\right|^{2}=h_{1}(\mathbf{w})
$$

and (cf. (4.15)),

$$
A_{\omega n} w_{n}=h_{n}(\mathbf{w}), \quad n \neq \pm 1 .
$$

In previous sections, we have solved the problems

$$
B_{\omega} w_{1}-w_{1}\left|w_{1}\right|^{2}=h
$$


and

$$
A_{\omega n} w_{n}=h
$$

for any given $h$ (in the appropriate space $\mathbf{H}_{\alpha, X}$ ), by giving explicit inverses, on $(-\infty, X]$. In the case of Eq. (11.4), the inverse has been defined by the integral (7.25). We call this particular inverse $A_{\omega n}^{-1}$. Note, however, that for $n \neq \pm 1, A_{\omega n}$ has a two dimensional null space in $\mathbf{H}_{\alpha, X}$, which is spanned by the functions

$$
e^{i q_{n, 1} x} \text {, and } e^{i q_{n, 2} x}
$$

where $q_{n, 1}$ and $q_{n, 2}$ are the two roots of the characteristic polynomial (7.1) with negative imaginary part. We denote

$$
T_{n, \xi, \zeta}=\xi e^{i q_{n, 1}(x-X)}+\zeta e^{i q_{n, 2}(x-X)} .
$$

The most general solution of (11.4) for fixed $h$ is then

$$
w_{n}=A_{\omega n}^{-1} h-T_{n, \xi, \zeta}, \quad \xi, \zeta \in \mathbb{C} .
$$

(The requirement that $w_{n}=\bar{w}_{-n}$ will force relations between the $\xi$ and $\zeta$ for $n$ and $-n$.)

In the case $n=1$ (we shall not explicitly mention the case of negative $n$ any more), the situation is similar, but somewhat more complex. In Sect.10, we found an approximate solution $(l, 0)+u_{N}$ for Eq. $(9.1)$, with $h=0$. We make the ansatz

$$
w_{1}=\left(\begin{array}{l}
l \\
0
\end{array}\right)+u_{N}+\left(\begin{array}{l}
a \\
b
\end{array}\right) \text {. }
$$

We find for given $h$ the equation

$$
L\left(\begin{array}{l}
a \\
b
\end{array}\right)=\left(\begin{array}{l}
k_{11} \\
k_{21}
\end{array}\right)+\left(\begin{array}{l}
K_{12}+\operatorname{Re} h \\
K_{22}+\operatorname{Im} h
\end{array}\right) .
$$

The terms $K_{j 2}$ are obtained as follows. We set (instead of (9.4))

$$
z=l+u_{1 N}+a+i\left(u_{2 N}+b\right) \text {. }
$$

Substituting into (9.1), we get an equation of the form (9.5) with the same left-hand side but with different right-hand sides. We find

$$
K_{j 2}=K_{j 2 I}+K_{j 2 J}+K_{j 2 K}, \quad \text { for } j=1,2
$$

where

$$
\left(\begin{array}{l}
K_{12 I} \\
K_{22 I}
\end{array}\right)=L u_{N}-K\left(u_{N}\right)-v
$$

and

$$
\begin{aligned}
K_{12 K}= & 6\left(1-\omega^{2}\right) a^{\prime \prime}-4 \frac{1-\omega^{2}}{\eta} \omega a^{\prime} \\
& +\frac{\left(1-\omega^{2}\right)^{2}}{\eta^{2}} a+3 a^{2} l+a^{3}+a b^{2}+b^{2} l
\end{aligned}
$$




$$
\begin{aligned}
K_{22 K}= & 6\left(1-\omega^{2}\right) b^{\prime \prime}-4 \frac{1-\omega^{2}}{\eta} b^{\prime} \\
& +\frac{\left(1-\omega^{2}\right)^{2}}{\eta^{2}} b+2 a b l+a^{2} b-b^{3}
\end{aligned}
$$

Finally, the terms coming from $u_{N}$ occur in $K_{j 2 J}$, which is a bounded operator from a ball of radius 1 in $\mathbf{H}_{\alpha, X} \oplus \mathbf{H}_{\alpha, X}$ to $\mathbf{H}_{\alpha, X}$, with norm bounded by $\mathbf{O}\left(\eta|\log \eta|^{5}\right)$. The nullspace of $L$ is easily seen to consist, in $\mathbf{H}_{\alpha, X}$, of the function $\left(l^{\prime}(x), 0\right)$. In fact, all other possible candidates are seen to have the wrong behavior at $-\infty$. An inverse $L^{-1}$ of $L$ has been defined by (9.14) and (9.17), and we denote below $L^{-1}$ this particular inverse. The most general solution of the problem

$$
L\left(\begin{array}{l}
a \\
b
\end{array}\right)=\left(\begin{array}{l}
q_{1} \\
q_{2}
\end{array}\right)
$$

for given $q_{1}, q_{2}$ is therefore

$$
\left(\begin{array}{l}
a \\
b
\end{array}\right)=L^{-1}\left(\begin{array}{l}
q_{1} \\
q_{2}
\end{array}\right)-\tau\left(\begin{array}{c}
l^{\prime} / l^{\prime}(0) \\
0
\end{array}\right)=L^{-1}\left(\begin{array}{l}
q_{1} \\
q_{2}
\end{array}\right)-T_{\tau},
$$

where we define

$$
T_{\tau}=\tau\left(\begin{array}{c}
l^{\prime} / l^{\prime}(0) \\
0
\end{array}\right)
$$

We next consider the term

$$
-L^{-1}\left(\begin{array}{l}
k_{11} \\
k_{21}
\end{array}\right)=(\mathbf{M}-1)\left(\begin{array}{l}
a \\
b
\end{array}\right)-\left(\begin{array}{l}
\mathbf{O}_{1}(a, b) \\
\mathbf{O}_{2}(a, b)
\end{array}\right),
$$

cf. Eq. (9.44). Here, $\mathbf{O}_{1}, \mathbf{O}_{2}$ were given in (9.45). We denote $\mathbf{M}^{-1}$ the inverse of $\mathbf{M}$ given by (9.50). Then the equation

$$
\mathbf{M}\left(\begin{array}{l}
a \\
b
\end{array}\right)=\left(\begin{array}{l}
t_{1} \\
t_{2}
\end{array}\right)
$$

for given $t_{1}, t_{2}$ has the solution

$$
\left(\begin{array}{l}
a \\
b
\end{array}\right)=\mathbf{M}^{-1}\left(\begin{array}{l}
t_{1} \\
t_{2}
\end{array}\right)-T_{1, \xi, \zeta}
$$

where $T_{1, \xi, \zeta}=\xi v_{1}+\zeta v_{2}$, and $v_{1}, v_{2}$ are the two eigenvectors of $\mathbf{M}$ with eigenvalue zero (corresponding to $p_{2}, p_{4}$ in (9.48)).

In the interval $[X, \infty)$, we viewed the equation of the fronts as a dynamical system. Equations (4.6) can be written as a flow

$$
\frac{d Y}{d t}=A Y+F(Y)
$$

where

$$
Y=\left\{w_{n}^{(m)} \mid n \in \mathbb{Z}, \quad m=0,1,2,3\right\},
$$


and the upper indices denote derivatives. $A$ is the obvious operator induced from the left-hand side of (4.10) while $F$ is the (non-linear) operator induced from its righthand side.

We now formulate the existence problem for the fronts in terms of a fixed point problem, in a space of the functions $w_{n}, n \neq \pm 1$, and $a, b$, augmented by parameters $\xi_{n}, \zeta_{n}, \tau$. The construction will be based on the following trivial but important observation. Assume $O$ is an operator (such as $L, \mathbf{M}$, or $A_{\omega n}$ ), and that $g$ is in its null space, i.e. $O g$. Denote by $O^{-1}$ an inverse of $O$. Then the following is true: For every $\zeta \in \mathbb{C}$, if $f$ solves

$$
f+\zeta g=O^{-1} h
$$

(assuming $O^{-1} h$ is defined), one has

$$
\text { of }-h=0 \text {. }
$$

This is the way in which we shall use the free parameters $\xi, \zeta, \tau$, which we discussed above.

We shall now define two Banach spaces $\mathbf{F}$ and $\mathbf{G}$, and a map $\mathbf{S}$ from a ball $B_{0}$ around 0 in $\mathbf{F}$ to $\mathbf{G}$. The map $\mathbf{S}$ will be seen to be differentiable. Define $r$ by

$$
r=\|\mathbf{S}(0)\|_{\mathbf{G}} \text {. }
$$

We shall show that there is a number $\sigma>0$ such that

1) The ball $B_{\mathbf{F}, \sigma}$ of radius $\sigma$ in $\mathbf{F}$ is contained in $\mathbf{B}_{0}$.

2) $r<\sigma \inf _{y \in B_{\mathbf{F}, \sigma}}\left\|\left(\mathbf{D S}_{y}\right)^{-1}\right\|^{-1}$.

These two conditions imply the existence of a $y_{0} \in B_{\mathbf{F}, \sigma}$ such that $\mathbf{S}\left(y_{0}\right)=0$. The map $\mathbf{S}$ will be constructed in such a way that $y_{0}$ will produce a solution of the system (4.6).

The number $\rho_{1}>0$ will be fixed later on. We shall not mention any more the condition $w_{-n}=\bar{w}_{n}$, and we shall only consider $n \geqq 0, n$ odd.

Definition of $\mathbf{F}$. The space $\mathbf{F}$ is a direct sum of two Banach spaces $\mathbf{F}=\mathbf{F}_{1} \oplus \mathbf{F}_{2}$, where

$$
\begin{array}{ll}
\mathbf{F}_{1}=\left\{\mathbf{w}=\left(a, b,\left(w_{n}\right)_{n \in\{3,5,7, \ldots\}}\right),\right. & \left.a, b, w_{n} \in \mathbf{H}_{\alpha, X}\right\}, \\
\mathbf{F}_{2}=\left\{\boldsymbol{\Xi}=\left(\tau,\left(\xi_{n}, \zeta_{n}\right)_{n \in\{1,3,5, \ldots\}}\right),\right. & \left.\tau \in \mathbb{R}, \xi_{n}, \zeta_{n} \in \mathbb{C}\right\},
\end{array}
$$

with the norms

$$
\begin{aligned}
& \|\mathbf{W}\|_{\mathbf{F}_{1}}=\|a\|_{\mathbf{H}_{\alpha, X}} \eta^{-\rho_{1}}+\|b\|_{\mathbf{H}_{\alpha, X}} \eta^{-\rho_{1}}+\sup _{n \geqq 3} \eta^{-\rho_{1}|n|}|n|^{3}\left\|w_{n}\right\|_{\mathbf{H}_{\alpha, X}}, \\
& \|\boldsymbol{\Xi}\|_{\mathbf{F}_{2}}=m(X)\left\{\sup _{n \geqq 1} \eta^{-\rho_{1} n} n^{3}\left(\left|\xi_{n}\right|+\left|\zeta_{n}\right|\right)\right\}+|\tau| .
\end{aligned}
$$

In the above definition, there is a slight abuse of notation, since we used $\mathbf{w}$ earlier to denote the set of $w_{n}$ for all $n$ including $n= \pm 1$, while we now switch to the translated variables $a$ and $b$. We define the notation $w_{1}=l+a+i b$, where $l$ denotes henceforth what used to be called $l+u_{N}$. The factor $m(X)$ serves balancing purposes. 
Definition of $\mathbf{G}$. The space $\mathbf{G}$ is a direct sum of three Banach spaces $\mathbf{G}=$ $\mathbf{G}_{1} \oplus \mathbf{G}_{2} \oplus \mathbf{G}_{3}$. The first will break translation invariance, the second will be isomorphic to $\mathbf{F}_{1}$, and the third will be identified with the derivatives of the $w_{n}$ at $X$, so that the theory of Sect. 8 can be naturally applied. We consider therefore vectors $\mathbf{X}$ of the form

$$
\mathbf{X}=\left(x_{n}\right)_{n \in\{1,3,5, \ldots\}}, \quad x_{n} \in \mathbb{R}^{4},
$$

and we define a norm on such vectors:

$$
\|\mathbf{X}\|_{\mathbf{G}_{3}}=m(X) \sup _{n} \eta^{-\rho_{1} n} \sup _{0 \leqq m \leqq 3} n^{3}\left|x_{n, m}\right| \gamma_{n}^{-m},
$$

where $\gamma_{n}=|\mu| / \eta$ (an approximation to the root of maximum modulus of (7.1)), $\mu \equiv \omega n$.

Definition. There is a natural map $\mathbf{I}$ from $\mathbf{X}$ to $\mathbf{B}$, as defined in Sect. 8. The norm of $\mathbf{I}$ is $1 / m(X)$ and the norm of $\mathbf{I}^{-1}$ is $m(\mathbf{X})$. We consider in $\mathbf{B}$ the space $E^{u}$, i.e. the unstable subspace of the family of operators induced by the $\left\{A_{\omega n}\right\}$ on these derivatives. This space is a direct sum of two dimensional subspaces for each of the components of $\mathbf{B}$, except for $n=1$, where it is 1-dimensional. We denote by $P^{u}$ the corresponding projection. We now define $P_{0}^{u}$ to be the projection of $\mathbf{I}^{-1} P^{u} \mathbf{B}$ onto the vectors whose component $x_{1,0}$ equals 0 . The component $x_{1,0}$ of $\mathbf{X}$ will be considered as an element in the Banach space $\mathbf{G}_{1}$. We now define

$$
\begin{aligned}
& \mathbf{G}_{1}=\mathbb{R}, \\
& \mathbf{G}_{2}=\mathbf{F}_{1}, \\
& \mathbf{G}_{3}=P_{0}^{u} \mathbf{I}^{-1} P^{u} \mathbf{B} .
\end{aligned}
$$

Definition of $\mathbf{S}$. The operator $\mathbf{S}$ has three components, corresponding to the components of $\mathbf{G}$. The first component is

$$
\mathbf{S}^{(1)}(\mathbf{w}, \Xi)=S_{1} l(0)+S_{1}(a(0)+i b(0))-S_{1} / 2 \text {. }
$$

If $\mathbf{S}^{(1)}(\mathbf{w}, \Xi)=0$, then we have broken the translation invariance of the solution, by fixing $W_{1}(0)=S_{1} / 2$. We define the second component of the operator $\mathbf{S}$ by giving its image on each of the components of $\mathbf{F}_{1}$. For $n \neq 1$, we define

$$
\mathbf{S}_{n}^{(2)}(\mathbf{w}, \Xi)=w_{n}-A_{\omega n}^{-1} h_{n}(\mathbf{w})+T_{n, \xi_{n}, \zeta_{n}},
$$

and for $n=1$ we define

$$
\begin{gathered}
\mathbf{S}_{1}^{(2)}(\mathbf{w}, \Xi)=\left(\begin{array}{l}
a \\
b
\end{array}\right)+\mathbf{M}^{-1} T_{\tau}+T_{1, \xi_{1}, \zeta_{1}} \\
-\mathbf{M}^{-1}\left(L^{-1}\left(\begin{array}{l}
\operatorname{Re} h_{1}(\mathbf{w})+K_{12}(\mathbf{w}) \\
\operatorname{Im} h_{1}(\mathbf{w})+K_{22}(\mathbf{w})
\end{array}\right)-\left(\begin{array}{l}
\mathbf{O}_{1}(\mathbf{w}) \\
\mathbf{O}_{2}(\mathbf{w})
\end{array}\right)\right) .
\end{gathered}
$$

Before proceeding with the definitions, we verify that $\mathbf{S}_{1}^{(2)}(\mathbf{w}, \Xi)=0$ implies that we have solved (9.1) (on $(-\infty, X]$ ). Indeed, using (11.14), and (11.11), we see upon multiplying by $\mathbf{M}$ that

$$
\mathbf{M}\left(\begin{array}{l}
a \\
b
\end{array}\right)+T_{\tau}=L^{-1}\left(\begin{array}{l}
\operatorname{Re} h_{1}(\mathbf{w})+K_{12}(\mathbf{w}) \\
\operatorname{Im} h_{1}(\mathbf{w})+K_{22}(\mathbf{w})
\end{array}\right)-\left(\begin{array}{l}
\mathbf{O}_{1}(\mathbf{w}) \\
\mathbf{O}_{2}(\mathbf{w})
\end{array}\right) .
$$


Using now (11.10), we see that

$$
\left(\begin{array}{l}
a \\
b
\end{array}\right)+T_{\tau}=L^{-1}\left(\begin{array}{l}
k_{11}+K_{12} \\
k_{21}+K_{22}
\end{array}\right)+L^{-1} h_{1}(\mathbf{w}) .
$$

Applying again (11.14), and (11.6)-(11.8), we see that we have solved (9.1).

Finally, we define $\mathbf{S}^{(3)}$ by

$$
\mathbf{S}^{(3)}(\mathbf{w}, \Xi)=P_{0}^{u}\left(\mathbf{I}^{-1} P^{u} \mathbf{I T}(\mathbf{w}, \Xi)-\mathbf{I}^{-1} \Phi\left(\left(1-\mathbf{P}^{u}\right) \mathbf{I T}(\mathbf{w}, \Xi)\right)\right) .
$$

Here, we define for $n \geqq 3,0 \leqq m \leqq 3$

$$
\mathbf{T}_{n}^{(m)}(\mathbf{w}, \Xi)=\left(-i \partial_{x}\right)^{m}\left(-\mathbf{S}_{n}^{(2)}(\mathbf{w}, \Xi)+w_{n}+S_{n} l\right)(\mathbf{X}),
$$

while for $n=1$, we define

$$
\mathbf{T}_{1}^{(m)}(\mathbf{w}, \Xi)=S_{1}\left(-i \partial_{x}\right)^{m}\left(-\left(\mathbf{1}-\mathbf{M}^{-1} \mathbf{Q}\right) \mathbf{S}_{1}^{(2)}(\mathbf{w}, \Xi)+\left(\begin{array}{l}
a \\
b
\end{array}\right)+\left(\begin{array}{l}
l \\
0
\end{array}\right)\right)(\mathbf{X}) .
$$

Here $\mathbf{Q}$ is defined as follows: In Sect. 9, we have found in Eq. (9.45) terms $\eta^{2} \mathbf{O}_{i 2}$. These are linear operators acting on the pair $(a, b)$. The operator $\mathbf{Q}$ is defined by

$$
\mathbf{Q}\left(\begin{array}{l}
u \\
v
\end{array}\right)=\eta^{2}\left(\begin{array}{l}
\mathbf{O}_{12}(u, v) \\
\mathbf{O}_{22}(u, v)
\end{array}\right) \text {. }
$$

The existence of the function $\Phi$ has been shown in Sect. 8. The above definitions (11.26), (11.27) are somewhat more complicated than might seem necessary, but they will make the estimates less difficult. In particular, the terms linear in $w$ are cancelled, and by the results of Sect. 9 this implies that the derivatives in (11.26), (11.27) exist. We again note that if $\mathbf{S}^{(3)}(\mathbf{w}, \Xi)=0$, then we will have solved the matching problem at $X$. The constant $X$ was fixed to be $C \log \left(\eta^{-1}\right)$ in Eq. (9.8).

\section{Bounds on the Approximate Solution}

Here, we bound $\mathbf{S}(0)$, but we simultaneously estimate some of the terms of $\mathbf{S}(\mathbf{w}, \Xi)$ when

$$
\|\mathbf{w}\|_{\mathbf{F}_{1}}+\|\Xi\|_{\mathbf{F}_{2}} \leqq \sigma
$$

for some (small) $\sigma$. We start by bounding the inhomogeneities $h_{n}$. By the results of Sect. 3, we have, for $n \neq \pm 1$,

$$
\left|S_{n}\right|=\left|s_{n}\right| \leqq \eta^{2 \rho(|n|+1)}
$$

and, when $n=1$,

$$
\left|s_{1}\right|<\eta^{8 \rho}
$$

where $0<\rho<1 / 4$ can be chosen arbitrarily. We shall choose $\rho=1 / 5$ henceforth. We have, for $n \neq \pm 1$, by (4.13), (4.14),

$$
W_{n}(x)=S_{n} l(x)+w_{n}(x)
$$


so that, by (12.2),

$$
\sup _{x \in(-\infty, X]}\left|W_{n}(x)\right| \leqq \mathbf{O}\left(\eta^{2 \rho(|n|+1)}+\sigma \eta^{\rho_{1}|n|}\right)
$$

and

$$
\left\|W_{n}-S_{n} l\right\|_{\mathbf{H}_{\alpha, X}} \leqq \mathbf{O}\left(\sigma \eta^{\rho_{1}|n|} /|n|^{3}\right) .
$$

By (4.11) and (3.7), we find, with $\Gamma$ defined by (3.3),

$$
W_{1}=\left(\Gamma+s_{1}\right)(l+a+i b)=S_{1}(l+a+i b),
$$

so that

$$
\sup _{x \in(-\infty, X]}\left|W_{1}(x)\right| \leqq \mathbf{O}(1)
$$

and

$$
\left\|W_{1}-S_{1} l\right\|_{\mathbf{H}_{\alpha, X}} \leqq \mathbf{O}\left(\sigma \eta^{\rho_{1}}\right) .
$$

The expansion of $h_{n}(\mathbf{w})$ leads to the following expression (all sums are over $p+q+r=n)$

$$
\begin{aligned}
h_{n}(\mathbf{w})= & \eta^{2} \sum_{p, q, r \neq \pm 1}\left(S_{p} l+w_{p}\right)\left(S_{q} l+w_{q}\right)\left(S_{r} l+w_{r}\right) \\
& +3 \eta^{2} \sum_{\substack{p, q \neq \pm 1 \\
r= \pm 1}}\left(S_{p} l+w_{p}\right)\left(S_{q} l+w_{q}\right) S_{1}(l+a+r i b) \\
& +3 \eta^{2} \sum_{\substack{p, p= \pm 1 \\
q, r= \pm 1}}\left(S_{p} l+w_{p}\right) S_{1}^{2}(l+a+q i b)(l+a+r i b) \\
& +\eta^{2} \sum_{p, q, r= \pm 1} S_{1}^{3}(l+a+p i b)(l+a+q i b)(l+a+r i b)-S_{n} A_{\omega n} l .
\end{aligned}
$$

(The last sum is only present if $n= \pm 3$.) Note now, that by the construction of the stationary solution,

$$
\left(\eta^{2}-\left(1+n^{2} \omega^{2}\right)^{2}\right) S_{n}=\eta^{2} \sum_{p+q+r=n} S_{p} S_{q} S_{r}
$$

When $\mathbf{w}=0$, we have, by (12.11),

$$
h_{n}(0)=\eta^{2} \sum_{p+q+r=n} S_{p} S_{q} S_{r} l^{3}-S_{n} A_{\omega n} l=S_{n}\left(\left(\eta^{2}-\left(1+\eta^{2} \omega^{2}\right)^{2}\right) l^{3}-A_{\omega n} l\right) .
$$

The terms in (12.10) which do depend on w are bounded as follows, using (12.5), (12.6), and the fact that $\sigma$ is small

$$
\begin{aligned}
|n|^{3}\left\|h_{n}(\mathbf{w})-h_{n}(0)\right\|_{\mathbf{H}_{\alpha, X}} & =\mathbf{O}\left(\sigma \eta^{2}\right)\left\{\eta^{\rho_{1}|n|}+\eta^{\rho_{1}(|n|-1)}+\eta^{\rho_{1}(|n|-2)}+\delta_{3, n} \eta^{\rho_{1}}\right\} \\
& \leqq \mathbf{O}\left(\sigma \eta^{2-2 \rho_{1}+\rho_{1}|n|}\right) .
\end{aligned}
$$

Note that the sum over $p+q+r=n$ in the definition of $h_{n}$ has been absorbed by the 
bounds (12.2), (12.3), (12.6), since

uniformly in $\eta$.

$$
\sum_{\substack{p+r=n \\ p, q, r \neq 0}} \frac{\eta^{\rho_{1}(|p|+|q|+|r|-|n|)}}{p^{3} q^{3} r^{3}} \leqq \mathbf{O}\left(n^{-3}\right),
$$

We next analyze $h_{1}(\mathbf{w})$. We have, cf. (4.19),

$$
h_{1}(\mathbf{w})=\frac{1}{S_{1}}\left(\sum^{*} W_{p} W_{q} W_{r}-w_{1}\left|w_{1}\right|^{2}\left(1-3 S_{1}^{2}\right)\right),
$$

where $w_{1}=l+a+i b$. Recall that $\sum^{*}$ is the sum over $|p|+|q|+|r| \neq 3$. As in the case of $h_{n}, n \neq \pm 1$, we can expand

$$
\begin{aligned}
h_{1}(\mathbf{w})= & \sum_{p, q, r \neq \pm 1}^{*}\left(S_{p} l+w_{p}\right)\left(S_{q} l+w_{q}\right)\left(S_{r} l+w_{r}\right) \\
& +3 \sum_{\substack{p, q \neq \pm 1 \\
r= \pm 1}}^{*}\left(S_{p} l+w_{p}\right)\left(S_{q} l+w_{q}\right) S_{1}(l+a+r i b) \\
& +3 \sum_{\substack{p \neq \pm \pm 1 \\
q, r= \pm 1}}^{*}\left(S_{p} l+w_{p}\right) S_{1}^{2}(l+a+q i b)(l+a+r i b)-w_{1}\left|w_{1}\right|^{2}\left(1-3 S_{1}^{2}\right) .
\end{aligned}
$$

Similarly, when $\mathbf{w}=0$, using (12.2), (12.3), which imply, among other things, $1-3 S_{1}^{2}=\mathbf{O}\left(\eta^{8 \rho}\right)$, we get

$$
h_{1}(0)=\sum^{*} S_{p} S_{q} S_{r} l^{3}-l^{3}\left(1-3 S_{1}^{2}\right)=\mathbf{O}\left(\eta^{2 \sigma(3+1)}+\eta^{8 \rho}\right) l^{3}=\mathbf{O}\left(\eta^{8 \rho}\right) l^{3} .
$$

Also,

$$
\left\|h_{1}(\mathbf{w})-h_{1}(0)\right\|_{\mathbf{H}_{\alpha, X}}=\mathbf{O}\left(\sigma \eta^{2 \rho(3+1)}\right)+\mathbf{O}\left(\sigma \eta^{8 \rho}\right)=\mathbf{O}\left(\sigma \eta^{8 \rho}\right)
$$

We now start bounding $\mathbf{S}(0)$ itself. This means in particular that in the above estimates $\mathbf{w}=0$. We first consider $\mathbf{S}^{(1)}$. Note that $l(0)=1 / 2$. Therefore, using the definition (11.22), we find

$$
\mathbf{S}^{(1)}(0,0)=0 \text {. }
$$

Next, we consider $\mathbf{S}_{n}^{(2)}$, when $n \neq \pm 1$. We have, using the identities leading to Eq. (3.8),

$$
\begin{aligned}
\mathbf{S}_{n}^{(2)}(0,0) & =-A_{\omega n}^{-1}\left(\eta^{2} \sum_{p+q+r=n} l^{3} S_{p} S_{q} S_{r}-S_{n} A_{\omega n} l\right) \\
& =-A_{\omega n}^{-1}\left(l^{3}\left(\eta^{2}-\left(1-n^{2} \omega^{2}\right)^{2}\right) S_{n}-S_{n} A_{\omega n} l\right) \equiv-S_{n} A_{\omega n}^{-1} l_{n},
\end{aligned}
$$

where $l_{n}$ is a polynomial in the derivatives of $l$ (up to fourth order) with coefficients which are bounded by $\mathbf{O}\left(n^{4}\right)$. We now note that

$$
\left\|\partial^{j} l_{n}\right\|_{\mathbf{H}_{\alpha, X}}=\mathbf{O}\left(n^{4}\right), \text { for } j=0, \ldots, 4 \text {. }
$$

Therefore, we have, by Lemma 7.4,

$$
\left\|A_{\omega n}^{-1} l_{n}\right\|_{\mathbf{H}_{\alpha, X}} \leqq \mathbf{O}\left(n^{4} \eta^{-1 / 2}\right) .
$$

We finally analyze $\mathbf{S}_{1}^{(2)}(0,0)$. Since $\mathbf{O}_{1}$ and $\mathbf{O}_{2}$ are linear in $a$ and $b$, we have

$$
\mathbf{S}_{1}^{(2)}(0,0)=-\mathbf{M}^{-1} L^{-1}\left(\begin{array}{l}
\operatorname{Re} h_{1}(0)+K_{12}(0) \\
\operatorname{Im} h_{1}(0)+K_{22}(0)
\end{array}\right) \text {. }
$$


We have bounded $h_{1}$ in (12.17), and we consider now $K_{12}$ and $K_{22}$. Note that $K_{12}(0)$ and $K_{22}(0)$ are in $\mathbf{H}_{\alpha, X}$. For later use, we also note that $L_{j}^{-1} K_{j 2}(\mathbf{w})$ is of the form

$$
L_{j}^{-1} K_{j 2}(\mathbf{w})=\eta\left(\partial_{x}-g\right)^{-1} \hat{\mathbf{O}}_{j 1}+\eta^{2} \hat{\mathbf{O}}_{j 2}+L_{j}^{-1} \hat{\mathbf{O}}_{j 3},
$$

where the $\hat{\mathbf{O}}_{i j}, j=1,2$, are bounded linear operators from $\mathbf{H}_{\alpha, X} \oplus \mathbf{H}_{\alpha, X}$ to $\mathbf{H}_{\alpha, X}$, with norm bounded by $\mathbf{O}\left(|\log \eta|^{5}\right)$, and $g=l^{\prime \prime} / l^{\prime}$ or $g=l^{\prime} / l$ and $\hat{\mathbf{O}}_{j 3}$ has norm $\mathbf{O}\left(\|\mathbf{w}\|_{\mathbf{F}_{1}}\right)$ when $\|\mathbf{w}\|_{\mathbf{F}_{1}} \leqq 1$. This follows by obvious (easier) variants of the calculations leading to Proposition 9.3 and to Eq. (9.45). Applying now Proposition 9.1, 9.4 and 10.1 to the terms $h_{1}$ and $K_{j 2}$, we see that

$$
\eta^{-\rho_{1}}\left\|\mathbf{S}_{1}^{(2)}(0,0)\right\|_{\mathbf{H}_{\alpha, X}} \leqq \mathbf{O}\left(\eta^{8 \rho-\rho_{1}-1 / 2}+\eta^{N-1-\rho_{1}-1 / 2}\right)|\log \eta|^{10} \leqq \mathbf{O}\left(\eta^{1 / 2+\rho_{1}}\right),
$$

provided we take $8 \rho-1>2 \rho_{1}$ and $N$ of Sect. 10 sufficiently large. We can now take the sup over $n$ and we find

$$
\begin{aligned}
\eta^{-\rho_{1}} & \left\|\mathbf{S}_{1}^{(2)}(0,0)\right\|_{\mathbf{H}_{\alpha, X}}+\sup _{n \geqq 3} n^{3} \eta^{-\rho_{1} n}\left\|\mathbf{S}_{n}^{(2)}(0,0)\right\|_{\mathbf{H}_{\alpha, X}} \\
& \leqq \mathbf{O}\left(\eta^{1 / 2+\rho_{1}}\right)+\mathbf{O}(1) \sup _{n \geqq 3} \eta^{-\rho_{1} n} n^{7}\left|S_{n}\right| \eta^{-1 / 2} \\
& \leqq \mathbf{O}\left(\eta^{1 / 2+\rho_{1}}\right)+\mathbf{O}\left(\eta^{\rho}\right) \sup _{n \geqq 3} \eta^{\left(\rho-\rho_{1}\right) n} n^{7} \eta^{-\rho(n+1)}\left|S_{n}\right| \eta^{-1 / 2+\rho(n+1)} \\
& \leqq \mathbf{O}\left(\eta^{1 / 2+\rho_{1}}\right),
\end{aligned}
$$

provided we choose $4 \rho_{1}<3 \rho-1 / 2$. Summarizing, we have finally found

$$
\left\|\mathbf{S}^{(2)}(0,0)\right\|_{\mathbf{F}_{1}}=\mathbf{O}\left(\eta^{1 / 2+\rho_{1}}\right) \text {. }
$$

We study next the third component of $\mathbf{S}$. We begin by analyzing the case $n \neq \pm 1$, and we fix $n$. Recall that $P^{u}$ and $P^{s}$ are the spectral projections of $A_{\omega n}$ onto the unstable and stable subspaces, cf. Sect. 7. We have bounded these projections in (7.45). We can now consider the term $S_{n} l$ in $\mathbf{T}_{n}$, cf. (11.26). Then we have

$$
\begin{aligned}
& \left\|P_{n}^{(j)}\left\{\left(-i \partial_{x}\right)^{m} S_{n} l\right\}_{m=0, \ldots, 3}\right\|_{\mathbf{G}_{3}} \\
& \quad \leqq \mathbf{O}(1) m(X) \sup _{0 \leqq m \leqq 3}\left|P_{n, m}^{(j)} \|\left(\left(-i \partial_{x}\right)^{m} l\right)(X)\right|\left|S_{n}\right| \eta^{-\rho_{1}|n|} \sup _{0 \leqq m^{\prime} \leqq 3}\left|\frac{\eta}{\mu}\right|^{m^{\prime}}\left|q_{j}^{m^{\prime}}\right| n^{3}
\end{aligned}
$$

Using (12.2) and the bounds (7.47), (7.48), this is bounded by

$$
\begin{aligned}
& \mathbf{O}(1) m(X) \eta^{-\rho_{1}|n|+2 \rho(|n|+1)} \eta^{4}\left(\frac{\mu}{\eta}\right)^{3} \eta^{-3 / 2} \mu^{-1 / 2} \eta^{3} X e^{-X / 2} \\
& \quad \leqq \mathbf{O}\left(\eta^{2 \rho}\right) \eta^{\left(2 \rho-\rho_{1}\right)(|n|-3)} \eta^{6 \rho-3 \rho_{1}-1 / 2} n^{3} \leqq \mathbf{O}\left(\eta^{2 \rho}\right),
\end{aligned}
$$

provided $2 \rho-1 / 6>\rho_{1}$.

We next consider the other term in $\mathbf{T}_{n}$, namely $\mathbf{S}_{n}^{(2)}(0,0)=-S_{n} A_{\omega n}^{-1} l_{n}$, cf. $(11.26)$ and (12.20). We have

$$
\begin{aligned}
\left\|P_{n}^{(j)} S_{n} A_{\omega n}^{-1} l_{n}\right\|_{\mathbf{G}_{3}} \leqq & \mathbf{O}(1) m(X) \sup _{0 \leqq m \leqq 3} \mid P_{n, m}^{(j)}\left(-i \partial_{x}\right)^{m} \\
& \times\left(A_{\omega n}^{-1} l_{n}\right)(X)|| S_{n} \mid \eta^{-\rho_{1}|n|} n^{3} \gamma_{n}^{-m} .
\end{aligned}
$$

It is easy to check from the definition of $A_{\omega n}^{-1}$, and using (7.23), that $\partial_{x}$ and $A_{\omega n}^{-1}$ 
commute up to boundary terms at $X$. Leaving the detailed discussion of the latter to the reader, we bound (12.27) by

$$
\mathbf{O}(1) m(X) \eta^{4}\left(\frac{\mu}{\eta}\right)^{3} \eta^{-3 / 2} \mu^{-1 / 2} \eta^{-1 / 2} \mu^{4} \eta^{2 \rho(|n|+1)} \eta^{-\rho_{1}|n|} \mu^{3} X e^{-X / 2}
$$

Using, from left to right, (7.47), (7.48), Lemma 7.4, the definition (12.20) of $l_{n}$, and the bound (12.2) on $S_{n}$. Simple arithmetic leads to the bound

$$
\mathbf{O}\left(\eta^{2 \rho}\right) \mu^{19 / 2} \eta^{-1+6 \rho-3 \rho_{1}} \eta^{\left(2 \rho-\rho_{1}\right)(|n|-3)} .
$$

Thus, if we require $2 \rho-1 / 3>\rho_{1}$, then we have the bound

$$
\left\|P_{n}^{(j)} S_{n} A_{\omega n}^{-1} l_{n}\right\|_{\mathbf{G}_{3}} \leqq \mathbf{O}\left(\eta^{2 \rho}\right) n^{19 / 2} \eta^{\left(2 \rho-\rho_{1}\right)(|n|-3)} .
$$

We next consider the case $n= \pm 1$. Note that by Eq. (11.27), we have

$$
\mathbf{T}_{1}^{(m)}(0,0)=S_{1}\left(-i \partial_{x}\right)^{m}\left(-\left(1-\mathbf{M}^{-1} \mathbf{Q}\right) \mathbf{S}_{1}^{(2)}(0,0)+\left(\begin{array}{l}
l \\
0
\end{array}\right)\right)(\mathbf{X}) .
$$

We begin by bounding the contribution of the second term to $\mathbf{S}^{(3)}$.

$$
\begin{aligned}
P_{1}^{(4)}\left|l, l^{\prime}, l^{\prime \prime}, l^{\prime \prime \prime}\right\rangle & =\left|e_{1,4}\right\rangle \sum_{m=0}^{3} P_{1, m}^{(4)} i^{-m} l^{(m)}(X) \\
& =\frac{\left(-i \partial_{x}-q_{1,1}\right)\left(-i \partial_{x}-q_{1,2}\right)\left(-i \partial_{x}-q_{1,3}\right)}{\left(q_{1,4}-q_{1,1}\right)\left(q_{1,4}-q_{1,2}\right)\left(q_{1,4}-q_{1,3}\right)} l(X)\left|e_{1,4}\right\rangle .
\end{aligned}
$$

Using the bounds (7.35), (7.38), and the decay of $l$, we see that

$$
\| P_{1}^{(4)}\left|l, l^{\prime}, l^{\prime \prime}, l^{\prime \prime \prime}\right\rangle \|_{\mathbf{G}_{3}} \leqq \mathbf{O}\left(\eta^{3 / 2-\rho_{1}}\right) \text {. }
$$

We next consider the term in (11.27) which contains $\mathbf{S}_{1}^{(2)}(0,0)$. Note that its ingredients, $h_{1}(0), K_{j 2}$ are all either derivatives of $l$, or multiples of $l^{3}$, see (12.17), (12.15) and the description of $K_{j 2}$ after (12.22). Hence, using the same arguments as above, we get again a bound of order $\mathbf{O}\left(\eta^{1 / 2}\right)$. Therefore, we see that

$$
\left\|P_{1}^{(4)} \mathbf{T}_{1}(0,0)\right\|_{\mathbf{G}_{3}} \leqq \mathbf{O}\left(\eta^{1 / 2-\rho_{1}}\right) .
$$

We can now combine these bounds with those of (12.30) and take the sup over $n$. We obtain

$$
\left\|P^{u} \mathbf{T}(0,0)\right\|_{\mathbf{G}_{3}} \leqq \mathbf{O}\left(\eta^{3 / 2-\rho_{1}}\right) .
$$

We will have to know that the solution of the problem in $[-\infty, X)$ arrives in this ball when considered as a function in $\mathbf{G}_{3}$, as above. But this will follow from the size of $m(X)$, provided $X>C|\log \eta|$ for some sufficiently large $C$. Indeed, it is easy to see that

$$
\|\mathbf{T}(0,0)\|_{\mathbf{G}_{3}} \leqq \mathbf{O}\left(\eta^{-\rho_{1}}\right) .
$$

By the definition of I this yields

$$
\left\|\left(1-P^{u}\right) \mathbf{I T}(0,0)\right\|_{\mathbf{B}} \leqq \frac{1}{m(X)} \mathbf{O}\left(\eta^{-\rho_{1}}\right) .
$$


We now apply Proposition 8.2, and Theorem 8.1. We get

$$
\left\|\Phi\left(\left(1-P^{u}\right) \mathbf{I T}(0,0)\right)\right\|_{\mathbf{B}} \leqq \mathbf{O}\left(\eta^{-4}\right) \frac{1}{m(X)^{2}} .
$$

It follows that

$$
\left\|\mathbf{S}^{(3)}(0,0)\right\|_{\mathbf{G}_{3}} \leqq \mathbf{O}\left(\eta^{-K}\right) \frac{1}{m(X)}+\mathbf{O}\left(\eta^{3 / 2-\rho_{1}}\right),
$$

where $K>0$ is independent of $\eta$. At this point, we use that $X$ is large, and we get

$$
\left\|\mathbf{S}^{(3)}(0,0)\right\|_{\mathbf{G}_{3}} \leqq \mathbf{O}\left(\eta^{2 \rho-\rho_{1}}\right) \text {. }
$$

If we combine now (12.19), (12.23), and (12.36), we see that we have shown the

Theorem 12.1. There is a $\rho>0$ such that the map $\mathbf{S}$, acting on $(0,0) \in \mathbf{F}_{1} \oplus \mathbf{F}_{2}$ is defined and has an image which is bounded in norm by $\mathbf{O}\left(\eta^{\rho}\right)$, provided $\eta$ is sufficiently small.

Remark. $\rho=1 / 5$ works.

\section{Bounds on the Tangent Map}

In this section, we shall bound the tangent map $\delta \mathbf{S}$. We shall use throughout the following notation. We denote the "variation" of a function $F$ by $\delta F$. This is the Fréchet derivative of $F$. It is a linear operator on the tangent vectors, which we always denote by $(\delta \mathbf{w}, \delta \Xi)$ (and similar notations for their components). We will bound the operator $\delta \mathbf{S}$ on a ball

$$
\|\mathbf{w}\|_{\mathbf{F}_{1}}+\|\Xi\|_{\mathbf{F}_{2}} \leqq \sigma,
$$

and we shall in fact do this for $\sigma=\eta^{1 / 2+\rho_{1 / 2}}$.

Theorem 13.1. For every (w, $\Xi$ ) satisfying (13.1) with $\sigma$ as above, one has

$$
\delta \mathbf{S}=\text { an upper triangular matrix }+ \text { small remainder, }
$$

and this matrix will be given in detail in Eq. (13.16).

Proof. We first bound $\delta h_{n}(\mathbf{w}) \delta \mathbf{w}$. Note that by (12.10), it is easy to elaborate all the terms of this expression, and we get a bound very similar to (12.13), because $h_{n}$ is a polynomial in its arguments, namely

$$
|n|^{3}\left\|\delta h_{n}(\mathbf{w}) \delta \mathbf{w}\right\|_{\mathbf{H}_{\alpha, X}} \leqq \mathbf{O}(1)\|\delta \mathbf{w}\|_{\mathbf{F}_{1}} \eta^{2-2 \rho_{1}+\rho_{1}|n|} .
$$

Next, we consider $\delta h_{1}(\mathbf{w})$. The same remarks as above lead to the bound for $\delta h_{1}$, cf. (12.15), similar to (12.18),

$$
\left\|\delta h_{1}(\mathbf{w}) \delta \mathbf{w}\right\|_{\mathbf{H}_{\alpha, X}} \leqq \mathbf{O}(1)\|\delta \mathbf{w}\|_{\mathbf{F}_{1}} \eta^{8 \rho} .
$$

If we consider (11.24), then we see that $\delta \mathbf{S}_{1}^{(2)}$ has terms of the form

$$
\mathbf{M}^{-1} L^{-1}\left(\begin{array}{c}
\operatorname{Re} \delta h_{1}(\mathbf{w})+\delta K_{12}(\mathbf{w}) \\
\operatorname{Im} \delta h_{1}(\mathbf{w})+\delta K_{22}(\mathbf{w})
\end{array}\right) \text { and } \mathbf{M}^{-1}\left(\begin{array}{c}
\delta \mathbf{O}_{1}(\mathbf{w}) \\
\delta \mathbf{O}_{2}(\mathbf{w})
\end{array}\right) .
$$

In (12.17), (12.18) we have bounded $h_{1}$; in (12.22) we have bounded $L_{j}^{-1} K_{j 2}$; and in 
(9.45) we have bounded $\mathbf{O}_{j}$. All these bounds were without the factor $\mathbf{M}^{-1}$. To bound the variations, we observe that $K_{j 2}$ is linear plus cubic. This implies by polarization a bound on $L_{j}^{-1} \delta K_{j 2}(\mathbf{w}) \delta \mathbf{w}$. Similarly, the $\mathbf{O}_{j}$ are linear, so that bounds follow from (9.45). The action of $\mathbf{M}^{-1}$ is now controlled by applying Proposition 9.4. We finally get the bound

$$
\begin{aligned}
& \eta^{-\rho_{1}}\left\|\delta \mathbf{S}_{1}^{(2)}(\mathbf{w}, \Xi)(\delta \mathbf{w}, \delta \Xi)-\delta\left(\begin{array}{l}
a \\
b
\end{array}\right)-\mathbf{M}^{-1} T_{\delta \tau}-T_{1, \delta \xi_{1}, \delta \zeta_{1}}\right\|_{\mathbf{H}_{\alpha, X}} \\
& \leqq \mathbf{O}(1)\left(\eta^{1 / 2}|\log \eta|^{5}+\mathbf{O}\left(\eta^{-1 / 2} \sigma\right)\right)\left\|\delta \mathbf{w}_{1}\right\|_{\mathbf{F}_{1}} .
\end{aligned}
$$

Note that by the choice of $\sigma$, this is bounded by

$$
\mathbf{O}\left(\eta^{\rho_{1} / 2}\right)\left\|\delta \mathbf{w}_{1}\right\|_{\mathbf{F}_{1}} .
$$

We next consider (13.3). By (7.27), the inverse of $A_{\omega n}$ is bounded by $\mathbf{O}\left(\eta^{-1}\right)$. Therefore, we see that

$$
|n|^{3} \eta^{-\rho_{1}|n|}\left\|A_{\omega n}^{-1} \delta h_{n}(\mathbf{w}) \delta \mathbf{w}\right\|_{\mathbf{H}_{\alpha, X}} \leqq \mathbf{O}(1)\|\delta \mathbf{w}\|_{\mathbf{F}_{1}} \eta^{-1} \eta^{2-2 \rho_{1}}
$$

In terms of $\mathbf{S}_{n}^{(2)}$, this implies

$$
|n|^{3} \eta^{-\rho_{1}|n|}\left\|\delta \mathbf{S}_{n}^{(2)}(\mathbf{w}, \Xi)(\delta \mathbf{w}, \delta \Xi)-\delta w_{n}-T_{n, \delta \xi_{n}, \delta \zeta_{n}}\right\|_{\mathbf{H}_{\alpha, X}} \leqq \mathbf{O}(1)\|\delta \mathbf{w}\|_{\mathbf{F}_{1}} \eta^{1-2 \rho_{1}} .
$$

We next analyze the variation of $\mathbf{S}^{(3)}$. We claim that the only terms with "large" variations in (11.25) are those coming from the operators $\mathbf{T}_{n}$. We begin with the case $n \geqq 3$. We first observe that

$$
\begin{aligned}
\delta \mathbf{T}_{n}^{(m)}(\mathbf{w}, \Xi)(\delta \mathbf{w}, \delta \Xi) & =\left(-i \partial_{x}\right)^{m}\left(-\delta \mathbf{S}_{n}^{(2)}(\mathbf{w}, \Xi)(\delta \mathbf{w}, \delta \Xi)+\delta w_{n}+T_{n, \delta \xi_{n}, \delta \zeta_{n}}\right)(X) \\
& =\left(-i \partial_{x}\right)^{m}\left(A_{\omega n}^{-1} \delta h_{n}(\mathbf{w}) \delta \mathbf{w}+T_{n, \delta \xi_{n}, \delta \zeta_{n}}\right)(X) .
\end{aligned}
$$

By Lemma 7.5, these expressions exist. To bound them, we observe first that

$$
\left(\delta \mathbf{T}_{n}^{(m)}(\mathbf{w}, \Xi)(\delta \mathbf{w}, \delta \Xi)-\left(-i \partial_{x}\right)^{m} T_{n, \delta \xi_{n}, \delta \zeta_{n}}\right)(X)=\left(-i \partial_{x}\right)^{m} A_{\omega n}^{-1} \delta h_{n}(\mathbf{w}) \delta \mathbf{w} .
$$

We consider now the projections of this quantity on $E^{u}$ and $E^{s}$. Using the integral representation Eq. (7.55), we get

$$
\begin{aligned}
& \left\|P_{n, m}^{(j)}\left(\delta \mathbf{T}_{n}^{(m)}(\mathbf{w}, \Xi)(\delta \mathbf{w}, \delta \Xi)-\left(-i \partial_{x}\right)^{m} T_{n, \delta \xi_{n}, \delta \zeta_{n}}\right)\right\|_{\mathbf{G}_{3}} \\
& \quad \leqq \eta^{-3 / 2} \mu^{-1 / 2} \eta^{2-2 \rho_{1}+\rho_{1}|n|}\|\delta \mathbf{w}\|_{\mathbf{F}_{1}} \eta^{-\rho_{1}|n|} \\
& \quad \leqq \eta^{1 / 2-2 \rho_{1}} \frac{1}{n^{3}}\|\delta \mathbf{w}\|_{\mathbf{F}_{1}} .
\end{aligned}
$$

Note next that $T$ is an injection of $\mathbf{F}_{2}$ into $\mathbf{G}_{2}=\mathbf{F}_{1}$ as can be seen from

$$
\left\|\left\{T_{n, \delta \xi_{n}, \delta \zeta_{n}}\right\}_{n}\right\|_{\mathbf{G}_{2}}=\left\|\left\{T_{n, \delta \xi_{n}, \delta \zeta_{n}}\right\}_{n}\right\|_{\mathbf{F}_{1}}=\|\delta \Xi\|_{\mathbf{F}_{2}} .
$$

Similarly, we have

$$
\left(\left(-i \partial_{x}\right)^{m} T_{n, \delta \xi_{n}, \delta \zeta_{n}}\right)(X)=\delta \xi_{n} q_{n, 1}^{m}+\delta \zeta_{n} q_{n, 2}^{m},
$$

as is seen immediately from the definition (11.5). By (7.44), we have, on the "sector" $n$,

$$
P^{s}\left\{\left(-i \partial_{x}\right)^{m} T_{n, \delta \xi_{n}, \delta \zeta_{n}}\right\}(X)=0
$$


and

$$
P^{u}\left\{\left(-i \partial_{x}\right)^{m} T_{n, \delta \xi_{n}, \delta \zeta_{n}}\right\}(X)=\left(\delta \dot{\xi}_{n}, \delta \zeta_{n}\right)
$$

It remains to study the case $n=1$. We have to consider

$$
\begin{aligned}
& \delta \mathbf{T}_{1}^{(m)}(\mathbf{w}, \boldsymbol{\Xi})(\delta \mathbf{w}, \delta \Xi) \\
& \quad=S_{1}\left(-i \partial_{x}\right)^{m}\left(-\left(1-\mathbf{M}^{-1} \mathbf{Q}\right) \delta \mathbf{S}_{1}^{(2)}(\mathbf{w}, \Xi)(\delta \mathbf{w}, \Xi)(\delta \mathbf{w}, \delta \Xi)+\delta\left(\begin{array}{l}
a \\
b
\end{array}\right)\right)(\mathbf{X}) .
\end{aligned}
$$

Note that

$$
\begin{aligned}
\delta \mathbf{S}_{1}^{(2)}(\mathbf{w}, \Xi)(\delta \mathbf{w}, \delta \Xi)= & \left(1+\mathbf{M}^{-1} \mathbf{Q}\right) \delta\left(\begin{array}{l}
a \\
b
\end{array}\right)-\mathbf{M}^{-1} L^{-1}\left(\begin{array}{c}
\operatorname{Re} \delta h_{1}(\mathbf{w})+\delta \mathbf{K}_{12}(\mathbf{w}) \\
\operatorname{Im} \delta h_{1}(\mathbf{w})+\delta K_{22}(\mathbf{w})
\end{array}\right) \delta \mathbf{w} \\
& +\mathbf{M}^{-1}\left(\begin{array}{l}
\delta \mathbf{O}_{1}(\delta \mathbf{w}) \\
\delta \mathbf{O}_{2}(\delta \mathbf{w})
\end{array}\right)-\mathbf{M}^{-1} \mathbf{Q} \delta\left(\begin{array}{l}
a \\
b
\end{array}\right)+\mathbf{M}^{-1} T_{\delta \tau}+T_{1, \delta \xi_{1}, \delta \zeta_{1}} .
\end{aligned}
$$

Substituting into the above equation, and using Proposition 9.6, and (13.6) we get

$$
\begin{aligned}
& \| P_{1}^{(4)}\left(\delta \mathbf{T}_{1}(\mathbf{w}, \Xi)(\delta \mathbf{w}, \delta \Xi)-\left|\left(-i \partial_{x}\right)^{m}\left(1-\mathbf{M}^{-1} \mathbf{Q}\right)\left(\mathbf{M}^{-1} T_{\delta \tau}+T_{1, \delta \xi_{1}, \delta \zeta_{1}}\right)\right\rangle\right) \|_{\mathbf{G}_{3}} \\
& \quad \leqq \eta^{-1 / 2} \eta^{1} \eta^{-\rho_{1}}\|\delta \mathbf{w}\|_{\mathbf{F}_{1}} \leqq \eta^{1 / 2-\rho_{1}}\|\delta \mathbf{w}\|_{\mathbf{F}_{1}} .
\end{aligned}
$$

Note that by (9.63) and (11.28), we find

$$
\left.\| P_{1}^{(4)}\left|\left(-i \partial_{x}\right)^{m} \mathbf{M}^{-1} \mathbf{Q}\left(\mathbf{M}^{-1} T_{\delta \tau}+T_{1, \delta \xi_{1}, \delta \zeta_{1}}\right)\right\rangle\right)\left\|_{\mathbf{G}_{3}} \leqq \mathbf{O}\left(\eta^{1 / 2}\right)\right\| \delta \Xi \|_{\mathbf{F}_{1}} .
$$

Finally, it is immediate to see from (11.22) that

$$
\delta \mathbf{S}^{(1)}(\mathbf{w}, \Xi)(\delta \mathbf{w}, \delta \Xi)=S_{1}(\delta a(0)+i \delta b(0)) .
$$

\begin{tabular}{|c|c|c|c|c|c|}
\hline & $\tau$ & 1 & $n$ & $\xi_{1}, \zeta_{1}$ & $\xi_{n}, \zeta_{n}$ \\
\hline $\mathbf{G}_{1}$ & 0 & $\delta a(0)+i \delta b(0)$ & 0 & 0 & 0 \\
\hline $\mathbf{G}_{2}(1)$ & $\mathbf{M}^{-1} T_{\delta \tau}$ & $\begin{array}{ll}\delta a & 0 \\
0 & \delta b\end{array}$ & 0 & $T_{1, \delta \xi_{1}, \delta \zeta_{1}}$ & 0 \\
\hline $\mathbf{G}_{2}(n)$ & 0 & 0 & $\delta w_{n}$ & 0 & $T_{n, \delta \xi_{n}, \delta \zeta_{n}}$ \\
\hline $\mathbf{G}_{3}(1)$ & 0 & 0 & 0 & $P^{u}\left\{\left(-i \partial_{x}\right)^{k} T_{1, \delta \xi_{1}, \delta \zeta_{1}}\right\}(X)$ & 0 \\
\hline $\mathbf{G}_{3}(n)$ & 0 & 0 & 0 & 0 & $P^{u}\left\{\left(-i \partial_{x}\right)^{k} T_{n, \delta \xi_{n}, \delta \xi_{n}}\right\}(X)$ \\
\hline
\end{tabular}

Summarizing, we have shown that $\delta \mathbf{S}$ is, up to smaller terms, a "matrix" of the following form:

We label the columns by " 1 ", $\tau$, " $n$ ", and $\xi_{n}, \zeta_{n}$, denoting thus in short the subspaces of $\mathbf{F}_{1}$ ("1" and " $n$ "), respectively of $\mathbf{F}_{2}$. Similarly the rows will be denoted $\mathbf{G}_{1}(=\mathbb{C})$, $\mathbf{G}_{2}$ ("1" and " $n$ " as above) and $\mathbf{G}_{3}(n)$, respectively $\mathbf{G}_{3}(1)$ (for the components of $\mathbf{G}_{3}$ ). Then we see that $\delta \mathbf{S}$ is, up to terms which vanish like some power of $\eta$, of the form

Note that we have written the matrix elements as functions, rather than in the conventional way. As an example, we should write, instead of $T_{n, \delta \xi_{n}, \delta \zeta_{n}}$, cf. (11.5), only $T_{n}$, and define

$$
\begin{gathered}
T_{n}: \mathbb{R}^{2} \rightarrow \mathbf{G}_{2}(n), \\
T_{n}(u, v)=\left(\begin{array}{c}
u \operatorname{Re} e^{i q_{n, 1}(x-X)}+v \operatorname{Re} e^{i q_{n, 2}(x-X)} \\
u \operatorname{Im} e^{i q_{n, 1}(x-X)}+v \operatorname{Im} e^{i q_{n, 2}(x-X)}
\end{array}\right) .
\end{gathered}
$$


We have preferred the more symbolic notation for its legibility.

We want to argue now that the matrix in (13.16) is invertible. This would be obvious if it were upper triangular. It is not, but the following simple change of coordinates in function space will bring it to triangular form. Instead of the variables $(a, b)$ and $\tau$, we consider the variables

$$
\left(\begin{array}{l}
a^{\prime} \\
b^{\prime}
\end{array}\right)=\left(\begin{array}{l}
a \\
b
\end{array}\right)+\mathbf{M}^{-1} T_{\tau}=\left(\begin{array}{l}
a \\
b
\end{array}\right)+\tau \mathbf{M}^{-1}\left(\begin{array}{c}
l^{\prime} / l(0) \\
0
\end{array}\right),
$$

and $\tau$. It is obvious that this brings the matrix to upper triangular form. Note also that this change of coordinates is invertible, and by the methods leading to (9.53), we see that from the differentiability of $\left(l^{\prime}, 0\right)$ one has

$$
\left\|\mathbf{M}^{-1} T_{\tau=1}\right\|_{\mathbf{H}_{\alpha, X}}=\mathbf{O}(1) .
$$

Here, we define the inverse of $\mathbf{M}$ slightly differently from Eq. (9.50) by replacing the upper limit $X$ of integration by $+\infty$. The integrals exist and lead to (13.19) as can be easily checked. Hence (13.16) is invertible, with inverse bounded by $\mathbf{O}(1)$ uniformly in $\eta$. It follows that $\delta \mathbf{S}$ is invertible, with inverse bounded uniformly in $\eta$. Hence the operator $\mathbf{S}$ has a fixed point in the ball defined by (12.1), when $\eta$ is sufficiently small and this fixed point is a solution of the existence problem for the fronts. The Main Theorem is proven.

Acknowledgements. We thank J. Langer for introducing us to the problem and for freely sharing with us his insights. This work was started in 1982, and we have profited thereafter from the hospitality and the financial support of several institutions and organizations which made our collaboration possible: ITP Santa Barbara, IMA Minneapolis, IHES Bures-sur-Yvette, Rutgers University (NSF Grant DMR8114726), CNRS, Fonds National Suisse.

\section{References}

1. Aronson, D. G., Weinberger, H.: Multidimensional nonlinear diffusions arising in population genetics. Adv. Math. 30, 30 (1978)

2. Langer, J. S.: Instabilities and pattern formation in crystal growth. Rev. Mod. Phys. 52, 1 (1980)

3. Dee, G., Langer, J. S.: Propagating pattern selection. Phys. Rev. Lett. 50, 383 (1983)

4. Langer, J. S., Müller-Krumbhaar, H.: Mode selection in a dendrite-like nonlinear system. Phys. Rev. A27, 499 (1982)

5. Hirsch, M. W., Pugh, C. C., Shub, M.: Invariant manifolds. Lecture Notes in Mathematics, Vol. 583. Berlin, Heidelberg, New York: Springer

6. Yosida, K.: Functional analysis. Berlin, Heidelberg, New York: Springer 1971

7. Bramson, M.: Convergence of solutions of the Kolmogoroff equations to traveling waves. Mem. Am. Math. Soc. 285 (1983)

Communicated by A. Jaffe

Received April 1, 1986 